This is the peer reviewed accepted manuscript of:

Chen B, Dragomir MP, Fabris L, Bayraktar R, Knutsen E, Liu X, Tang C, Li Y, Shimura T, Ivkovic TC, De los Santos MC, Anfossi S, Shimizu M, Shah MY, Ling H, Shen P, Multani AS, Pardini B, Burks JK, Katayama H, Reineke LC, Huo L, Syed M, Song S, Ferracin M, Oki E, Fromm B, Ivan C, Bhuvaneshwar K, Gusev Y, Mimori K, Menter D, Sen S, Matsuyama $T$, Uetake $H$, Vasilescu $C$, Kopetz $S$, Parker-Thornburg J, Taguchi A, Hanash SM, Girnita L, Slaby O, Goel A, Varani G, Gagea M, Li C, Ajani JA, Calin GA, The Long Noncoding RNA CCAT2 induces chromosomal instability through BOP1 AURKB signaling, Gastroenterology (2020),

Final version available at: https://doi.org/10.1053/i.gastro.2020.08.01

Rights / License:

The terms and conditions for the reuse of this version of the manuscript are specified in the publishing policy. For all terms of use and more information see the publisher's website.

This item was downloaded from IRIS Università di Bologna (https://cris.unibo.it/)

When citing, please refer to the published version. 


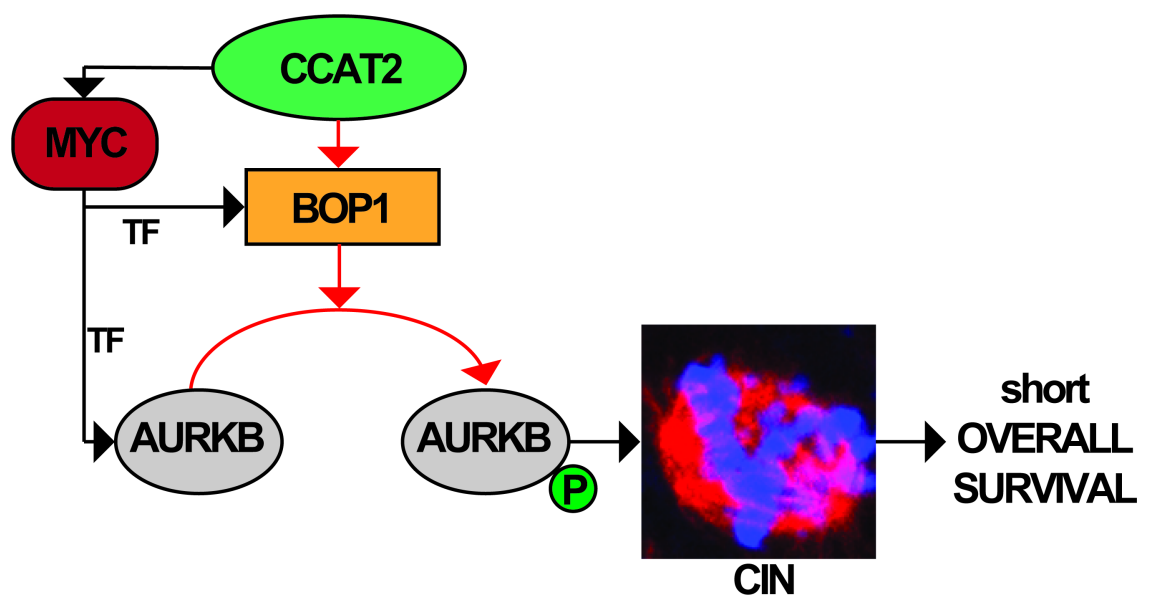




\title{
The Long Noncoding RNA CCAT2 induces chromosomal instability through BOP1 - AURKB signaling
}

\begin{abstract}
Baoqing Chen ${ }^{1,2,29}{ }^{*}$, Mihnea P. Dragomir ${ }^{1,3}{ }^{*}$, Linda Fabris $^{1}{ }^{*}, \operatorname{Recep~Bayraktar}^{1}{ }^{*}$, Erik Knutsen ${ }^{1,4}, \mathrm{Xu}$ Liu $^{1,5}$, Changyan Tang 6 , Yongfeng Li ${ }^{1}$, Tadanobu Shimura ${ }^{7}$, Tina Catela Ivkovic ${ }^{8}$, Mireia Cruz De los Santos ${ }^{1}$, Simone Anfossi ${ }^{1}$, Masayoshi Shimizu', Maitri Y. Shah ${ }^{1}$, Hui Ling ${ }^{1}$, Peng Shen ${ }^{1,9}$, Asha S. Multani10, Barbara Pardini1,30, 31, Jared K. Burks ${ }^{11}$, Hiroyuki Katayama12, Lucas C. Reineke ${ }^{13}$, Longfei Huo ${ }^{14}$, Muddassir Syed ${ }^{14}$, Shumei Song ${ }^{14}$, Manuela Ferracin ${ }^{15}$, Eiji Oki ${ }^{16}$, Bastian Fromm 17, 18, Cristina Ivan 1, 19, Krithika Bhuvaneshwar 20, Yuriy Gusev20, Koshi Mimori21, David Menter ${ }^{14}$, Subrata Sen 22, Takatoshi Matsuyama ${ }^{23}$, Hiroyuki Uetake 24, Catalin Vasilescu3, 25, Scott Kopetz ${ }^{14}$, Jan Parker-Thornburg ${ }^{10}$, Ayumu Taguchi22, Samir M. Hanash ${ }^{12}$, Leonard Girnita ${ }^{26}$, Ondrej Slaby', 27, Ajay Goel7, 32, Gabriele Varani6, Mihai Gagea ${ }^{28}$, Chunlai Li 1, \&, Jaffer A. Ajani14, \&, George A. Calin 1, 19, 33, \&
\end{abstract}

* - author names in bold designate shared co-first authorship.

\& - co-corresponding authors;

${ }^{1}$ Department of Experimental Therapeutics, The University of Texas MD Anderson Cancer Center, Houston, TX 77030, USA.

2 Department of Radiation Oncology, State Key Laboratory of Oncology in South China, Collaborative Innovation Center for Cancer Medicine, Sun Yat-sen University Cancer Center, Guangzhou, Guangdong, China;

3 Department of General Surgery, Fundeni Clinical Hospital, Carol Davila University of Medicine and Pharmacy, Bucharest, Romania.

${ }^{4}$ Department of Medical Biology, Faculty of Health Sciences, UiT - The Arctic University of Norway, Tromsø, Norway.

${ }^{5}$ Department of Thoracic Surgery, The First Affiliated Hospital of Xi'an Jiaotong University, Xi'an, China

${ }^{6}$ Department of Chemistry, University of Washington, Seattle, WA 98195, USA.

${ }^{7}$ Center for Gastrointestinal Research; Center for Translational Genomics and Oncology, Baylor Scott \& White Research Institute, Charles A Sammons Cancer Center, Baylor University Medical Center, Dallas, USA.

${ }^{8}$ Central European Institute of Technology, Masaryk University, Brno, Czech Republic

${ }^{9}$ Department of Oncology, Nanfang Hospital, Southern Medical University, Guangzhou, China.

10 Department of Genetics, The University of Texas MD Anderson Cancer Center, Houston, TX, USA.

${ }^{11}$ Department of Leukemia, The University of Texas MD Anderson Cancer Center, Houston, TX, USA. 
${ }^{12}$ Department of Clinical Cancer Prevention, University of Texas MD Anderson Cancer Center, Houston, TX, USA.

${ }^{13}$ Department of Neuroscience, Baylor College of Medicine, Houston, TX, USA.

${ }^{14}$ Department of Gastrointestinal Medical Oncology, The University of Texas MD Anderson Cancer Center, Houston, TX, USA.

15 Department of Experimental, Diagnostic and Specialty Medicine (DIMES), University of Bologna, 40126 Bologna, Italy.

16 Department of Surgery and Science, Graduate School of Medical Sciences, Kyushu University, Fukuoka, Japan.

17 Science for Life Laboratory, Department of Molecular Biosciences, The Wenner-Gren Institute, Stockholm University, Stockholm, Sweden

18 Department of Tumor Biology, Institute for Cancer Research, The Norwegian Radium Hospital, Oslo University Hospital, Oslo, Norway

${ }^{19}$ Center for RNA Interference and Non-coding RNAs, The University of Texas MD Anderson Cancer Center, Houston, TX 77030, USA.

20 Innovation Center for Biomedical Informatics, Georgetown University, Washington, DC, USA

${ }^{21}$ Department of Surgery, Kyushu University Beppu Hospital, Beppu, Japan.

${ }^{22}$ Department of Translational Molecular Pathology, The University of Texas MD Anderson Cancer Center, Houston, TX, 77030, USA.

${ }^{23}$ Department of Gastrointestinal Surgery, Tokyo Medical and Dental University Graduate School of Medicine, Tokyo, Japan.

${ }^{24}$ Department of Specialized Surgeries, Graduate School, Tokyo Medical and Dental University, Tokyo, Japan.

25 "Carol Davila University of Medicine and Pharmacy", Bucharest, Romania

${ }^{26}$ Department of Oncology-Pathology, Bioclinicum, Karolinska Institute and Karolinska University Hospital, SE-171 647 Stockholm, Sweden.

27 Department of Biology, Faculty of Medicine, Masaryk University, Brno, Czech Republic

${ }^{28}$ Department of Veterinary Medicine and Surgery, The University of Texas MD Anderson Cancer Center, Houston Texas 77030, USA.

${ }^{29}$ Department of Thoracic Oncology, Cancer Center and State Key Laboratory of Biotherapy, West China Hospital, Sichuan University, Chengdu, Sichuan, China.

${ }^{30}$ present address: Italian Institute for Genomic Medicine (IIGM), Candiolo, Italy.

${ }^{31}$ present address: Candiolo Cancer Institute, FPO-IRCCS, Candiolo, Italy.

32 present address: Department of Molecular Diagnostics and Experimental Therapeutics, Beckman Research Institute of City of Hope Comprehensive Cancer Center, Duarte, CA 
${ }^{33}$ Lead Contact.

Running title: CCAT2 induces chromosomal instability via BOP1

Grant Support: Dr. Calin is the Felix L. Haas Endowed Professor in Basic Science. Work in Dr. Calin's laboratory is supported by National Institutes of Health (NIH/NCATS) grant UH3TR00943-01 through the NIH Common Fund, Office of Strategic Coordination (OSC), the NCI grants 1R01 CA182905-01 and 1R01CA222007-01A1, an NIGMS 1R01GM122775-01 grant, a U54 grant \#CA096297/CA096300 UPR/MDACC Partnership for Excellence in Cancer Research 2016 Pilot Project, a Team DOD (CA160445P1) grant, a Chronic Lymphocytic Leukemia Moonshot Flagship project, the UT MD Anderson Cancer Center Duncan Family Institute for Cancer Prevention and Risk Assessment, a Sister Institution Network Fund (SINF) 2017 grant, and the Estate of C. G. Johnson, Jr. Dr. Pardini is recipient of a Fulbright Research Scholarships (year 2018). Work at the University of Washington was supported by NIGMS R35 GM121487. The work of Dr. Baoqing Chen is supported by National Natural Science Foundation of China (No. 81902462). The work in Dr. Goel's lab is supported by the grants CA72851, CA181572 and CA202797 from the National Cancer Institute, National Institute of Health. The work of Dr. Parker-Thornburg was supported by the grants 5R50CA211121-03 and CCSG for the GEMF core.

Abbreviations: CIN, chromosomal instability; ncRNA, non-coding RNA; lncRNA, long non-coding RNA; CCAT2, colon cancer associated transcript 2 gene; CRC, colorectal cancer; BOP1, BOP1 ribosomal biogenesis factor; AURKB, aurora kinase B; MSS, microsatellite stable; MSI microsatellite instable; MDS, myelodysplastic syndrome; GC, gastric cancer; DSB, double strand breaks; AOM, azoxymethane; DSS, dextran sulphate sodium; IPA, Ingenuity Pathway Analysis; GEA, gene expression analysis; MS, mass spectrometry; RIP, RNA immunoprecipitation; NLS, nuclear localization signal; CHX, cycloheximide; TF, transcription factor; AURKA, aurora kinase A; pAURKB, phosphorylated aurora B; PDX, patient derived xenograft; PDO, patient derived organoid; OS, overall survival; RFS, recurrence free survival.

Correspondence: George A. Calin, M.D., Ph.D. Professor, Department of Experimental Therapeutics, Center for RNA Interference and Non-Coding RNAs, Department of Experimental Therapeutics - Unit 1950, The University of Texas MD Anderson Cancer Center, P.O. Box 301429, Houston, Texas 77030-1429, gcalin@mdanderson.org,or to Jaffer Ajani, M.D., at jajani@mdanderson.org Department of Gastrointestinal Medical Oncology, The University of Texas MD Anderson Cancer Center, Houston, TX, USA, or to Chunlai Li, Ph.D. at CLi10@mdanderson.org, Department of Experimental Therapeutics, The University of Texas MD Anderson Cancer Center, Houston, TX, USA.

Disclosure: The authors declare no competing interests. 
Transcript profiling: Gene expression Omnibus accession number from data used in this study (GSE106581, GSE133590).

Author contributions: Conceptualization B.C., M.P.D., and G.A.C.; Methodology, B.C, M.P.D., C.L., E.K., X.L., C.T., T.S., T.C.I, M.Y.S., H.L., P.S., A.S.M., B.P., J.K.B., H.K., L.C.R., Y.G., D.M., S.Sen, J.P.T., M.G., A.T., L.G. G.A.C.; Formal Analysis, B.C., M.P.D., E.K., C.T., T.S., T.C.I, A.S.M., L.C.R., M.F., B.F., C.I., K.B., Y.G., Investigation, B.C., M.P.D., C.L., L.F., R.B., E.K., Y.L., C.T., X.L., T.S., T.C.I., S.A., M.Shimizu, M.C.S., A.S.M., B.P., H.K., L.C.R., L.H., M.Syed, S.Song, M.F., E.O., T.M., H.U., A.T., Writing - Original Draft, B.C., M.P.D., and G.A.C.; Writing - Review \& Editing, all authors.; Supervision, C.V., S.K., S.M.H., L.G., K.M., A.G., O.S., G.V., J.A.A., and G.A.C.; Funding Acquisition, J.A.A. and G.A.C..

Acknowledgements: We thank the MDACC Cytogenetics and Cell Authentication Core for technical assistance in the cytogenetic analysis. 


\begin{abstract}
Background \& Aims: Chromosomal instability (CIN) is a carcinogenesis event that promotes metastasis and resistance to therapy, by unclear mechanisms. Expression of the colon cancer associated transcript 2 gene (CCAT2), which encodes a long noncoding RNA (lncRNA), associates with CIN, but little is known about how CCAT2 IncRNA regulates this cancer enabling characteristic.
\end{abstract}

Methods: We performed cytogenetic analysis of colorectal cancer (CRC) cell lines (HCT116, KM12C/SM, and HT29) overexpressing CCAT2 and colon organoids from C57BL/6N mice with the CCAT2 transgene and without (controls). CRC cells were also analyzed by immunofluorescence microscopy, $\gamma$-H2AX, and senescence assays. CCAT2 transgene and control mice were given azoxymethane and dextran sulphate sodium (DSS) to induce colon tumors. We performed gene expression array and mass spectrometry to detect downstream targets of CCAT2 IncRNA. We characterized interactions between CCAT2 with downstream proteins using MS2 pulldown, RNA immunoprecipitation, and SHAPE analyses. Downstream proteins were overexpressed in CRC cells and analyzed for CIN. Gene expression levels were measured in CRC and non-tumor tissues from 5 cohorts, comprising more than 900 patients.

Results: High expression of CCAT2 induced CIN in CRC cell lines and increased resistance to 5fluorouracil and oxaliplatin. Mice that expressed the CCAT2 transgene developed chromosome abnormalities, and colon organoids derived from crypt cells of these mice had a higher percentage of chromosome abnormalities compared to organoids from control mice. The transgenic mice given azoxymethane and DSS developed more and larger colon polyps than control mice given these agents. Microarray analysis and mass spectrometry indicated that expression of CCAT2 increased expression of genes involved in ribosome biogenesis and protein synthesis. CCAT2 lncRNA interacted directly with and stabilized BOP1 ribosomal biogenesis factor (BOP1). CCAT2 also increased expression of MYC, which activated expression of BOP1. Overexpression of BOP1 in CRC cell lines resulted in chromosomal missegregation errors, and increased colony formation, and invasiveness, whereas BOP1 knockdown reduced viability. BOP1 promoted CIN by increasing the active form of aurora kinase B (AURKB), which regulates chromosomal segregation. BOP1 was overexpressed in polyp tissues from CCAT2 transgenic mice, compared to healthy tissue. CCAT2 lncRNA and BOP1 mRNA or protein were all increased in microsatellite stable tumors (characterized by CIN), but not in tumors with microsatellite instability, compared with non- 
tumor tissues. Increased levels of CCAT2 IncRNA and BOP1 mRNA correlated with each other and with shorter survival times of patients.

Conclusions: We found that overexpression of CCAT2 in colon cells promotes CIN and carcinogenesis, by stabilizing and inducing expression of BOP1 an activator of AURKB. Strategies to target this pathway might be developed for treatment of patients with microsatellite stable colorectal tumors.

KEY WORDS: MSS, aneuploidy, tumorigenesis, non-coding RNA 


\section{Introduction}

Considered one of the initial molecular events and one of the most noticeable pathogenic feature of cancer, genomic instability ${ }^{1}$, was predicted by Theodore Boveri, over 100 years ago ${ }^{2}$. Chromosomal instability (CIN) is one of the most common forms of genomic instability, and is characterized by high rates of structural and numerical chromosomal aberrations ${ }^{3}$. By constantly making new aneuploid genomes, CIN causes tumor cell heterogeneity, upon which clonal selection can act 4 .

One of the main causes of CIN is the asymmetrical segregation of chromosomes during the metaphase as a consequence of abnormal spindle formation 5 that will also induce anaphase bridges during mitosis, which is a key feature of CIN ${ }^{6}$. CIN is associated with poor survival, metastases, and therapy resistance in cancer 7. Yet, the complex molecular mechanism(s) underlying the process remains unclear. Tens of protein coding genes and only few non-coding RNAs (ncRNAs) have been associated with the occurrence of CIN ${ }^{8}$. Nonetheless, all these genes are altered in a small fraction of patients and their involvement in CIN has been studied mainly in in vitro systems.

The long-non-coding RNA (lncRNA) colon cancer associated transcript 2 (CCAT2), is upregulated in microsatellite stable (MSS) colorectal cancers (CRC), tumors characterized by CIN and in which CCAT2 promotes metastases 9 and influence glutamine metabolism ${ }^{10 .}$ Additionally, CCAT2 IncRNA was also reported to be involved in the initiation of myelodysplastic syndrome (MDS) ${ }^{11}$, a premalignant condition associated with abnormal chromosomes ${ }^{12}$. These findings imply that CCAT2 plays a role in early stages of carcinogenesis. Despite its proven clinical value, the exact molecular mechanisms by which CCAT2 IncRNA induces CIN are unexplored. Here, we have analyzed, for the first time, the molecular pathways through which a IncRNA induces CIN both in vitro and in vivo, and we identify CCAT2 as a regulator of MYC-BOP1-AURKB pathway causing CIN.

\section{Materials and Methods}

\section{Patient sample collection}

For this study we used five different patient cohorts (Supplementary Tables 1-4): Cohort A (TCGA colorectal cancer cohort) was used for screening of the PES1, BOP1, and WDR12 (PeBoW) complex expression in MSS versus MSI subtypes of CRC; Cohort B for validating CCAT2 IncRNA and 
PeBoW complex mRNA overexpression in CRC; Cohort $\mathbf{C}$ for validating CCAT2 lncRNA and BOP1 mRNA role in MSS CRC subtype; Cohort D for further validation and prognosis analysis of CCAT2 lncRNA and PeBoW complex mRNA expression in CRC; and Cohort E (described previously in ${ }^{10}$ ) for validation of the mechanism at the protein level. Written informed consent was obtained from each patient, and the study was approved by the institutional review boards of all the involved institutions.

Additional methods are available in Supplementary material and methods. Primers and antibody information are available in Supplementary Tables 5 and 6.

\section{RESULTS}

\section{CCAT2 IncRNA induces CIN and activates pathways associated with ribosomal proteins}

We decided to investigate the causal relationship between CCAT2 and CIN in cancer because of multiple lines of evidence. First, in multiple CRC and gastric cancer (GC) cell lines used, we observed that an aberrant chromosomal number is associated with high levels of CCAT2 lncRNA (Supplementary Figure 1A and Supplementary Table 7). Second, cytogenetic analysis suggested that HCT116 clones with exogenous CCAT2 over-expression (named here HCT116 ${ }^{\text {CCAT2) }}$ have a higher percentage of chromosome abnormalities (including breaks, fusion, and polyploidy) compared to HCT116 cells transduced with an empty vector (HCT116 ${ }^{\text {Empty }}$ ) (31.0\% versus 11.9\%, $P=.001$ ). Similar results were found in the KM12SM cells (highly metastatic) compared to KM12C (poorly metastatic, from same patient) (44.5\% versus 33.3\%, $P=.1099$ ) (Figure 1A and 1B) KM12SM, with two times higher CCAT2 levels as KM12C, presents also higher degree of CIN (Supplementary Figure 1A and Supplementary Table 7). Third, by double strand breaks (DSB) analysis using $\gamma$-H2AX, the number of cells with DSBs was higher in HCT116CCAT2 (39/152, 25.7\%) compared to HCT116 Empty $(2 / 162,1.2 \%)(P<.0001)$ (Supplementary Figure 1B). Next, when we treated the cells with the DNA strand breaks inducer bleomycin, the number of DSBs was 42/126 in HCT116 ${ }^{\text {CCAT2 }}$ cells $(33.3 \%)$ and $28 / 138$ in HCT116 Empty cells $(20.3 \%)(P=.0165)$ (Supplementary Figure 1C). Additionally, we observed that HCT116 CCAT2 showed significantly lower chemosensitivity to 5-Flurouracil $(P=.003, P=.006$ and $P=.0001$, respectively) (Supplementary Figure 1D) and to oxaliplatin $(P<.0001$ for all three concentrations) (Supplementary Figure 1E) compared to HCT116 Empty. One of the possible mechanisms through which cells tolerate DNA damage and stop CIN, is senescence ${ }^{13}$. We observed that HCT116CCAT2 
clones had ten times lower number of senescent cells compared to HCT116 $6^{\text {Empty }}(P=.0001)$ (Supplementary Figure 1F). Fourth, HCT116 ${ }^{\text {CCAT2 }}$ clones had a significant increase in abnormal spindles, along with a higher percentage of anaphase bridges, indirect indicators of CIN 14, compared with HCT116 Empty (mean $13.9 \%$ versus $6.2 \%, P=.009$ and $51.8 \%$ versus $24.7 \%, P$ $=.0052$, respectively) (Figure 1C and 1D).

Fifth, our recently developed mouse model 11 indicates that CCAT2 IncRNA can trigger CIN in vivo (Supplementary Figure 1G). We found that the karyotypes from the bone marrow tissue of CCAT2 mice showed more chromosomal abnormalities compared to WT littermates (Supplementary Figure 1H). Finally, we developed normal colon organoid cultures from the crypt cells of CCAT2 and WT mice. The CCAT2 IncRNA expression remained significantly higher in CCAT2 organoids compared to WT organoids (Supplementary Figure 1I). No important morphology differences were observed between CCAT2 and WT mice derived organoids (Supplementary Figure 1J), but a more rapid growth rate was measured for the CCAT2 organoids $(P=.001)$ (Supplementary Figure 1K). After 3 passages we performed cytogenetic analysis, which showed that normal colon organoids from CCAT2 mice had a significantly higher percentage of chromosome abnormalities compared to organoids from WT mice $(68.6 \%$ versus $51.4 \%, P$ $=.0094$ ) (Figure 1E and 1F), denoting that CIN induced by CCAT2 preceded tumor development.

To find out if CCAT2 IncRNA plays a role in the acceleration and progression of colon tumors we used the azoxymethane/dextran sulphate sodium (AOM/DSS) model, which was previously reported to induce CIN ${ }^{15}$. CCAT2 transgenic and WT mice were injected with AOM and then subjected to four rounds of DSS (Figure 1G). Macroscopic analysis revealed that CCAT2 mice had significantly higher number of polyps $(P=.0143)$, the surface of colon mucosa covered by polyps was significantly larger $(P=.0088)$, and the average polyp diameter was significantly longer $(P=$ .0009) than WT mice (Figure 1H and 1I). Next, we performed Hematoxylin and Eosin (H\&E) staining and histopathological evaluation of the colon of WT and CCAT2 mice (Figure 1J). The size of the largest polyp was significantly greater in CCAT2 mice compared to WT mice $(P=.0093)$ (Figure 1K). Importantly in CCAT2 mice the grade of hyperplasia of the colon glands was higher in comparison with WT mice (Grade 3-4: CCAT2 - 71.4\% versus WT - 50.0\%, $P=.0024$ ) (Figure 1L) and the mice had a higher incidence of dysplastic colon glands in comparison with the control group (71.4\% versus 50.0\%, $P=.0024$ ) (Figure 1M).

We used microarray gene expression analysis (GEA) to identify downstream targets of CCAT2 
on BM cells obtained from WT mice and CCAT2 transgenic mice (GSE106581). Ingenuity Pathway Analysis (IPA) revealed that EIF2, mTOR, and regulation of eIF4 and p70S6K pathways were all significantly up-regulated in the CCAT2 mouse model when compared to WT mice (Figure 1N). All these pathways are related to protein synthesis ${ }^{16}$. Among the common genes involved in these pathways, those related to ribosomal proteins and ribosome biogenesis were enriched (Figure $\mathbf{1 N}$ ). The deregulation of proteins involved in ribosome biogenesis or ribosomal proteins have been previously linked to CIN ${ }^{17}$.

\section{CCAT2 IncRNA interacts with BOP1}

Considering that the lncRNA CCAT2 is localized mostly in the nucleus (Supplementary Figure 2A), we performed Mass Spectrometry (MS) on HCT116 cells transiently transfected with CCAT2MS2 vectors, and isolated CCAT2 IncRNA interacting nuclear proteins. Again, we found an enrichment in proteins involved in protein translation and ribosomal biogenesis (Supplementary Table 8). By screening the literature, we found that, one of these candidate proteins, BOP1 ribosomal biogenesis factor (BOP1), was previously reported to affect spindle formation and cause CIN in CRC 18,19, therefore, we selected it for further analysis. BOP1 and CCAT2 genes are both located on chromosome 8q24, a region amplified in many cancers ${ }^{20}$. BOP1 protein is one of the three components, which includes also PES1 and WDR12 proteins, of the PeBoW complex, a regulator of rRNA processing affecting ribosome biogenesis ${ }^{21}$.

To explore the interaction between CCAT2 lncRNA and BOP1 protein, an in vivo MS2-pull down assay was conducted in COLO320 cells. BOP1 was retrieved through the CCAT2-MS2 construct and not by MS2-empty vector, while WDR12 and PES1 of the PeBoW complex, were only minimally retrieved (Figure 2A). To validate these results, RNA immunoprecipitation (RIP) using BOP1, WDR12, and PES1 antibodies was performed. CCAT2 was identified $\sim 5$ and $\sim 11$ times higher in the BOP1 immunoprecipitate than PES1 and WDR12 precipitates, respectively (Figure 2B and Supplementary Figure 2B). This suggests that BOP1 protein is a strong interactor of CCAT2 lncRNA, and the WDR12 and PES1 signals are probably identified due to indirect, low stability interactions with BOP1 protein. In vitro pull-down, was consistent with the previous results: the biotin-labeled CCAT2 IncRNA, but not biotin-labeled controls, pulled down the recombinant BOP1 (Figure 2C). 
The BOP1 protein consists of seven WD40 repeats (from amino acids 411 to 746, Supplementary Figure 2C), which are discreet domains that interact with proteins and RNAs, providing platforms to assemble functional complexes ${ }^{22}$. To characterize the region(s) of BOP1 that directly interacts with CCAT2 IncRNA, we generated a series of BOP1 truncations with deletion of each WD repeats (Figure 2D), and one with a deletion of the nuclear localization signal (NLS). Using RIP assays, we observed that the abundance of CCAT2 in WD 1, 2, 3, 5, 6, and 7 truncated BOP1 groups was decreased compared to other groups. This suggested that deletion of any individual WD repeats, except WD 4, abolishes the BOP1-CCAT2 interaction (Figure 2D).

In order to map the RNA sequence of CCAT2 that interacts with BOP1 protein, RNA pull-down was performed using a set of 10 CCAT2 segments. Results indicated that segment 3 (nucleotides 333 to 435 ) interacted directly with BOP1 protein (Figure 2E). Although the conservation of CCAT2 gene is high in mammals $(75.4 \% \pm 19.9, \mathrm{n}=66$ species) (Supplementary Figure 2D), the interacting segment 3 is mostly conserved in primates (more than $90 \%$ conservation), than in other mammals (30\% - 70\%) (Supplementary Figure 2E). The secondary structure of the CCAT2 IncRNA region that spans nucleotide 207 to 492, which includes segment 3, was determined experimentally by SHAPE. Consistent with the results from Figure 2E, some active regions were determined, suggesting that segment 3, provides a platform for protein interactions (Figure 2F and Supplementary Figure 2F and 2G).

\section{CCAT2 IncRNA up-regulates BOP1 in vitro and in vivo}

To investigate the effect of CCAT2 IncRNA on the PeBoW complex, we verified the expression of the PeBoW complex components in CCAT2 overexpression clones. In the cell lines with high levels of CCAT2, HCT116CCAT2, and KM12SM, BOP1 mRNA levels were four and two times higher while protein levels were eleven and three times higher compared to HCT116 Empty and KM12C (Figure 3A). In both HCT116 and KM12SM, 50-60\% knock-down of CCAT2 resulted in down regulation of BOP1 protein and mRNA levels (Figure 3B).

These results indicated that CCAT2 IncRNA mainly regulates BOP1 by a post-transcriptional mechanism. To test if CCAT2 IncRNA affects the stability of BOP1 protein, cyclohexamide (CHX) chase assay was conducted on KM12SM cells with transient CCAT2 over-expression. Four hours after CHX addition, BOP1 protein expression started decreasing more rapidly in the Empty clone than in the CCAT2 overexpressing clone (Figure 3C). We then checked the intracellular localization of BOP1 protein: as expected, in HCT116 ${ }^{\text {CCAT2 }}$ cells, BOP1 was enriched in the nuclear fraction 
compared to the HCT116 ${ }^{\text {Empty }}$ clone, while there was no difference in the cytoplasm (Figure 3D). These data were confirmed in DLD-1 ${ }^{\text {Empty }}$ and DLD-1 ${ }^{\text {CCAT2 }}$ (Supplementary Figure 3A).

Using the CCAT2 mouse model ${ }^{11}$, we observed that the stable overexpression of CCAT2 IncRNA in mice leads to the up-regulation of BOP1 mRNA and protein in healthy colon tissue of CCAT2 mice compared to WT mice, hence this phenomenon is preceding tumor formation (Figure 3E and Supplementary Figure 3B). Moreover, we detected a higher protein level of BOP1 in the bone marrow of CCAT2 mice, who we previously showed to develop MDS and display chromosomal abnormalities 11, compared to WT mice (Supplementary Figure 3C). We also checked BOP1 mRNA level in macroscopically unaffected colon tissues and in polyps from AOM/DSS treated CCAT2 transgenic mice. We observed that the mRNA level of BOP1 was further increased in polyps compared to normal colon in CCAT2 transgenic mice $(P=.0095)$ (Figure 3F).

In our previous study ${ }^{9}$, we reported that CCAT2 up-regulates MYC protein through TCF7L2. We found that MYC is also a predicted transcription factor (TF) for BOP1 gene (Supplementary Figure 3D and 3E). CHIP-Seq data (UCSC Genome Browser Assembly) showed that MYC binds to its specific sequence (CACGTG) located in the 5' region of BOP1 gene and acts as a TF for BOP1 23 (Supplementary Figure 3F). Next, we verified whether MYC could regulate the expression of BOP1 using a doxycycline inducible HCT116 MYC tet-on system. BOP1 mRNA levels were about 3 times higher $12 \mathrm{~h}$ after induction of MYC and remained stable until $24 \mathrm{~h}$. Accordingly, the protein level of BOP1 increased after 12h, and reached an even higher level $24 \mathrm{~h}$ after induction (Supplementary Figure 3G). In summary, CCAT2 IncRNA regulates BOP1 mainly at the protein level by direct binding, but also positively regulates its transcription through MYC.

\section{Overexpression of BOP1 promotes CIN}

To confirm the role of BOP1 in triggering CIN, we stably overexpressed BOP1 in HCT116, KM12SM, and HT29 cell lines (Supplementary Figure 4A and 4B) and performed cytogenetic analysis. After few passages (5-10), no difference on genomic instability was observed (Supplementary Figure 4C). However, after a longer propagation time ( $>15$ passages), B0P1 clones started to present chromosomal aberrations. HCT116 cells with BOP1 overexpression (HCT116 ${ }^{\mathrm{BOP} 1}$ ) showed a higher frequency of chromosomal abnormalities (fusions, breaks, and fragmentation) (Figure 4A panels (i), (ii), and (iii)) compared to HCT116 Empty (31.4\% versus 8.3\%, $P=.0001$ ) (Figure 4B). The KM12SM ${ }^{\mathrm{BOP} 1}$ clones showed a higher percentage of aberrant metaphases compared to control cells $(38.0 \%$ versus $22.0 \%, P=.0136)$. Specifically, a higher 
percentage of polyploidy or tetraploidy was observed in the KM12SM ${ }^{\mathrm{BOP} 1}$, indicative of a greater chromosome segregation failure (Figure 4A panel (iv) and Figure 4B). Moreover, a significantly higher genomic vulnerability was found in НТ29воР1 (28.6\% versus $2.9 \%, P<.0001$ ), including canaphases (Figure 4A panel (v) and Figure 4B).

BOP1 has previously been reported to alter the spindle apparatus and to cause aberrant lagging chromosomes ${ }^{18}$. As shown in Figure 4C, in HCT116, the percentage of cells with abnormal spindles increased from $12.1 \%$ in HCT116 ${ }^{\text {Empty }}$ clones to $19.7 \%$ in HCT116 ${ }^{\mathrm{BOP} 1}$ clones, $P=.0236$ (Figure 4C). Results from KM12SM cells were consistent, 21.1\% of KM12SMEmpty clones showed aberrant spindles, as compared to $40.3 \%$ of KM12SMBOP1 cells $P<.0001$ (Figure 4D). A greater percentage of cells with anaphase bridges was found in the HCT116 ${ }^{\mathrm{BOP} 1}$ clones when compared to controls, $49.0 \%$ vs 70.2\%, $P=.0036$ (Figure 4E). КM12SM ${ }^{\text {ВоР1 }}$ clones displayed a higher frequency of anaphase bridges compared to KM12SMEmpty $37.6 \%$ vs 63.0\%, $P<.0001$ (Figure 4E). Therefore, the CIN phenotype induced by BOP1 reproduced the one of its regulator, CCAT2.

\section{BOP1 plays an oncogenic role in CRC}

Functional assays were conducted to explore the role of BOP1 in CRC. Knocking-down BOP1 impaired the proliferation of HCT116 and KM12SM (Figure 5A). Consistently, the cell viability was higher in $\mathrm{HCT}_{116}^{\mathrm{BOP} 1}(P=.0024)$, and in $\mathrm{KM}^{2} \mathrm{SM}^{\mathrm{BOP} 1}(P=.0032)$ versus controls (Figure 5B). Cell colony formation was inhibited after knocking-down BOP1 in HCT116 $(P=.0156$ for siRNA 1 and $P=.0047$ for siRNA 2) (Figure 5C), whereas up-regulation of BOP1 promoted colony formation in HCT116 $(P=.0497)$ (Figure 5D) and KM12SM $(P=.0028)$ (Supplementary Figure 5A). Fewer cells migrated and invaded into the lower chamber in a transwell assay after BOP1 siRNAs, both in HCT116 $(P=.0175$ for siRNA 1 and $P<.0001$ for siRNA 2$)$ and KM12SM $(P=.0015$ for siRNA 1 and $P=.0038$ for siRNA 2) (Figure 5E). Stable over-expression of BOP1 enhanced cell migration and invasion in HCT116 $(P=.013)$ and KM12SM $(P=.0339)$ (Figure 5F). Scratch assays indicated that BOP1 positively regulates cell migration in HCT116: knocking-down BOP1 decreased migration $(P$ $=.0122$ for siRNA 1 and $P=.0031$ for siRNA 2) (Supplementary Figure 5B). Collectively, BOP1 protein plays an oncogenic role in CRC, confirming recent findings ${ }^{24}$ and the BOP1 overexpression phenotype mirrors the CCAT2 overexpression effects ${ }^{9}$.

\section{BOP1 modulates the function of AURKB}


We hypothesized that high levels of CCAT2 IncRNA would increase the level of ribosomal subunits and subsequently this could affect genomic integrity ${ }^{25}$. By performing polysome profiling, we concluded that the function of CCAT2 IncRNA via BOP1 is ribosome independent (Supplementary Figure 6A-6C).

In order to find down-stream targets of the CCAT2-BOP1 pathway, we used a genome-wide screen, CINdex analysis ${ }^{26}$, on the TCGA CRC cohort. We found that the aurora kinase family and PeBoW complex genes, associated with CIN and positively correlate with each other (Supplementary Figure 6D and Supplementary Table 9). This assay directed us to investigate aurora kinase family genes.

No difference in the levels of aurora kinase A (AURKA) protein, and of two other proteins reported to induce $\mathrm{CIN}, \mathrm{CDC} 20$, and BUB1B, were detected between scramble siRNA and CCAT2 knockdown in KM12SM (Supplementary Figure 6E) and BOP1 knockdown in HCT116 (Supplementary Figure 6F). We further checked for differences in the levels of aurora kinase B (AURKB) and phosphorylated aurora B (pAURKB) in HCT116 CCAT2 versus HCT116 Empty clones. We observed that the over-expression of CCAT2 induces higher pAURKB at Thr 232 (active form of AURKB), but no changes in AURKB mRNA and protein (Figure 6A left panel). In a second model, both total levels of AURKB mRNA and protein and pAURKB were higher in KM12SM compared to KM12C (Figure 6A right panel). AURKB is predominantly activated by autophosphorylation, and does not require the involvement of other kinase, while interaction with other molecules augments the phosphorylation ${ }^{27}$. Next, we observed that high BOP1 induces the phosphorylation of AURKB, both in HCT116 and KM12SM (Figure 6B) and the knock-down of BOP1 induced the downregulation of AURKB mRNA and protein and of pAURKB (Figure 6C). As MYC was reported to be a regulator of $A U R K B{ }^{28}$, we checked if MYC is a TF for AURKB. CHIP-Seq data (UCSC Genome Browser on Human Feb. 2009 (GRCh37/hg19) Assembly) showed that MYC binds to CCACGCC located in the 5' region of $A U R K B$ and acts as a TF for $A U R K B$ (Supplementary Figure 6G). This was confirmed in HCT116 MYC tet-on system: after induction of MYC, the levels of AURKB mRNA and protein and of pAURKB protein increased (Figure 6D).

Previously, it was shown that AURKB protein can bind mRNA molecules that stimulate AURKB activity during mitosis ${ }^{29}$. To identify whether CCAT2 RNA forms a complex with AURKB protein, we performed MS2-pull down assay: AURKB protein was retrieved in the CCAT2-MS2 but not in the MS2-empty vector transduced HCT116 cells (Figure 6E). By using RIP, we confirmed this 
complex: CCAT2 was detected 20 times higher in the AURKB precipitate compared to IgG control (P $<$.0001) (Supplementary Figure 6H). In order to determine which CCAT2 IncRNA segment associates with AURKB protein we did RNase I treatment of the lysate before RIP, so that unbound fragments were digested. Two of the segments were enriched: S8 and S6 (Supplementary Figure 6I).

To appreciate if the dysregulation of CCAT2-BOP1-AURKB pathway is widespread in cancers, we checked for this pathway in GC, which has high CIN rates $(50 \%)^{30}$. We performed a non-coding GEA comparing normal gastric samples with peritoneal carcinomatosis samples of GC patients (GSE133590). One of the top up-regulated IncRNA in peritoneal carcinomatosis was CCAT2 (P $=.0115$ ) (Supplementary Figure 7A). We analyzed the CCAT2 IncRNA expression in two pairs of GC patients derived xenografts (PDXs) and one GC patient derived organoid (PDO) and in each of them CCAT2 was up-regulated compared to parental cells (Supplementary Figure 7B), showing that successful engraftment, a marker of poor prognosis ${ }^{31}$, is associated with high CCAT2 levels. Next, we did CINdex analysis using the GC TCGA cohort and identified that aurora family and PeBoW complex genes positively correlated with CIN at chromosome level (Supplementary Figure 7B and Supplementary Table 10). Additionally, we used the primary GC cells, AGS, with euploid chromosomal number and KATO III with a tetraploid chromosome number. The RNA expression levels of CCAT2 and BOP1 and the protein levels of BOP1, the other components of the PeBoW complex and pAURKB were higher in KATO III compared to AGS (Supplementary Figure 7C). These data imply that the identified mechanism relates to CIN more generally.

\section{CCAT2 and BOP1 are overexpressed in MSS CRC}

To assess the clinical relevance of our findings, we examined multiple patient cohorts. Firstly, we used the TCGA CRC cohort (Cohort $\mathbf{A}$ ) as a screening cohort and identified significantly higher expression of BOP1, PES1, and WDR12 mRNAs in tumor versus normal tissue $(P<.0001)$ (Figure 7A). It is known that MSS/MSI-L CRC cancers (analyzed together as MSS) are CIN positive and MSI$\mathrm{H}$ (referred to as MSI) are CIN negative ${ }^{32}$. Therefore, we compared the mRNA expression of PeBoW complex components in MSS versus MSI CRC. Only the mRNA level of BOP1 was significantly higher in MSS versus MSI $(P<.0001)$ and PES1 and WDR12 showed no differences between the subgroups $(P=.8115$ and $P=.2333$ ) (Figure 7B). We were not able check for CCAT2 lncRNA in this cohort, as CCAT2 is not polyadenylated. 
In a second cohort of CRC tumors and paired adjacent normal tissues (Cohort B) we measured the RNA expression of CCAT2, BOP1, PES1, and WDR12: all were significantly overexpressed in tumor versus normal tissues (Supplementary Figure 8A). We also identified a significant positive correlation between CCAT2 IncRNA and BOP1 mRNA in tumor tissues $(\mathrm{r}=0.6296)$ (Supplementary Figure 8B). Regarding MSS versus MSI comparison, there were insufficient patients in the MSI group for this analysis. We identified that CCAT2 and BOP1 expression levels remained unchanged in MSI tumors versus paired normal tissues $(P=.7422$ and $P=.5649$, respectively), but significantly increased in MSS versus paired normal tissues $(P<.0001$ for both genes) (Supplementary Figure 8C).

In a third group, Cohort $\mathbf{C}$, we confirmed these data: CCAT2 IncRNA and the mRNA level of all three PeBoW components were highly expressed in tumor versus adjacent normal tissues $(P$ $<.0001$ ) (Figure 7C and Supplementary Figure 8D). We also checked for the correlation between BOP1 mRNA and CCAT2 IncRNA expression in normal and tumor tissues; the correlation between the two transcripts increased from $r=0.3050$ in normal tissues to an $r=0.5252$ in cancer (Supplementary Figure 8E and 8F). Because of an ample MSI sub-group, we compared the expression of CCAT2 lncRNA and PeBoW mRNAs between MSI and MSS. Only CCAT2 and BOP1, but not PES1 or WDR12, were up-regulated in MSS $(P=.001$ and $P=.03$, respectively) (Figure 7D and

\section{Supplementary Figure 8G).}

Additionally, we used Cohort D, with ample MSI subgroup, and we established that only CCAT2 and BOP1 were up-regulated in MSS versus MSI $(P<.0001$ and $P=.03$, respectively) (Figure 7E and Supplementary Figure 8H). The RNA expression levels of CCAT2 and BOP1 were positively correlated in tumor tissues $(\mathrm{r}=0.6263)$, implying co-regulation (Figure 7F). High levels of CCAT2 or BOP1 were associated with worse overall survival (OS) $(P<.0001$ and $P=.003$, respectively) (Figure 7G). Additionally, high levels of PES1 and WDR12 mRNAs were prognostic for shorter OS $(P=.0007$ and $P=.0004$, respectively) (Supplementary Figure 8I). Similarly, increased transcription levels of $C C A T 2$ and BOP1 predicted shorter recurrence free survival (RFS) $(P<.0001$ for both) (Figure 7H). PES1 and WDR12 proved to have a comparable prognostic value $(P<.0001$ and $P=.0002$, respectively) (Supplementary Figure 8J).

Cohort D had follow-up data for all patients. Univariate analysis revealed that high CCAT2 lncRNA and BOP1 mRNA levels were significantly associated with shorter OS and RFS (for CCAT2: HR: 6.1, 95\%CI: 2.79-13.31, $P<.0001$ and HR: 4.78, 95\%CI: 2.74-8.09, $P<.0001$, respectively; for 
BOP1: HR: 3.01, 95\%CI: 1.38-6.55, $P=.006$ and HR: 2.77, 95\%CI: $1.65-4.60, P=.0002$, respectively) (Supplementary Table 11). Multivariate analysis revealed that high CCAT2 and BOP1 were independent factors for predicting poor OS and RFS (for CCAT2: HR: 5.51, 95\%CI: 2.4812.27, $P<.0001$ and HR: 4.85, 95\% CI: 2.76-8.28, $P<.0001$, respectively; for BOP1: HR: 3.12, 95\%CI: 1.42-6.84, $P=.005$ and HR: 2.67, 95\%CI: 1.59-4.45, $P=.0003$, respectively) (Supplementary Table 12).

Finally, to assess if our findings are present at the protein level, we used Cohort E. We previously showed that the expression of CCAT2 is higher in tumor tissues of each of these patients compared to their normal tissue ${ }^{10}$. We assessed the protein expression of BOP1, AURKB, and pAURKB and identified high protein levels of BOP1 and pAURKB in tumor tissue compared to normal mucosae for $60 \%(6 / 10)$ of the pairs, all six being MSS CRC (Figure 7I). We also identified that CCAT2 IncRNA and BOP1 protein are upregulated in MSS PDX compared to MSI PDX (Supplementary Figure 8K). Collectively, these results prove that CCAT2 and the PeBoW complex are oncogenes and only CCAT2 and BOP1 are specifically overexpressed in MSS CRC.

\section{Discussion}

CIN correlates with patient survival in CRC 33 and multiple other cancers ${ }^{34}$. Therefore, understanding the mechanisms underpinning CIN is essential to discover new therapies. Dozens of proteins are involved in chromosome segregation ${ }^{35}$. Many of them have recently been evaluated as therapeutic targets, but none of the proposed treatments have reached approval. We propose that targeting ncRNAs involved in chromosomal segregation errors might provide an alternative approach to inhibiting CIN. We present compelling new data supporting the involvement of the IncRNA CCAT2 in the development of CIN.

First, we showed that high CCAT2 IncRNA is sufficient to induce early premalignant modifications. CCAT2 mice, after AOM/DSS treatment, have significantly more polyps and the degree of colon glands' hyperplasia and dysplasia is higher compared to WT mice. Additionally, a high CCAT2 background induces CIN in organoids established from healthy mouse colon, before tumor formation. We demonstrated that CCAT2 IncRNA induces CIN via BOP1 in two ways: through an "indirect transcriptional mechanism", probably via c-MYC, a TF promoting BOP1 gene expression, and by a "direct post-transcriptional mechanism", by binding the mature BOP1 protein, prolonging its half-life. The genes within this pathway (CCAT2, MYC, and BOP1) are located on the 
well-studied, oncogenic chromosomal amplicon 8q24. In support of our experimental data, we showed that BOP1 mRNA and CCAT2 IncRNA expression correlate positively, in CRC patient cohorts. These results led us to the conclusion that CCAT2 lncRNA is an important factor that induces the up-regulation of BOP1 in cancer and promotes CIN.

Second, we discovered that CCAT2 lncRNA and BOP1 are abnormal in three cancer models, CRC, GC, and in the in vivo CCAT2 transgenic mice treated with AOM/DSS. These results suggest that the new mechanism identified could represents a general model of CIN initiation.

Third, by using one independent genome-wide screening method - CINdex in TCGA CRC cohort, we hypothesized that CCAT2 lncRNA via BOP1 induces CIN through aurora kinase family proteins. Indeed, the expression of CCAT2 lncRNA or BOP1 correlated with the phosphorylation of AURKB suggesting that CCAT2 lncRNA acts as an adaptor, promoting interaction of BOP1 and AURKB, bringing them in physical proximity. Together, our findings concerning the CCAT2 - BOP1 - AURKB pathway expression and function describe a new mechanism of CIN (Figure 7J).

NcRNAs and ribosomal biogenesis proteins have rarely been studied in the context of CIN. Several studies have emerged reporting BOP1 as an oncogene that can induce CIN. Killian et al. showed that the depletion of BOP1 in CRC cells increased the number of aberrant mitotic cells ${ }^{19}$. The same group analyzed the expression of BOP1 in CRC patients and observed that high BOP1 was tumor specific. Other, studies revealed that BOP1 overexpression increased the number of multipolar spindles ${ }^{18}$. In rectal cancer patients, $B O P 1$ was shown to be overexpressed in samples with gain of the $8 \mathrm{q}$ chromosome arm and steadily increased from adenoma to carcinoma, implying a tumorigenic role ${ }^{36}$. None of the studies explored the upstream pathways that regulate BOP1, or the precise mechanism by which BOP1 induces CIN. On the other hand, AURKB, is one of the key regulators of mitosis ${ }^{37}$, increased activation of AURKB is present in multiple cancer types and correlates with $\mathrm{CIN}$ 38. The variety of interactions that facilitate the post-transcriptional modifications that activate AURKB are not fully deciphered. Hence, it is crucial to understand new mechanisms that regulate AURKB function and CCAT2 via BOP1 appears to be a novel pathway activating AURKB.

In conclusion, this study presents a new mechanism in which the lncRNA, CCAT2, induces CIN, an early tumorigenic event. This pathway reveals new potential therapeutic targets for CIN. 


\section{Figure Legends}

Figure 1. CCAT2 IncRNA induces CIN and activates pathways associated with ribosomal proteins. (A) Cytogenetic analysis showing chromosomal aberrations in HCT116 ${ }^{\text {CCAT2 }}$ cells (left) and KM12SM cells (right). Red arrows indicate breaks, blue arrows indicate fusions and green arrows indicate fragments. (B) The frequency of aberrant metaphases in HCT116Empty versus HCT116CCAT2 and KM12C versus KM12SM. At least 35 metaphases were analyzed for each clone. (C) Immunofluorescence images and (D) frequency of abnormal spindle (upper lane) and anaphase bridge (lower lane) in HCT116CCAT2 cells. At least 200 interphase cells were analyzed for each clone. (E) Cytogenetic analysis showing chromosomal aberrations in organoids from WT (left) and CCAT2 mice (right). Blue arrow indicates fusions. (F) The frequency of aberrant metaphases in organoids from WT versus CCAT2 mice. At least 35 metaphases were analyzed for each organoid. (G) Schematic illustration of the AOM/DSS colon cancer model. (H) Images of colon mucosa from WT and CCAT2 mice after treatment with AOM/DSS. Red delineation indicates polyps' area. (I) Total number of polyps, total surface area of colon polyps, and average polyp diameter size in WT and CCAT2 transgenic mice at the end of the AOM/DSS treatment ( $\mathrm{n}=7$ per group). (J) H\&E images of the colon from WT and CCAT2 transgenic mice after treatment with AOM/DSS. Black arrows indicate polyps. (K) Largest size polyp according to H\&E analysis in WT and CCAT2 transgenic mice. (L) The percentage of WT and CCAT2 transgenic mice with grade 1-2 versus grade 3-4 hyperplasia, and (M) with normal glands versus dysplastic glands. (N) IPA analysis showing significantly enriched pathways in CCAT2 transgenic mouse model (left Y axis represents negative $\log P$ values; right $Y$ axis represents the ratio of molecules in the dataset mapping to the number of molecules in the canonical pathways) (left panel). Venn diagram showing overlapping genes related to the ribosomal proteins from the canonical pathways (right panel). Mean \pm SD. $(* * P<.01)$.

Figure 2. CCAT2 IncRNA interact with BOP1. (A) Schematic illustration of MS2-pull down assay (left panel) and immuno-blotting results of BOP1, PES1, and WDR12 (right panel). (B) RIP assay was performed to check the enrichment of CCAT2 IncRNA in COLO320. (C) In vitro RNA pull-down using GST-tagged CCAT2 lncRNA and recombinant BOP1 protein. (D) Determination of the interaction between $\triangle \mathrm{BOP} 1$ domains and CCAT2 lncRNA by in vitro RNA pull down. (E) In vitro RNA pull-down using multiple CCAT2 lncRNA segments (S1 to S10). (F) SHAPE assay showing the 
structure of the CCAT2 IncRNA region from nucleotide 207 to 492 . Blue arrows indicate the start and the end of segment 3 . Mean \pm SD. $\left({ }^{*} P<.05\right),\left({ }^{* *} P<.01\right)$.

Figure 3. CCAT2 IncRNA up-regulates BOP1 in vitro and in vivo. (A) Expression of PeBoW complex components in HCT116 Empty and HCT116 ${ }^{\text {CCAT2 }}$ (left panel), and in KM12C and KM12SM (right panel). (B) Expression of PeBoW complex components in HCT116 WT (left panel) and KM12SM (right panel) after CCAT2 knock-down. (C) The half-life of BOP1 protein in KM12SM cells with transient CCAT2 overexpression or empty vector (left panel). Data from three experiments were quantified and are depicted as a graphic (right panel). (D) The nuclear and cytoplasmic localization of PeBoW complex in HCT116Empty and HCT116CCAT2 (E) Expression of CCAT2 lncRNA and PeBoW complex components in the colon of CCAT2 mice. (F) The expression of BOP1 in normal colon tissues and colon polyps of CCAT2 mice after AOM/DSS treatment $(n=7)$. Mean \pm SD. (ns, not significant), $\left({ }^{*} P<.05\right),\left({ }^{* *} P<.01\right) ;\left({ }^{* *} P<.001\right),\left({ }^{* * * *} P<.0001\right)$.

Figure 4. Overexpression of BOP1 promotes CIN. (A) Cytogenetic analysis showing chromosomal aberrations in cells with overexpression of BOP1. In panels (i), (ii) and (iii) are representative images of HCT116 ${ }^{\mathrm{BOP} 1}$, with fusion (blue arrow), break (red arrow), and fragments (green arrows). In panel (iv) is an image of KM12SM ${ }^{\text {BoP1 }}$ with polyploidy and acentric chromosomes (black arrows) and fusion (blue arrow), and in panel (v) is an image of HT29Bop1 with c-anaphase morphology. (B) The frequency of cells exhibiting chromosome abnormalities in HCT116, KM12SM, and HT29 Empty versus BOP1 overexpressed clones. At least 35 metaphases were analyzed for each clone. (C, D) Images and frequencies of abnormal spindles in HCT116 (C) and KM12SM (D) with BOP1 overexpression. At least 200 interphase nuclei were analyzed for each clone. (E, F) Images and frequency of anaphase bridges in HCT116 (E) and KM12SM (F) with BOP1 overexpression. At least 200 cells were analyzed for each clone. Mean \pm SD. $\left({ }^{*} P<.05\right),\left({ }^{* *} P\right.$ $<.01),\left({ }^{* * * *} P<.0001\right)$.

Figure 5. BOP1 plays an oncogenic role in CRC. (A) Proliferation rate of HCT116 (left) and KM12SM (right) after siRNA knock-down of BOP1. (B) Proliferation rate of HCT116 ${ }^{\text {Empty }}$ and HCT116 ${ }^{\mathrm{BOP} 1}$ (left) and KM12SMEmpty and KM12SMBOP1 (right). (C, D) Representative images of 
colony formation assay in HCT116 with BOP1 knock-down (C) and HCT116 with stable overexpression of BOP1 (D). Quantitative analysis of colony numbers (right side of panels C and D). (E) Invasion potential of HCT116 and KM12SM cells after transfection with BOP1 siRNA. Representative images of invasion assay for HCT116 (upper panel) and KM12SM (lower panel). Quantitative analysis of invading cell (right panel). (F) Invasion potential in cells with stable overexpression of BOP1. Representative images of invasion assay for HCT116 (upper panel) and KM12SM (lower panel). Quantitative analysis of invading cell (right panel). Mean \pm SD. $\left({ }^{*} P<.05\right)$, $\left({ }^{* *} P<.01\right) ;\left({ }^{* * *} P<.001\right),\left({ }^{* * * *} P<.0001\right)$.

Figure 6. BOP1 modulates the function of AURKB. (A) Expression of AURKB and pAURKB in HCT116 Empty and HCT116CCAT2 (left panel) and KM12C and KM12SM (right panel). (B) Expression of BOP1, AURKB, and pAURKB analyzed in HCT116 Empty and HCT116 ${ }^{\text {BOP1 }}$ (left panel) and KM12SMEmpty and KM12SMBOP1 (right panel). (C) Expression of BOP1, AURKB, and pAURKB analyzed in HCT116 (left panel) and KM12SM (right panel) after BOP1 knock-down with siRNA. (D) Expression of BOP1 and AURKB at 0,6,12, 18, and 24 hours in HCT116 cells with inducible cMYC expression system. (E) MS2-pull down assay to identify if AURKB interacts with MS2-labeled CCAT2 in HCT116. Mean \pm SD. (ns, not significant), $\left({ }^{* *} P<.01\right) ;\left({ }^{* * *} P<.001\right),\left({ }^{* * * *} P<.0001\right)$.

Figure 7. CCAT2 and BOP1 are overexpressed in MSS CRC. (A) The expression levels of PeBoW complex in Cohort A. (B) The expression levels of the PeBoW complex in MSI and MSS primary CRC in Cohort A. (C) The expression of CCAT2 lncRNA and BOP1 mRNA in tumor and adjacent normal tissues from Cohort C. (D) The expression of CCAT2 IncRNA and BOP1 mRNA in MSI and MSS CRC from Cohort C. (E) The expression of CCAT2 IncRNA and BOP1 mRNA in MSI and MSS CRC from Cohort D. (F) Correlation between the RNA expression of CCAT2 and BOP1 in patients from Cohort D. (G) Kaplan-Meier OS curves of CRC patients from Cohort D, CCAT2 lncRNA (left panel) and BOP1 mRNA (right panel). (H) Kaplan-Meier RFS curves of CRC patients from Cohort D, of CCAT2 lncRNA (left panel) and BOP1 mRNA (right panel). Time is expressed in days. (I) Western blot analysis of BOP1, AURKB, and pAURKB protein expression in paired CRC samples (Cohort E). $\mathrm{N}=$ normal tissue, $\mathrm{T}=$ tumor tissue, $\mathrm{N} / \mathrm{A}=$ not available microsatellite status. The samples in which both BOP1 and pAURKB proteins are up-regulated in tumor versus normal tissues are marked with red stars. (J) A model of CCAT2 involvement in CIN (red arrows - new interactions; black 
arrows - available data). Data are represented as violin plots. (ns) not significant, $\left({ }^{*} P<.05\right),\left({ }^{* *} P\right.$ $<.01) ;(* * *<.001),\left({ }^{* * *} P<.0001\right)$. 


\section{References}

1. Gordon DJ, Resio B, Pellman D. Causes and consequences of aneuploidy in cancer. Nat Rev Genet 2012;13:189-203.

2. Boveri M. Über Mitosen bei einseitiger Chromosomenbindung. Jenaische Zeitschrift für Naturwissenschaft 1903;37:401-443.

3. Negrini S, Gorgoulis VG, Halazonetis TD. Genomic instability--an evolving hallmark of cancer. Nat Rev Mol Cell Biol 2010;11:220-8.

4. Gronroos E, Lopez-Garcia C. Tolerance of Chromosomal Instability in Cancer: Mechanisms and Therapeutic Opportunities. Cancer Res 2018;78:6529-6535.

5. Galimberti F, Thompson SL, Ravi S, et al. Anaphase catastrophe is a target for cancer therapy. Clin Cancer Res 2011;17:1218-22.

6. Hoffelder DR, Luo L, Burke NA, et al. Resolution of anaphase bridges in cancer cells. Chromosoma 2004;112:389-97.

7. Bakhoum SF, Cantley LC. The Multifaceted Role of Chromosomal Instability in Cancer and Its Microenvironment. Cell 2018;174:1347-1360.

8. Dragomir MP, Kopetz S, Ajani JA, et al. Non-coding RNAs in GI cancers: from cancer hallmarks to clinical utility. Gut 2020;69:748-763.

9. Ling H, Spizzo R, Atlasi Y, et al. CCAT2, a novel noncoding RNA mapping to 8q24, underlies metastatic progression and chromosomal instability in colon cancer. Genome Res 2013;23:1446-61.

10. Redis RS, Vela LE, Lu W, et al. Allele-Specific Reprogramming of Cancer Metabolism by the Long Noncoding RNA CCAT2. Mol Cell 2016;61:520-534.

11. Shah MY, Ferracin M, Pileczki V, et al. Cancer-associated rs6983267 SNP and its accompanying long noncoding RNA CCAT2 induce myeloid malignancies via unique SNP-specific RNA mutations. Genome Res 2018;28:432-447.

12. Pellagatti A, Boultwood J. The molecular pathogenesis of the myelodysplastic syndromes. Eur J Haematol 2015;95:3-15.

13. Maslov AY, Vijg J. Genome instability, cancer and aging. Biochim Biophys Acta 2009;1790:963-9.

14. Bayani J, Selvarajah S, Maire G, et al. Genomic mechanisms and measurement of structural and numerical instability in cancer cells. Seminars in Cancer Biology 2007;17:5-18.

15. Gerling M, Glauben R, Habermann JK, et al. Characterization of chromosomal instability in murine colitisassociated colorectal cancer. PLoS One 2011;6:e22114.

16. Roux PP, Topisirovic I. Regulation of mRNA translation by signaling pathways. Cold Spring Harb Perspect Biol 2012;4.

17. Kim TH, Leslie P, Zhang Y. Ribosomal proteins as unrevealed caretakers for cellular stress and genomic instability. Oncotarget 2014;5:860-71.

18. Killian A, Sarafan-Vasseur N, Sesboue R, et al. Contribution of the BOP1 gene, located on 8q24, to colorectal tumorigenesis. Genes Chromosomes Cancer 2006;45:874-81.

19. Killian A, Le Meur N, Sesboue R, et al. Inactivation of the RRB1-Pescadillo pathway involved in ribosome biogenesis induces chromosomal instability. Oncogene 2004;23:8597-602.

20. Beroukhim R, Mermel $\mathbf{C H}$, Porter $\mathrm{D}$, et al. The landscape of somatic copy-number alteration across human cancers. Nature 2010;463:899-905.

21. Rohrmoser M, Holzel M, Grimm T, et al. Interdependence of Pes1, Bop1, and WDR12 controls nucleolar localization and assembly of the PeBoW complex required for maturation of the 605 ribosomal subunit. Mol Cell Biol 2007;27:3682-94.

22. Neer EJ, Schmidt CJ, Nambudripad R, et al. The ancient regulatory-protein family of WD-repeat proteins. Nature 1994;371:297-300.

23. Kent WJ, Sugnet CW, Furey TS, et al. The human genome browser at UCSC. Genome Res 2002;12:9961006. 
24. Qi J, Yu Y, Akilli Ozturk O, et al. New Wnt/beta-catenin target genes promote experimental metastasis and migration of colorectal cancer cells through different signals. Gut 2016;65:1690-701.

25. Grummt I. The nucleolus-guardian of cellular homeostasis and genome integrity. Chromosoma 2013;122:487-97.

26. Song L, Bhuvaneshwar K, Wang Y, et al. CINdex: A Bioconductor Package for Analysis of Chromosome Instability in DNA Copy Number Data. Cancer Inform 2017;16:1176935117746637.

27. Yasui $Y$, Urano $T$, Kawajiri $A$, et al. Autophosphorylation of a newly identified site of Aurora-B is indispensable for cytokinesis. J Biol Chem 2004;279:12997-3003.

28. den Hollander J, Rimpi S, Doherty JR, et al. Aurora kinases A and B are up-regulated by Myc and are essential for maintenance of the malignant state. Blood 2010;116:1498-505.

29. Jambhekar A, Emerman AB, Schweidenback CT, et al. RNA stimulates Aurora B kinase activity during mitosis. PLoS One 2014;9:e100748.

30. Cancer Genome Atlas Research N. Comprehensive molecular characterization of gastric adenocarcinoma. Nature 2014;513:202-9.

31. DeRose YS, Wang G, Lin YC, et al. Tumor grafts derived from women with breast cancer authentically reflect tumor pathology, growth, metastasis and disease outcomes. Nat Med 2011;17:1514-20.

32. Simons CC, Hughes LA, Smits KM, et al. A novel classification of colorectal tumors based on microsatellite instability, the CpG island methylator phenotype and chromosomal instability: implications for prognosis. Ann Oncol 2013;24:2048-56.

33. Watanabe T, Kobunai T, Yamamoto $\mathrm{Y}$, et al. Chromosomal instability (CIN) phenotype, CIN high or CIN low, predicts survival for colorectal cancer. J Clin Oncol 2012;30:2256-64.

34. Jamal-Hanjani $\mathbf{M}$, Wilson GA, McGranahan N, et al. Tracking the Evolution of Non-Small-Cell Lung Cancer. N Engl J Med 2017;376:2109-2121.

35. Carter SL, Eklund AC, Kohane IS, et al. A signature of chromosomal instability inferred from gene expression profiles predicts clinical outcome in multiple human cancers. Nature Genetics 2006;38:10431048.

36. Lips EH, van Eijk R, de Graaf EJ, et al. Integrating chromosomal aberrations and gene expression profiles to dissect rectal tumorigenesis. BMC Cancer 2008;8:314.

37. Carmena $\mathbf{M}$, Wheelock $\mathbf{M}$, Funabiki $\mathrm{H}$, et al. The chromosomal passenger complex (CPC): from easy rider to the godfather of mitosis. Nat Rev Mol Cell Biol 2012;13:789-803.

38. Munoz-Barrera M, Monje-Casas F. Increased Aurora B activity causes continuous disruption of kinetochore-microtubule attachments and spindle instability. Proc Natl Acad Sci U S A 2014;111:E39964005. 
Figure 1

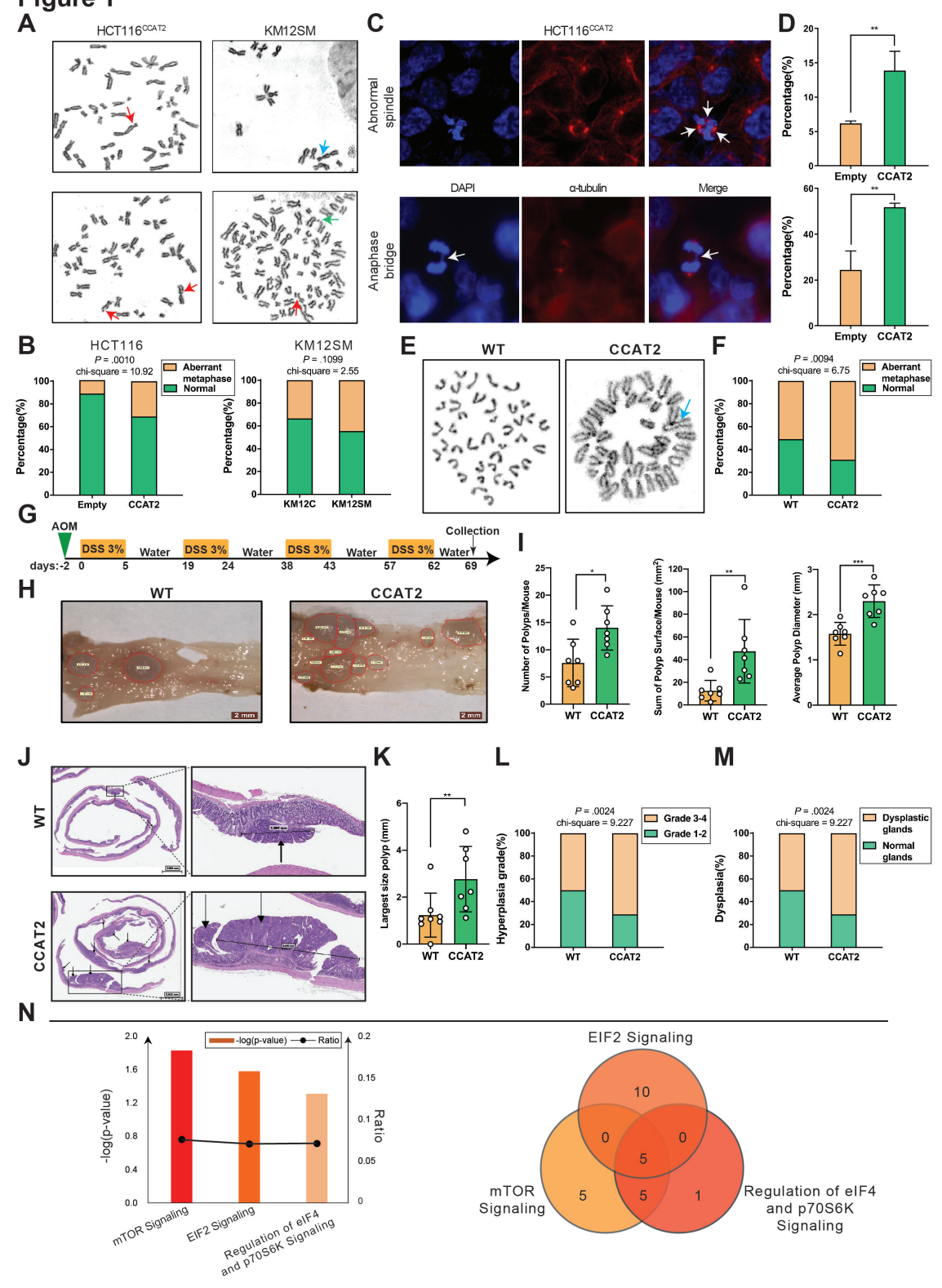




\section{Figure 2}
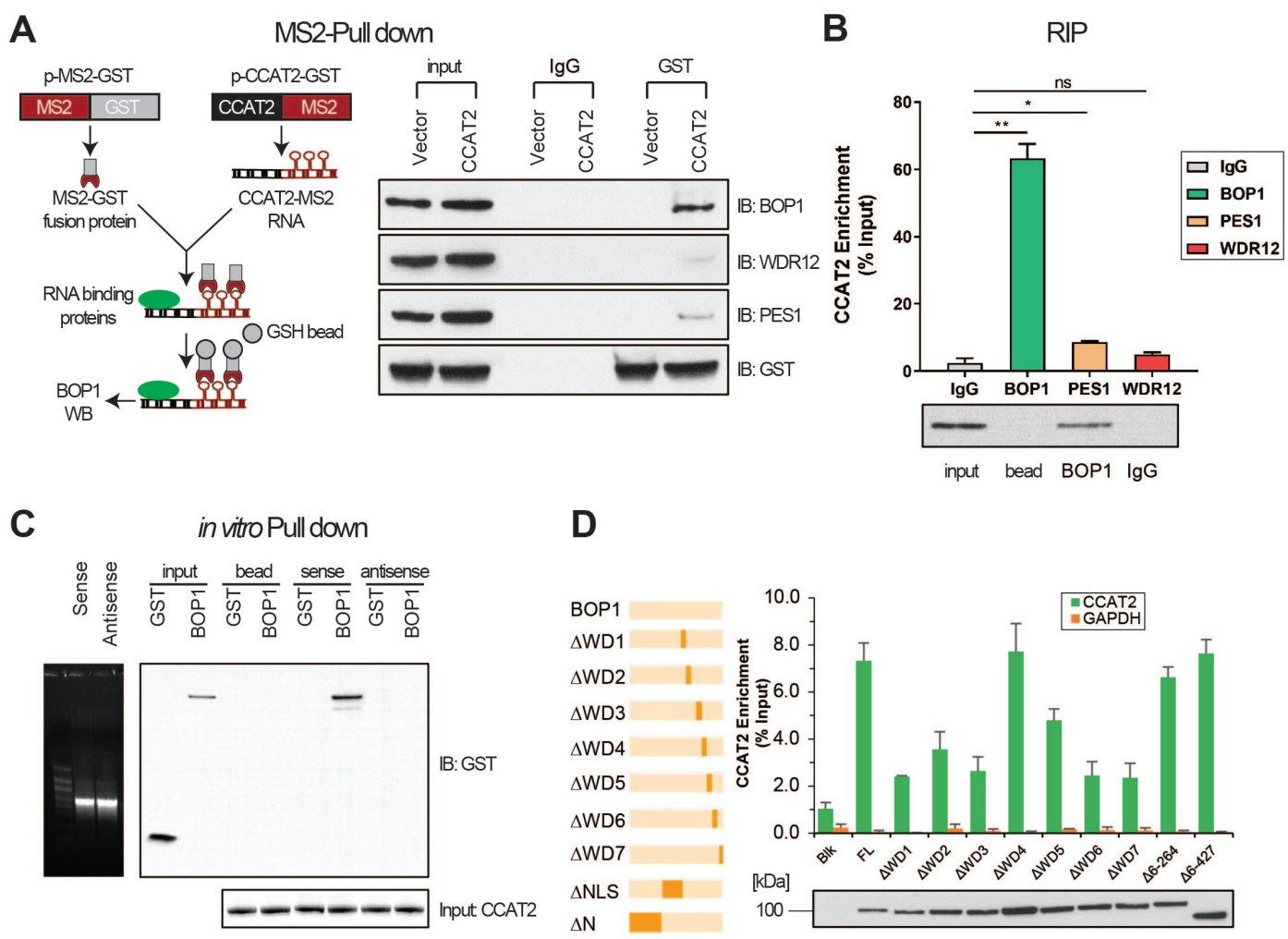

D

E

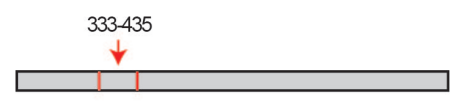

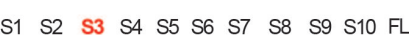

-

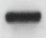

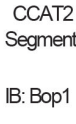

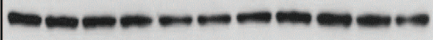

Input $\gamma$-Tubulin

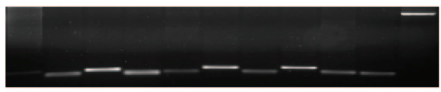

in vitro

transcription

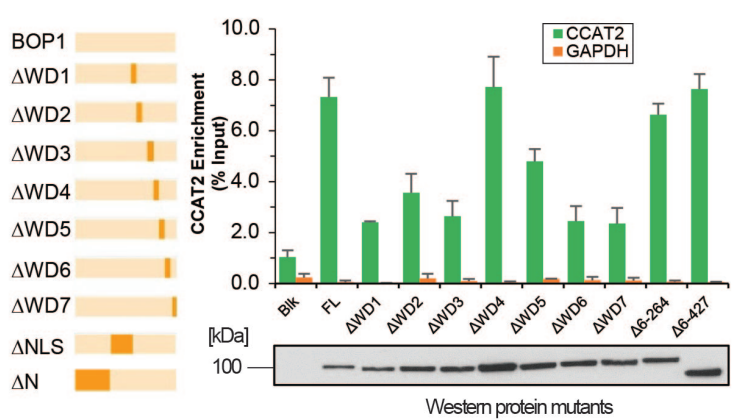

F

SHAPE

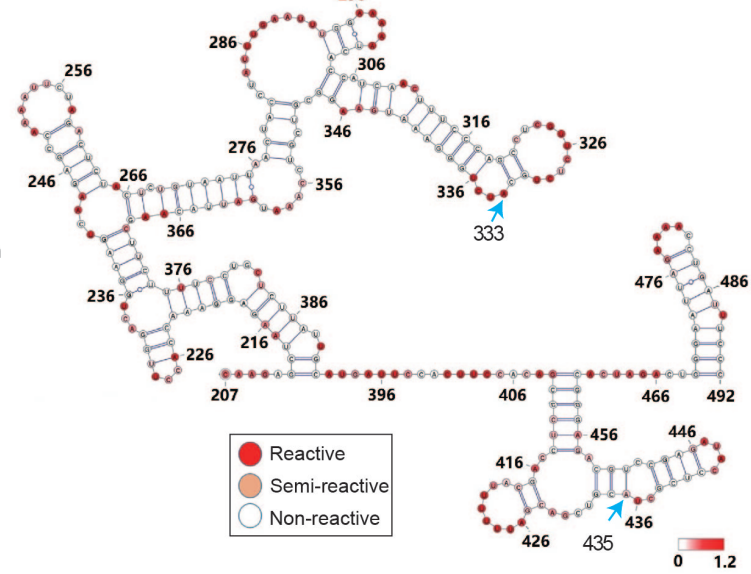


Figure 3
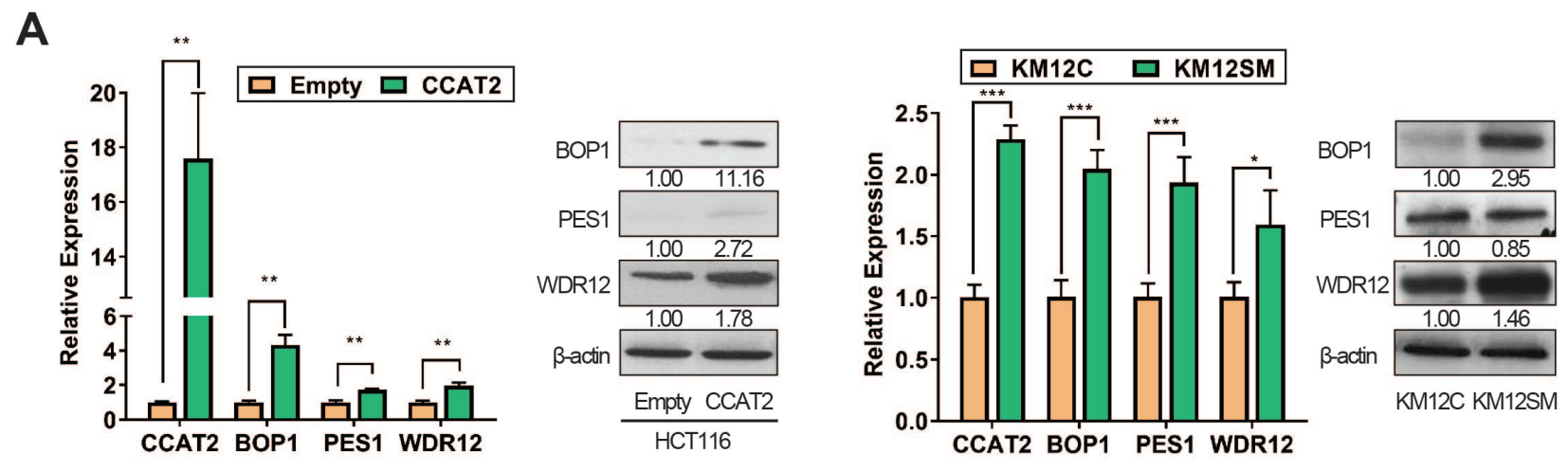

B

HCT116WT

KM12SM
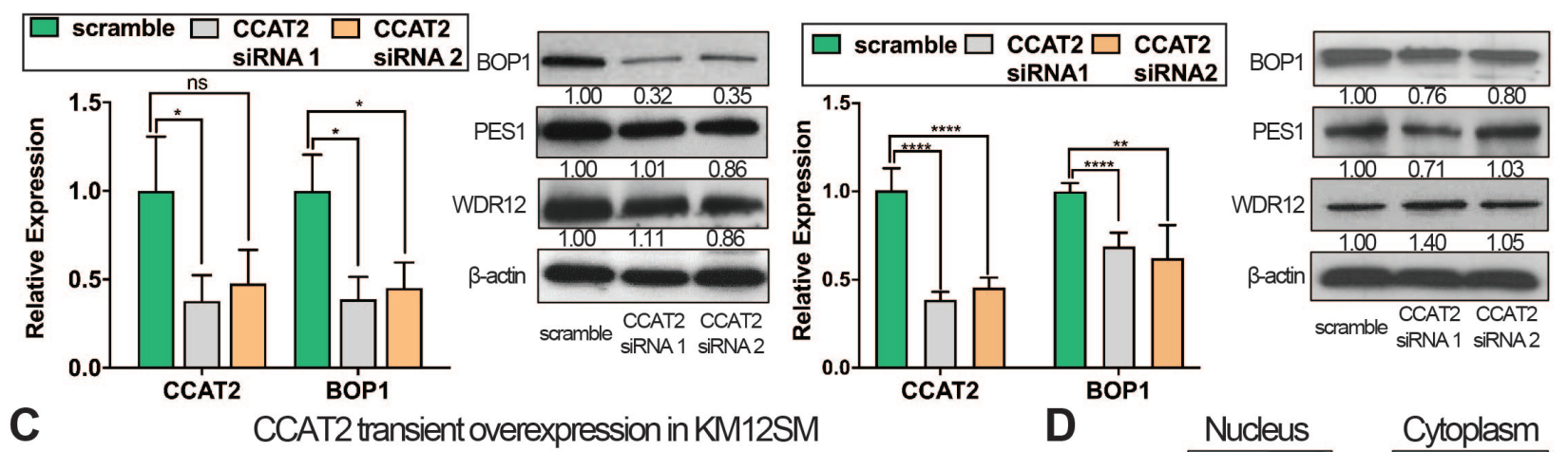

C

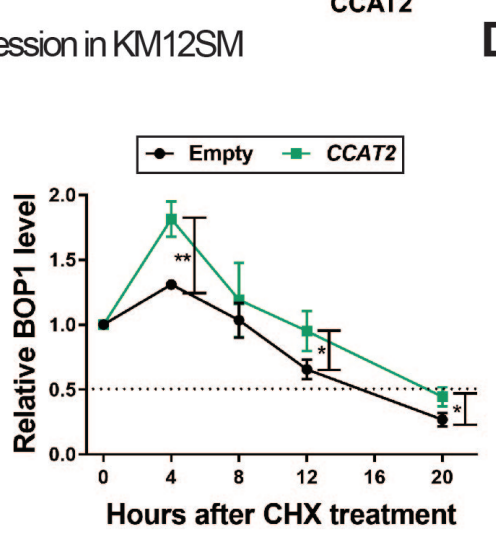

D

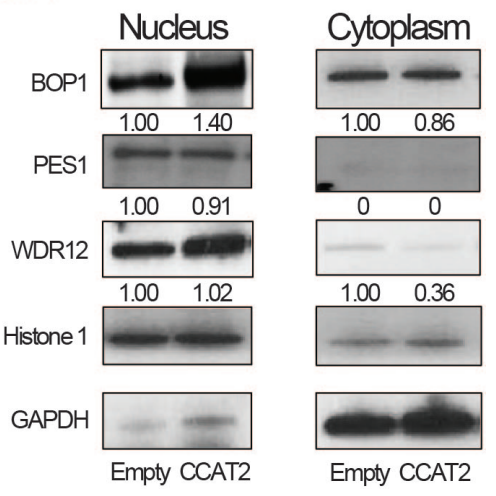

E

Mouse colon

3

F

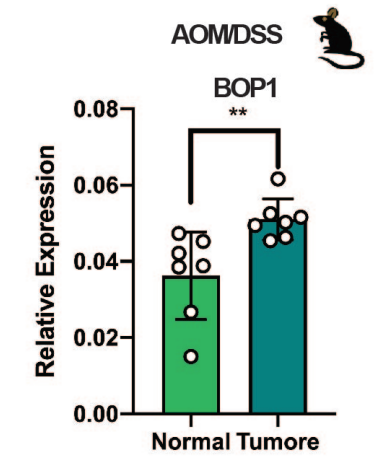


Figure 4

A

cytogenetics
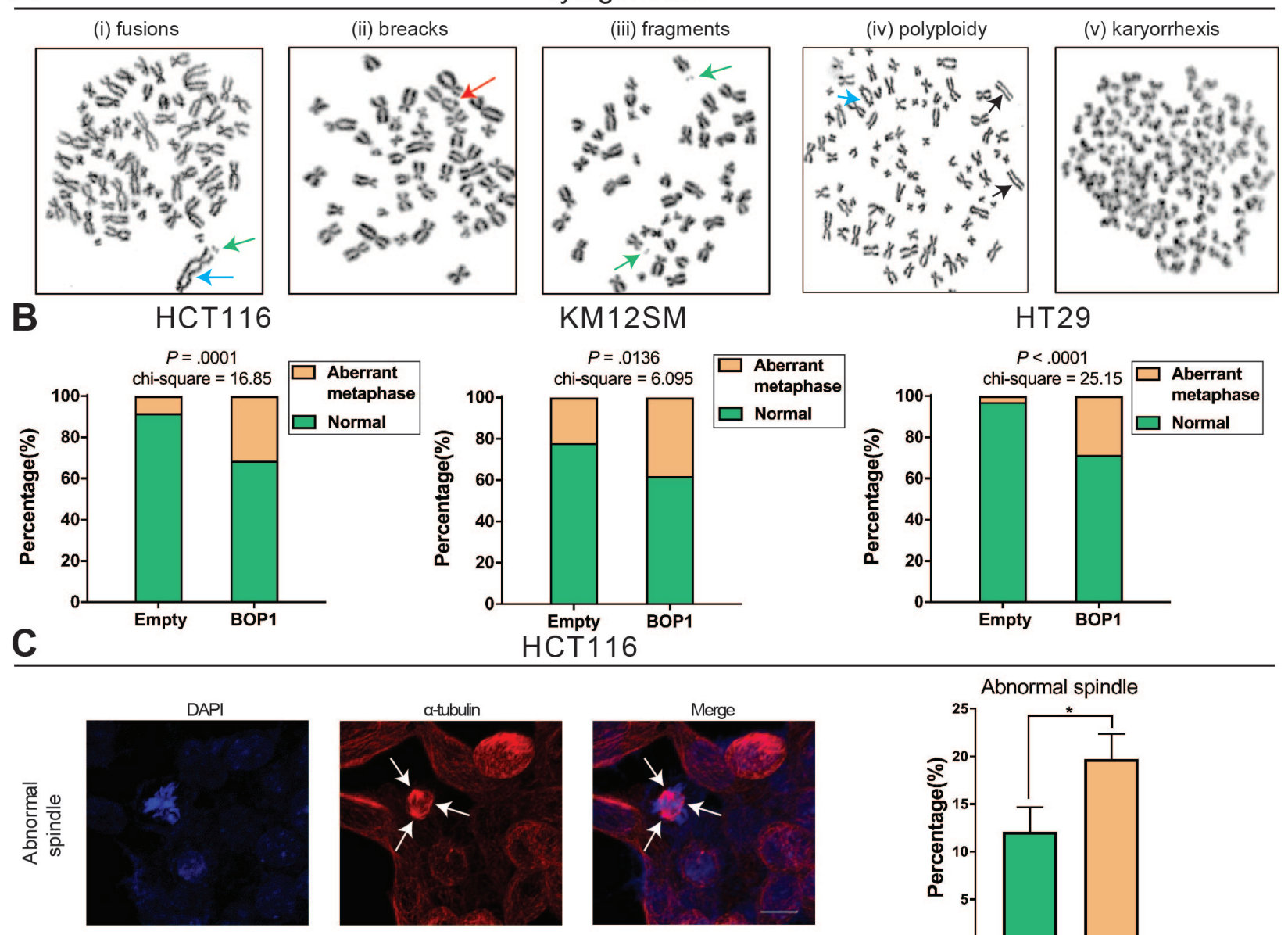

D

KM12SM
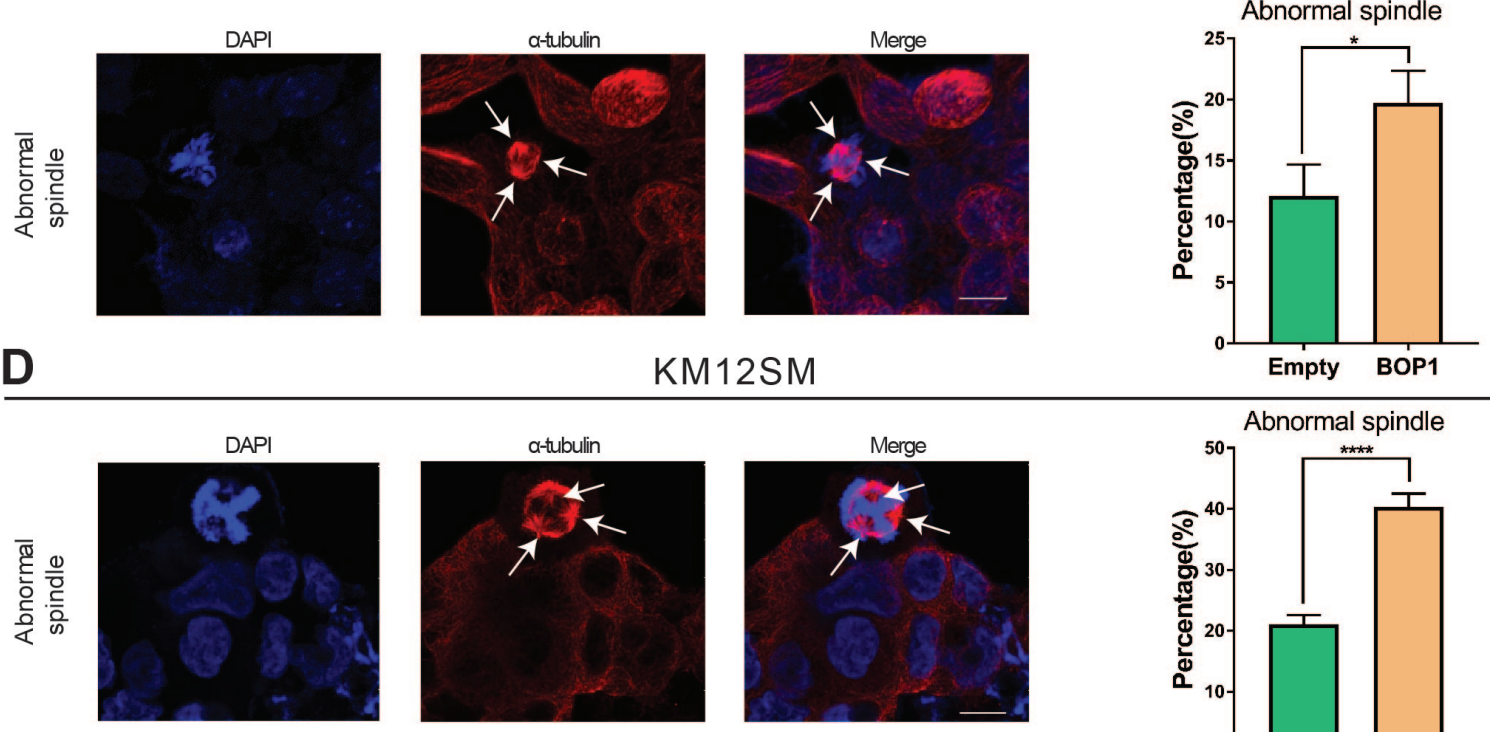

E

HCT116

F
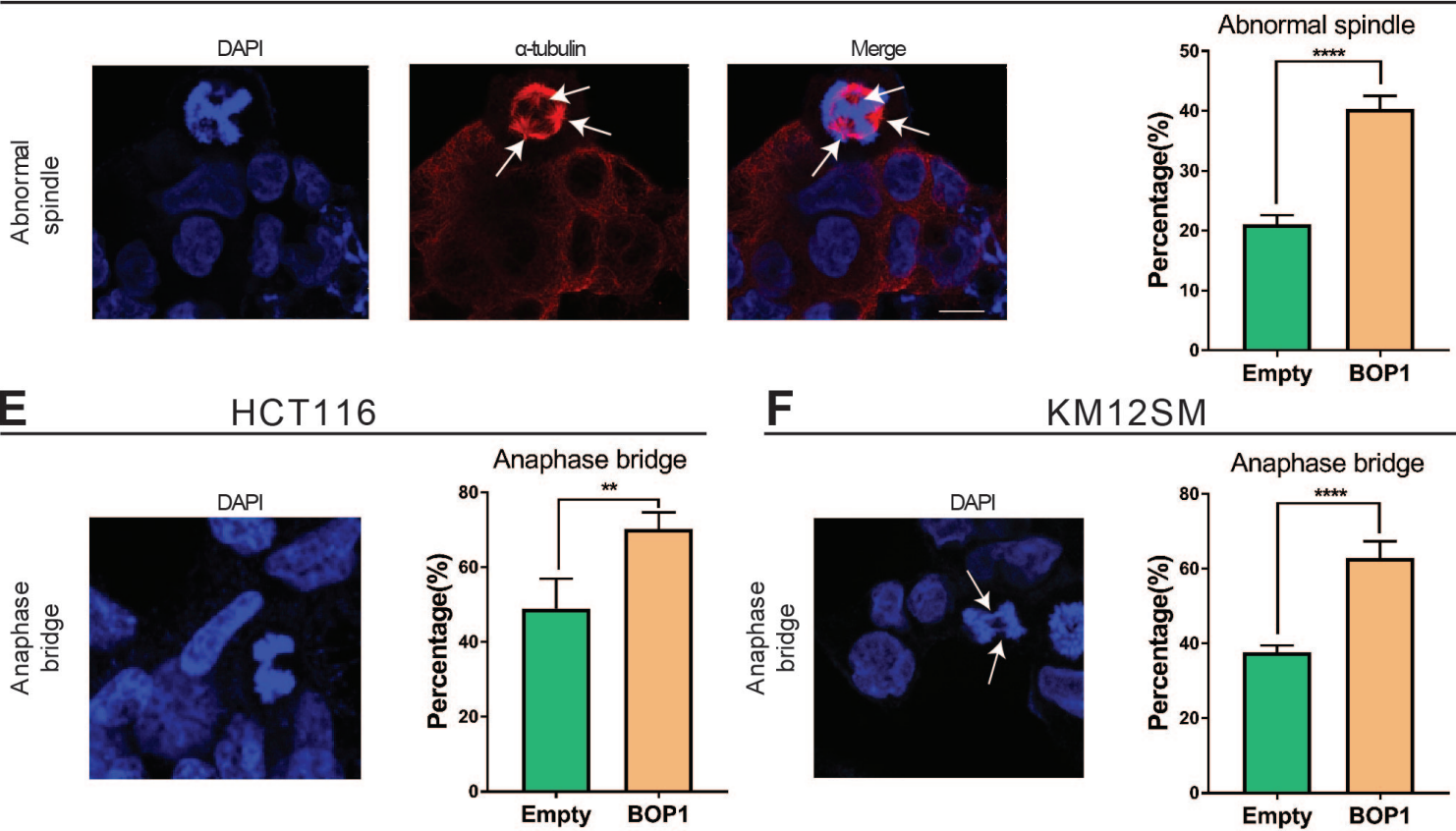


\section{Figure 5}
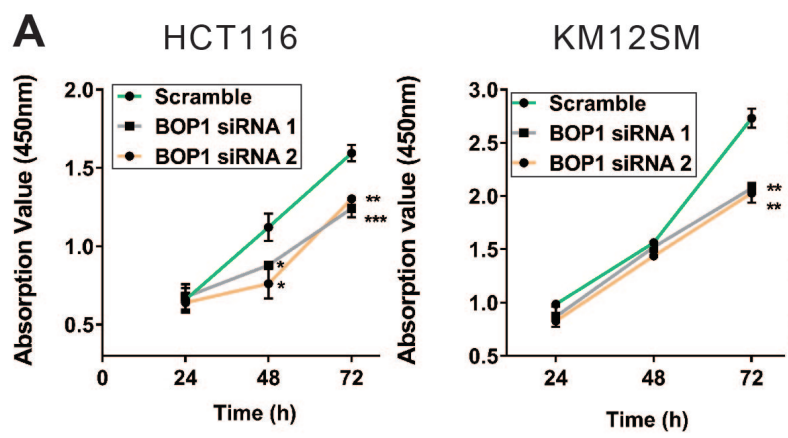

B

HCT116

KM12SM

C

HCT116
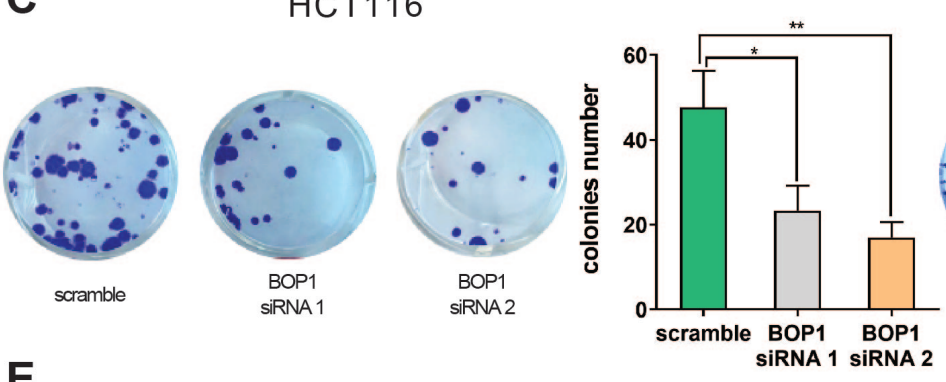

D

HCT116

E
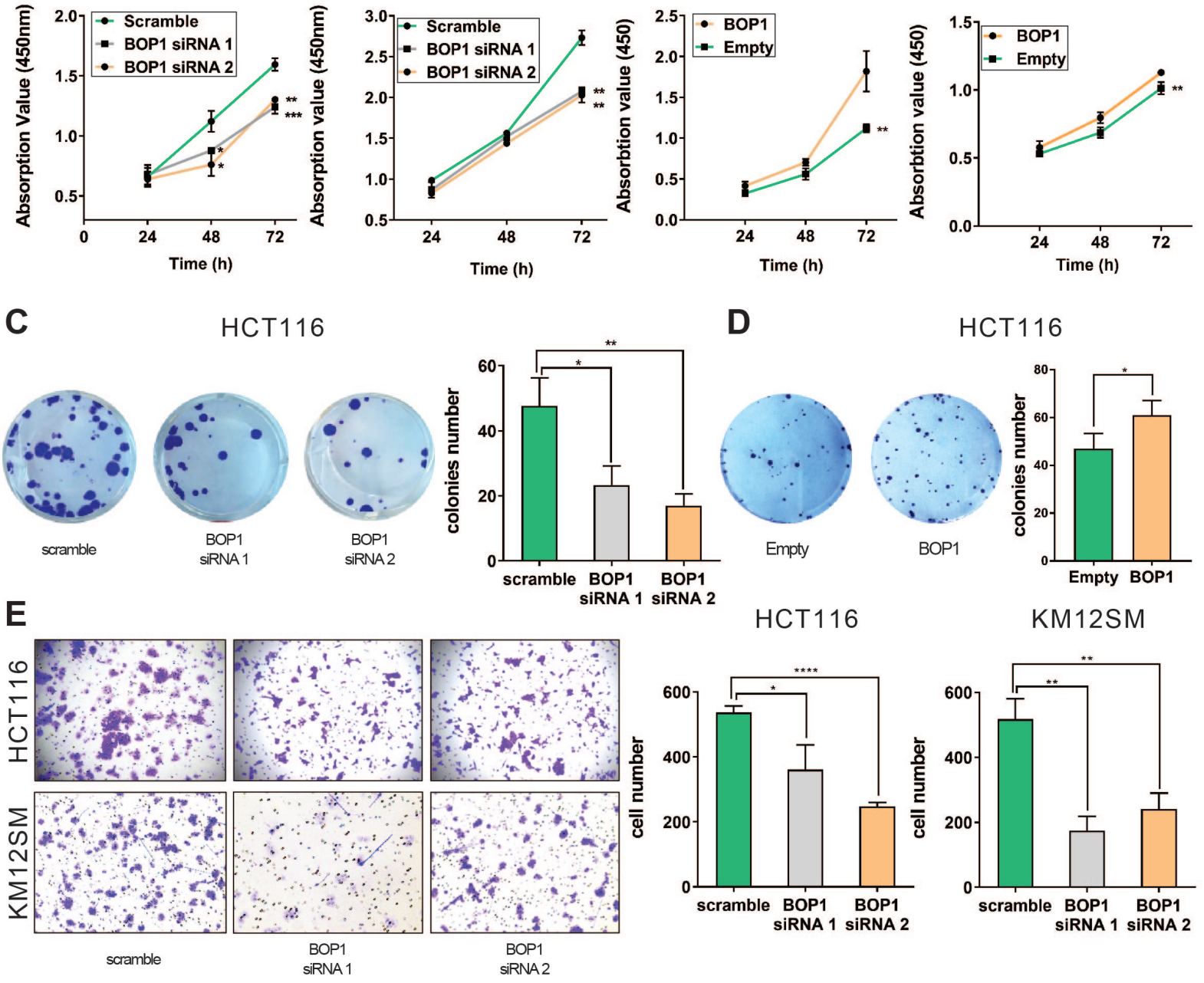

F

HCT116

KM12SM
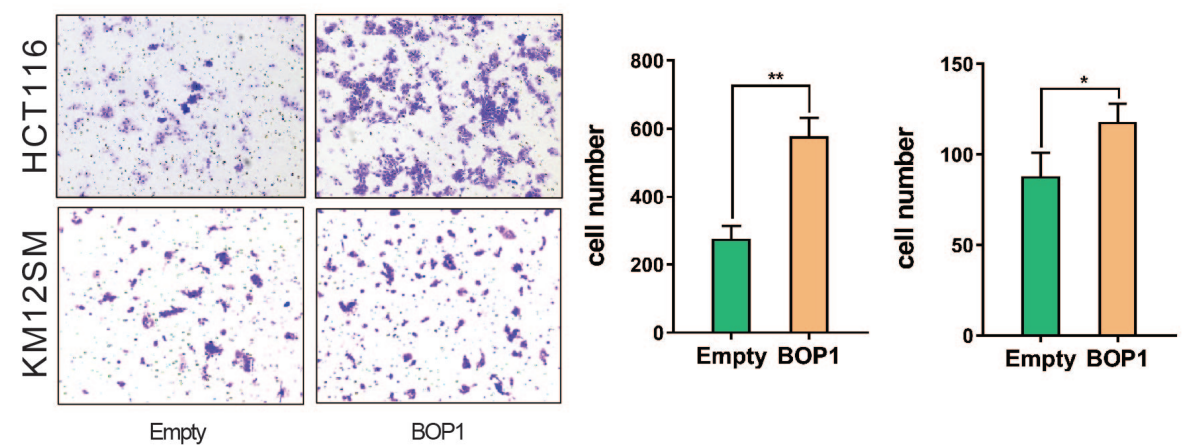


\section{Figure 6}
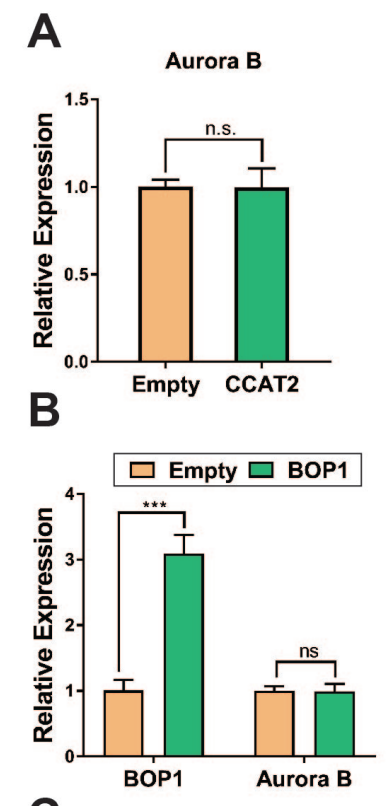

C

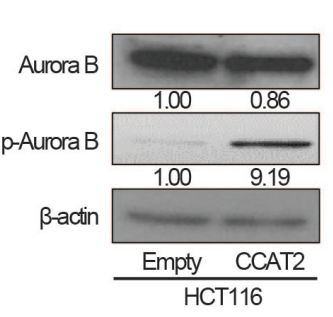

HCT116

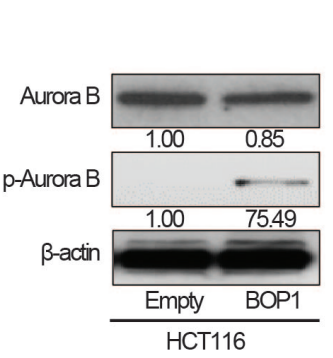

HCT116
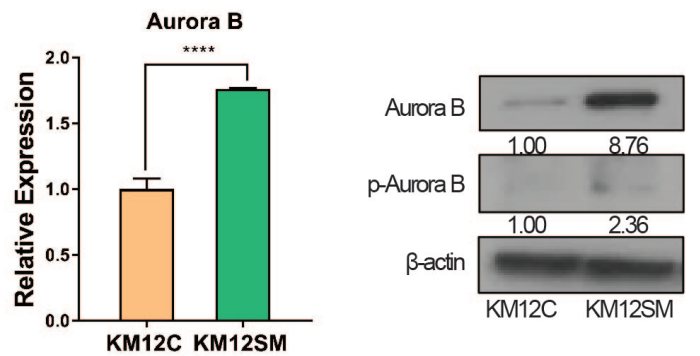

KM12SM

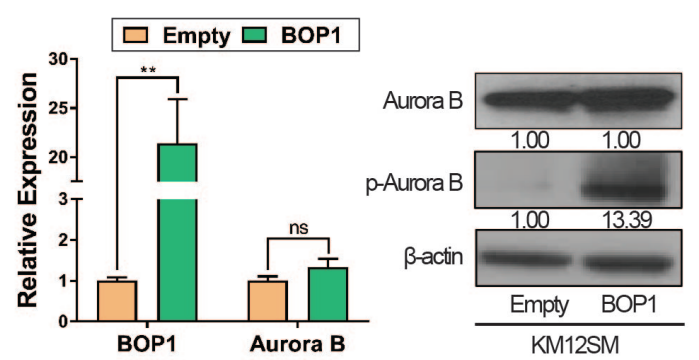

KM12SM

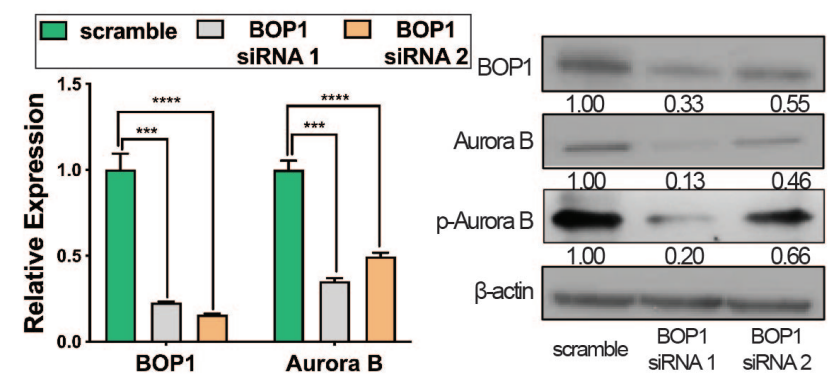

D
MYC tet-on system

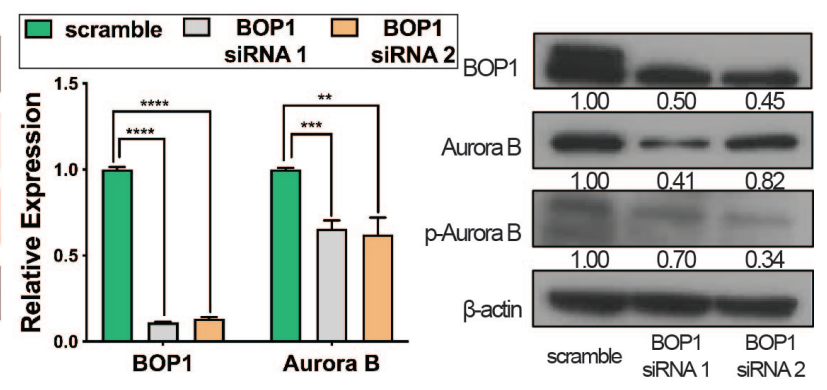

E

MS2-Pull down
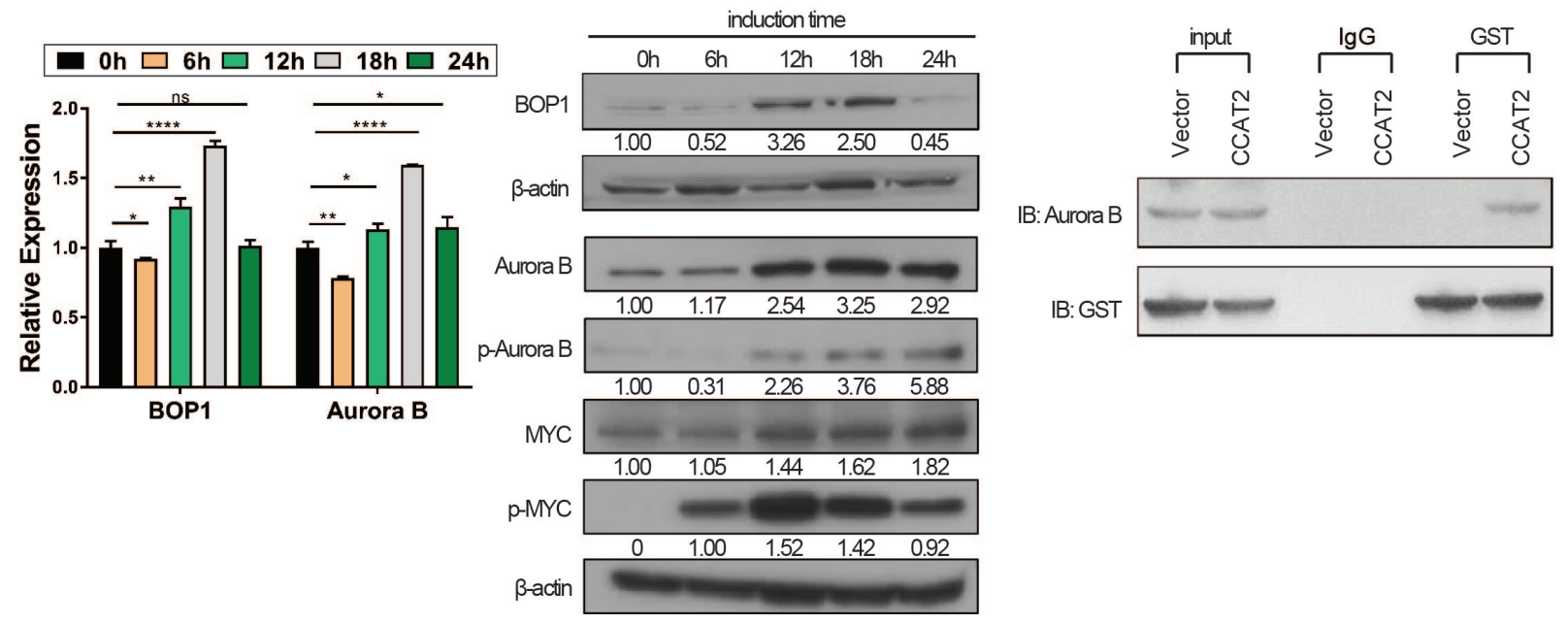
Figure 7

A

TCGA (Cohort A) Normal vs Tumor C CohortC Normal vs Tumor

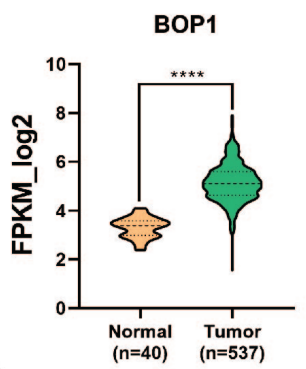

B
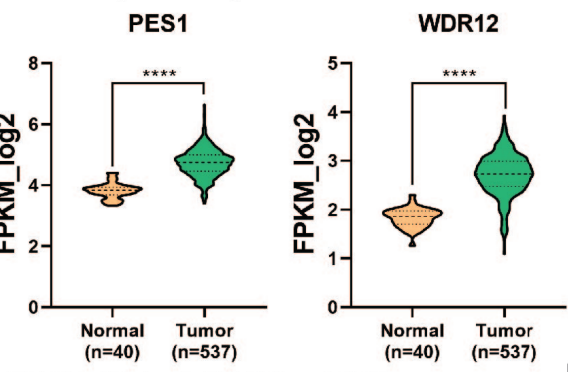

TCGA (Cohort A) MSI vs MSS

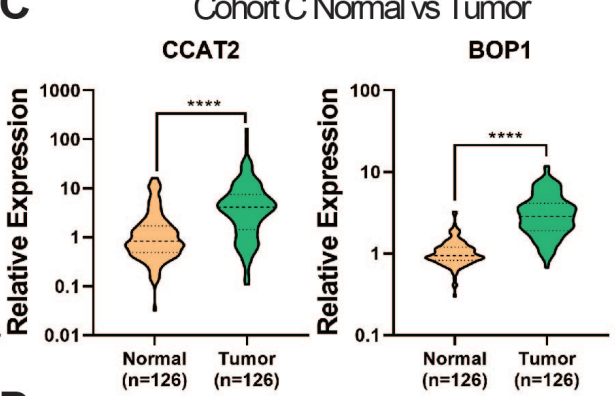

D CohortCMSI vs MSS
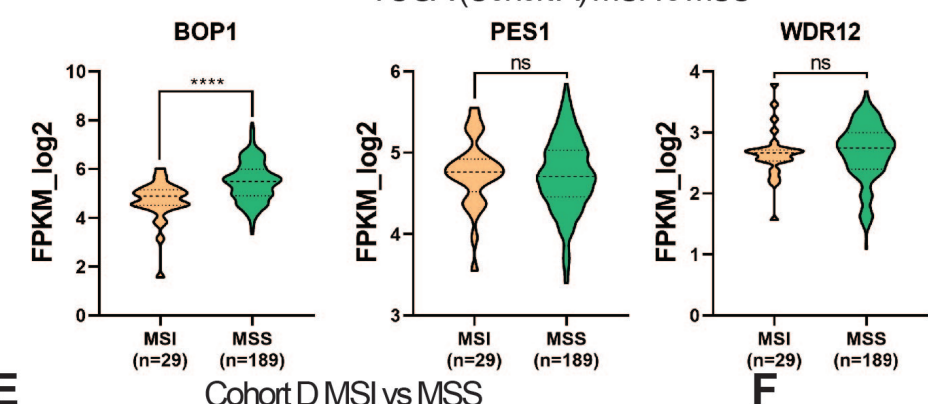

CCAT2

BOP1

E cсAT2
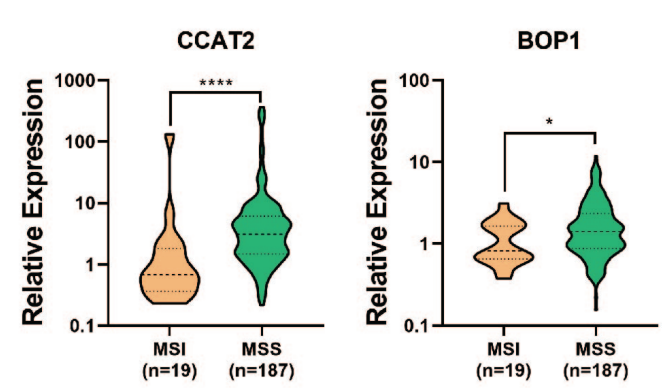

$\stackrel{(n=29)}{F}$
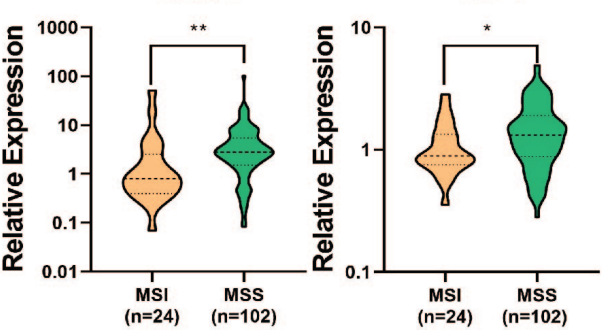

Tumor tissue

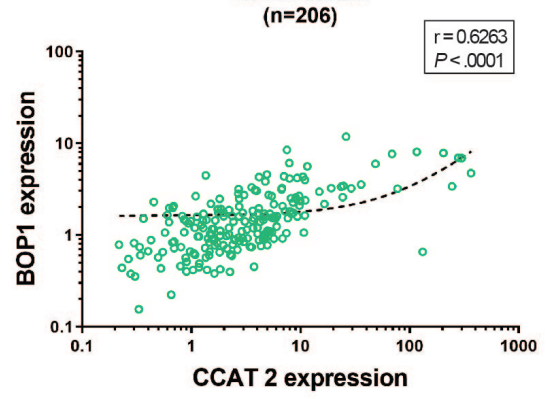

G

CohortD Overall sunvival
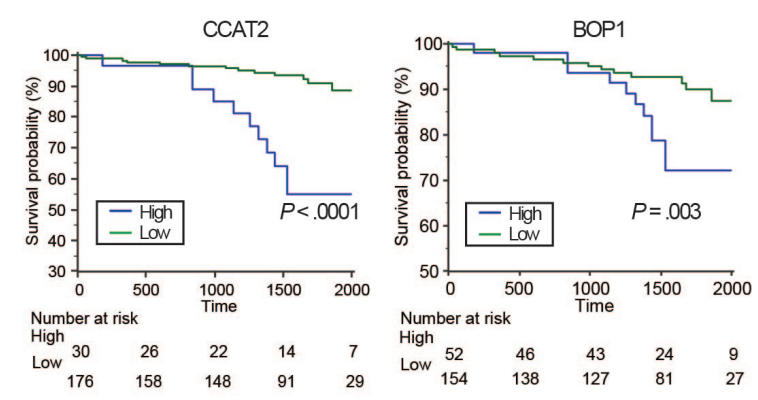

H CohortD Recurrence free survival
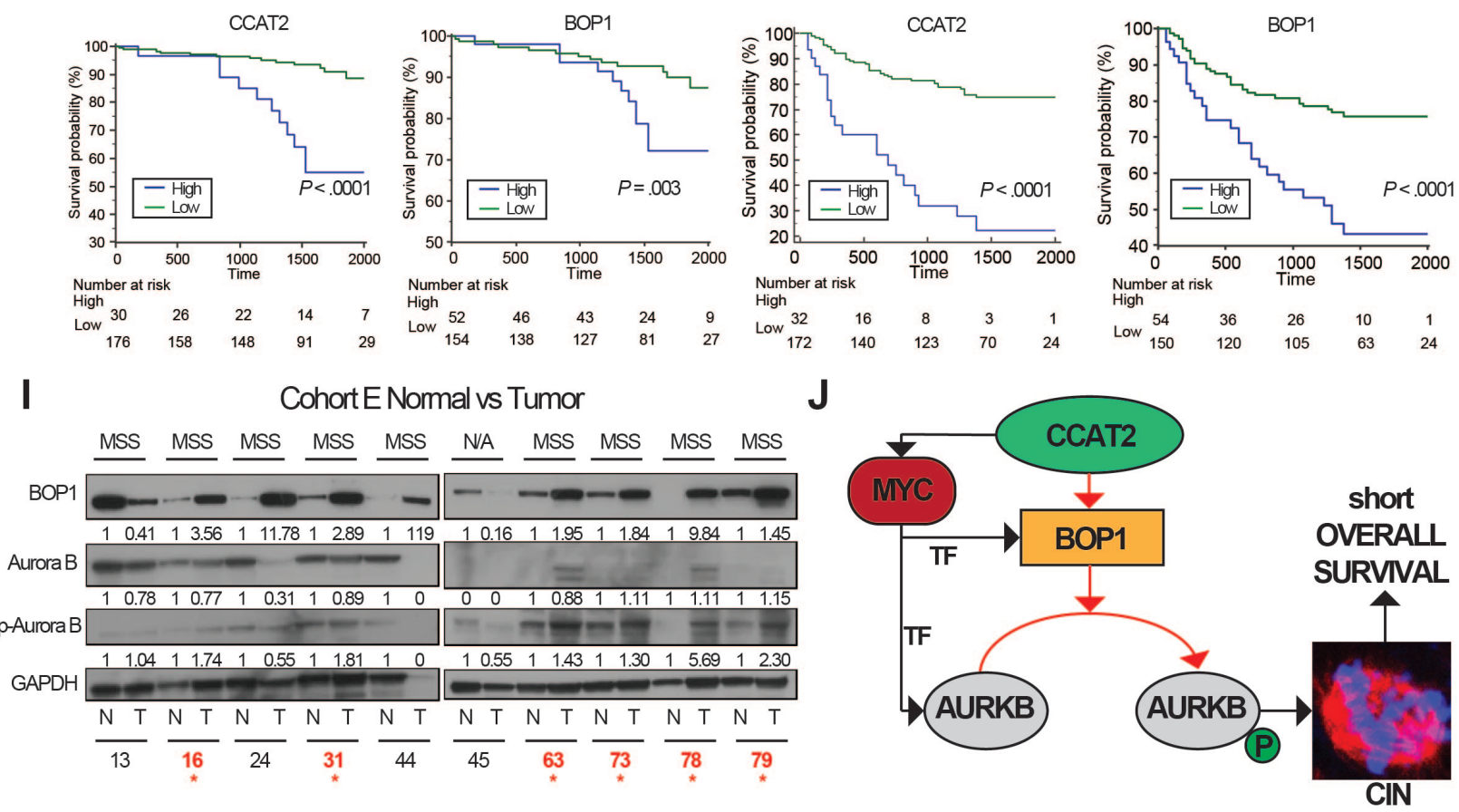



\section{What you need to know:}

Background and Context: High expression of the colon cancer associated transcript 2 gene (CCAT2), which encodes a long noncoding RNA (lncRNA), associates with chromosome instability, but little is known about how CCAT2 IncRNA regulates this cancer enabling characteristic.

New Findings: Colorectal cancer cells overexpress CCAT2 IncRNA, which promotes chromosome instability by stabilizing and inducing expression of BOP1 and activation of aurora kinase B.

Limitations: In this study pharmacological targeting of CCAT2 lncRNA was not assessed. Further studies are needed to analyze the targetability of CCAT2 IncRNA and associated toxicity.

Impact: Strategies to target this pathway might be developed for treatment of patients with microsatellite stable colorectal tumors.

Lay Summary: The authors identified a gene product that causes chromosomes to become unstable in colon cells, promoting development of colorectal cancer. 


\section{Supplementary materials and methods}

The Long Noncoding RNA CCAT2 induces chromosomal instability through BOP1 - AURKB signaling

Baoqing Chen, Mihnea P. Dragomir, Linda Fabris, Recep Bayraktar, Erik Knutsen, Xu Liu, Changyan Tang, Yongfeng Li, Tadanobu Shimura, Tina Catela Ivkovic, Mireia Cruz De los Santos, Simone Anfossi, Masayoshi Shimizu, Maitri Y. Shah, Hui Ling, Peng Shen, Asha S. Multani,

Barbara Pardini, Jared K. Burks, Hiroyuki Katayama, Lucas C. Reineke, Longfei Huo, Muddassir Syed, Shumei Song, Manuela Ferracin, Eiji Oki, Bastian Fromm, Cristina Ivan, Krithika Bhuvaneshwar, Yuriy Gusev, Koshi Mimori, David Menter, Subrata Sen, Takatoshi Matsuyama, Hiroyuki Uetake, Catalin Vasilescu, Scott Kopetz, Jan Parker-Thornburg, Ayumu Taguchi, Samir M. Hanash,

Leonard Girnita, Ondrej Slaby, Ajay Goel, Gabriele Varani, Mihai Gagea, Chunlai Li, Jaffer A. Ajani, George A. Calin

Supplementary Material and Methods

Supplementary figure 1-8

Supplementary tables 1-12

Supplementary References 


\section{Supplementary Material and Methods}

\section{Cell culture}

Human colon cancer cell lines HCT116, KM12C, KM12SM, COLO320, DLD-1, HT29 and gastric cancer cell lines AGS and KATO-III (Supplementary Table 7) were obtained from the American Type Culture Collection. Of note, KM12SM cell is the spontaneous liver metastasis of the KM12C cells ${ }^{1}$. HCT116 and HT29 cells were cultured in McCoy's 5A, COLO320, DLD-1, AGS and KATO-III in RPMI-1640, KM12C and KM12SM in DMEM with $4.5 \mathrm{~g} / \mathrm{L}$ glucose (10\% fetal bovine serum and $1 \%$ antibiotics) at $37^{\circ} \mathrm{C}$ with $5 \% \quad \mathrm{CO}_{2}$ and $95 \%$ humidity. All cell lines were validated by the Characterized Cell Line Core at The University of Texas MD Anderson Cancer Center using STR DNA fingerprinting.

\section{CCAT2 transgenic mice}

CCAT2 transgenic mice were generated as previously described ${ }^{2}$. The mice used for this study were both females and males of 7-9 months of age. Briefly, a human 1.7-kb cDNA of CCAT2 was cloned into a vector backbone which contained the CAG promoter, eGFP reporter gene and an IRES element. The CCAT2-vector was inserted randomly into the genome of C57BL/6N mice by pronuclear injection by the MDACC Genetically Engineered Mouse Facility. The founders were mated with WT C57BL/6N mice. Pups were selected for presence of the transgene by PCR on tailextracted DNA according to standard protocols. All the protocols and experiments were conducted according to the guidelines of the MDACC Institutional Animal Care and Use Committee.

\section{AOM/DSS mouse model}

Because of the sex-related differences in the chemically induced colorectal cancer model ${ }^{3}, 7-8$ week-old male mice of C57BL/6N strain 4-6, wild-type or CCAT2 transgenic, were used. Azoxymethane (AOM) was purchased from the Sigma-Aldrich (St Louis, MO). Colitis-grade Dextran Sodium Sulfate (DSS) with a molecular weight of 36000-50 000 was purchased from MP Biochemicals. All mice were initially treated with a single intraperitoneal injection of AOM (10 $\mathrm{mg} / \mathrm{kg}$ of body weight). Two days after AOM administration, the mice received 3\% DSS in their drinking water for 5 days, followed by a 2-week rest period without DSS. This scheme was repeated for a total of four DSS administrations. Mice were sacrificed one week after the last DSS administration. At this time, mice were dissected, the colon was excised and flushed with saline solution. The entire colon length from the cecum to rectum was cut open longitudinally exposing 
the mucosa for gross examination and taking pictures with Leica camera dissecting microscope for counting and measuring the mucosal polyps. Frozen tissue samples of grossly detected polyps and of normal colon mucosa were collected. Subsequently, the colon was fixed in $10 \%$ neutral buffered formalin for histopathological examination.

\section{Histopathologic Evaluation}

Two longitudinal serial sections of each colon were prepared histologically and examined microscopically by an ACVP certified veterinary pathologist. Histopathological evaluation included counting and size measurement of polyps (colon adenomas), and grading of hyperplastic and dysplastic changes of colonic glands with a score from 1 to 4 ( $1=$ minimal, $2=$ mild, $3=$ moderate, $4=$ marked). The entire colon from cecum to rectum was collected at necropsy and then was open longitudinally and gently flashed with PBS solution for cleaning the feces. The colon was attached to index-card paper for exposing the mucosa and photographing the entire colonic mucosa with the Leica stereomicroscope and camera for gross counting of the polyps. Then the colon tissue was immersed in $10 \%$ neutral buffered formalin for 48 hour fixation. Fixed colon tissues were arranged in "Swiss rolls" and cut longitudinally in 2 halves which were processed and embedded in paraffin blocks, from which $4-\mu \mathrm{m}$ thick sections were cut and stained with hematoxylin and eosin (H\&E). H\&E stained slides were scanned with Aperio AT2 scanner for microscopic examination and histomorphometric evaluation of colonic polyps.

\section{Murine colon crypts isolation and murine colon organoid culture}

Crypts were isolated and colon organoids were established as previously described ${ }^{7-9}$. Approximately $10 \mathrm{~cm}$ of colon was harvested from WT and CCAT2 transgenic mice, respectively. The colon was washed with cold PBS (without $\mathrm{Ca}^{2+}$ and $\mathrm{Mg}^{2+}$ ), opened laterally and cut into 2-mm pieces. The pieces were washed again several times with cold PBS with penicillin/streptomycin (Gibco, 15140122) until were clear. Then, tissue fragments were subjected to enzymatic digestion in $2 \mathrm{ml}$ digestion medium containing $2 \mathrm{mg} \mathrm{ml}^{-1}$ collagenase IV (Sigma-aldrich, C5138), $0.1 \mathrm{mg} \mathrm{ml}^{-1}$ dispase type II (Sigma-Aldrich, D4693), $10 \mu \mathrm{M}$ Y-27632 (StemCell, 72304), $100 \mathrm{U} / 100 \mu \mathrm{gl}^{-1}$ penicillin/streptomycin and 10\% FBS in DMEM medium (Gibco, 11995065), on an orbital shaker at room temperature for 30 minutes. The tissue mixture was then filtered through a $70-\mu \mathrm{m}$ cell strainer into a $50 \mathrm{ml}$ conical tube, and centrifuged at $200 \mathrm{xg}$ for five minutes at $4^{\circ} \mathrm{C}$. The pellets were resuspended in $10 \mathrm{ml}$ cold DMEM medium containing penicillin/streptomycin and FBS, and centrifuged again at $200 \mathrm{xg}$ for five minutes at $4^{\circ} \mathrm{C}$. 
Isolated crypts were counted using a hemacytometer with an inverted microscope, and embedded in growth factor-reduced Matrigel (Corning, catalog \# 356231), diluted 3:4 in organoid culture media and seeded into 24-well plates (Corning ${ }^{\mathrm{TM}} 3526$ ) at a density of 250-500 crypts in $50 \mu \mathrm{l}$ total volume per well, and overlaid with $500 \mu$ organoid culture media onto the Matrigel after incubation for 20 minutes at $37^{\circ} \mathrm{C}$.

Fresh medium was added every 2 or 3 days. Colon organoids were observed and treated at proper times. Outgrowing organoids were passaged every 7-days after mechanical and TrypLE ${ }^{\mathrm{TM}}$ Express (Gibco, 12604021) disruption. The organoids were washed several times with centrifugation at $200 \mathrm{~g}$ at $4{ }^{\circ} \mathrm{C}$. The pellets were suspended in Matrigel with a dilution ration 1:4 and seeded as described above.

Murine colon organoids were cultured in Advanced DMEM/F12 medium (Gibco, 12634010) containing 10 mM HEPES (Invitrogen, 15630-056), 1\% GlutaMAX (Invitrogen, 35050), 100 U/100 $\mu \mathrm{g} \mathrm{ml}^{-1}$ penicillin/streptomycin, supplemented with $50 \mathrm{ng} \mathrm{ml}^{-1}$ human EGF (PeproTech, 315-09), $200 \mathrm{ng} \mathrm{ml}^{-1}$ Noggin (Peprotech, 250-38), $500 \mathrm{ng} \mathrm{ml}^{-1} \mathrm{R}$-spondin (BioLegend, 783606), $1 \mathrm{mM} \mathrm{N}$ acetyl-L-cysteine (Sigma-Aldrich, A9165), 1× N2 (Gibco, 17502-048), 1× B27 (Gibco, 17504-044), $10 \mathrm{nM}$ gastrin (Sigma-Aldrich, G9145), $10 \mathrm{mM}$ Nicotinamide (Sigma-Aldrich, N0636), $10 \mu \mathrm{M}$ Chiron (Sigma-Aldrich, SML1046), 500 nM A83-01 (StemCell, 72024), 10 M SB202190 (SigmaAldrich, S7067), $10 \mu \mathrm{M}$ Y-27632, $10 \mathrm{nM}$ prostaglandin E2 (Selleck Chemicals, S3003), and 1× Primocin (InvivoGen, ant-pm-1)]. Organoids cell viability was measured every three days after treatment by using CellTiter-Glo ${ }^{\circledR}$ 3D Cell viability assay (Promega, G9683) according to manufacturer's instruction.

\section{Gastric cancer PDX and PDO}

GA-080417 cell line (referred to as \#1) was established directly from patient ascites by culturing the ascites cells in RPMI (7\% FBS, 1\% antibiotics) and expanding them for more than 10 passages. This cell line was then injected to SCID mice by both subcutaneous $\left(1 \times 10^{4} \sim 1 \times 10^{5}\right)$ and orthotopic $\left(1 \times 10^{5}\right)$ administration to investigate their tumorigenicity. GA-080417 cells can grow into tumors after both subcutaneous and orthotopic injection.

In contrast, GA-082517 cell line (referred to as \#3) was established from PDX tumor that was generated by subcutaneous injection of GA-082517 patient ascites cells. Tumor cells were disassociated from PDX tumor tissue and cultured in RPMI (7\% FBS, 1\% antibiotics) followed by 
about 10 passages. The GA-082517 cell line was tested for tumorigenicity by both subcutaneous $\left(1 \times 10^{3} \sim 1 \times 10^{5}\right)$ and orthotopic $\left(1 \times 10^{5}\right)$ administration in SCID mice and was found to have tumor growth through both administration routes.

GA-051816 cell line (referred to as \#2) was previously reported, for more detail refer to Song et al. ${ }^{10}$.

\section{Colon cancer PDX}

Colon cancer PDX were established as previously described ${ }^{11}$. About 6-8-weeks old female NOD.Cg-Prkdcscid Il2rgtm1Wjl/SzJ (NSG) mice were maintained in the MDACC animal facilities following standard animal regulation and strict health control. Rodent care and housing were in accordance with institutional guidelines and regulations as well as according to Institutional Animal Care and Use Committee approved animal protocols. Patient tumor (PX) specimens acquired at MDACC were engrafted into NSG mice. One tumor fragment ( $\left.\sim 50 \mathrm{~mm}^{3}\right)$ per mouse was implanted subcutaneously into the flanks of mice anesthetized under 2-4\% isoflurane $/ \mathrm{O}_{2}$ inhalation. Primary tumor xenografts (P0) growth was monitored and documented twice a week, with the date of first palpable growth noted. When tumor burden reached $1500 \mathrm{~mm}^{3}$, mice were euthanized for tumor collection. Sections of these tumors were transplanted into new mice for PDX establishment (P1). Tumor samples at each passage were collected for histology, protein, and genomic analysis. Several PDX samples were collected in freezing media (CryoStor CS10) for storage in liquid nitrogen.

\section{Patient sample collection}

Cohort A (TCGA colorectal cancer cohort) consisted of 537 CRC cases with clinical and mRNA expression information. Among them 40 had mRNA data for matched normal tissues. The clinical information was retrieved from ${ }^{12}$. The information regarding the MSS/MSI status for 275 patients was obtained from the Cancer Genome Atlas ${ }^{13}$. The clinical characteristics were listed in Supplementary Table 1. For this cohort of patients, fragments per kilobase millions (FPKM) quantification mRNA-seq data from the Genomic Data Commons Data Portal (https://portal.gdc.cancer.gov/) for BOP1, PES1 and WDR12 genes were downloaded and log2 transformed.

For Cohort B, RNA samples from frozen cancer and matched non-neoplastic tissues of resected specimen from 100 CRC patients were obtained from the Department of Surgery and Science 
(Department of Surgery II), Kyushu University Hospital. Non-neoplastic tissues were obtained from the resected specimen and were sufficiently far enough from the primary tumor. Histological diagnosis was made according to the World Health Organization criteria and pathological staging was done in accordance to the tumor-node-metastasis (TNM) classification system. Cases with stage IV were patients with synchronous distant metastasis when undergoing surgery. The details of the patient's clinical characteristics were listed in the Supplementary Table 2.

Cohort $\mathbf{C}$ consisted of RNA samples isolated from tumor and adjacent normal colon tissue samples of a total of 126 CRC patients from the Department of Comprehensive Cancer Care, Masaryk Memorial Cancer Institute, Czech Republic. Cohort C was composed of 24 MSI-H and 102 MSS (MSS/MSI-L) sporadic CRC patients. pTNM classification of the patients was done based on pathology reports and histological slides (Supplementary Table 3). The patients underwent standard surgical procedure and adjuvant therapy was added when necessary (stage II with risk factors or stage III). In case of advanced disease at the time of diagnosis, patients received adjuvant treatment according to the oncologist choice, following the recommendations of national guidelines.

In the Cohort D, a total of 206 fresh frozen tissue specimens, which encompassed primary colorectal adenocarcinoma tissues from 19 MSI-H and 187 MSS (MSI-L and MSS) cancer patients, were collected from Tokyo Medical and Dental University, Japan. Patients undergoing resection of their primary tumor that was histologically confirmed to be a stage II and III CRC and classified as MSI-H or MSS (MSI-L and MSS) were included in this study. Details of the clinical and pathological features of the included patients are shown in Supplementary Table 4.

Cohort $\mathbf{E}$ consisted of 10 paired samples, normal colon mucosa and colon tumor. The samples were obtained from the Ruder Boskovic Institute, Croatia. Tissue samples were obtained from fresh surgical specimens frozen in liquid nitrogen and stored at $-80^{\circ} \mathrm{C}$. The samples were histologically confirmed prior to use. Nine out of ten samples were classified as MSS and for one sample the status was not available (N/A). The CCAT2 expression for this cohort was previously reported ${ }^{14}$.

\section{CIN index (CINdex) analysis}

Segmented copy number data (as on hg19 reference genome), along with RNA-seq gene expression counts from TCGA Colon Adenocarcinoma (COAD) and TCGA Stomach Adenocarcinoma 
(STAD) patients were downloaded from the Broad Institute Firebrowse platform (http://firebrowse.org/). An intersection was done to select only those 280 COAD tumor samples that had both copy number and gene expression data. An intersection was done to select only those 412 STAD tumor samples that had both copy number and gene expression data.

Bioconductor package CINdex (http://bioconductor.org/packages/CINdex/) ${ }^{15}$ was applied on the segmented copy number data which enabled to characterize genome-wide DNA copy number alterations as a measure of chromosomal instability. This package calculated genomic instability at the chromosome and cytoband level. Only data from autosomes were considered for this analysis. A threshold of 2.25 and 1.75 in the un-normalized setting was used to define gains and losses respectively.

The chromosome CIN and gene expression data from 280 COAD and 412 STAD patients were shifted by 1 , respectively 2 and then converted to the log base 2 scale. A Pearson correlation analysis was performed between the chromosome CIN and the gene expression data and results with a p-value less than 0.05 were short listed for further inspection. A customized correlogram table was plotted where each cell represented a correlation between two variables. Positive correlation was represented by orange color while negative correlation represented by purple colors. The cells were colored white if $\mathrm{p}$-value of a correlation test was not significantly different from zero. All analyses were performed using the $\mathrm{R}$ statistical platform (https://www.rproject.org/).

\section{Genomic instability analysis}

Chromosome analyses were performed as previously reported ${ }^{2}$. Cells/mouse organoids were plated into $10 \mathrm{~mm}$ plates. After reaching $60-70 \%$ confluence, cells were first exposed to colcemid $(0.04 \mathrm{mg} / \mathrm{mL})$ for $1-2$ hours at $37^{\circ} \mathrm{C}$ and to hypotonic treatment $(0.075 \mathrm{M} \mathrm{KCl})$ for $20 \mathrm{~min}$ at room temperature. Afterwards, cells were fixed in a methanol and acetic acid mixture (3:1 by volume) for $15 \mathrm{~min}$ and washed three times with the fixative. Air-dried preparations were made and the slides were stained with $4 \%$ Giemsa. The slides were analyzed for chromosomal aberrations, including chromosome and chromatid breaks, fusions, fragments and tetraploidy. A minimum of 35 metaphases were analyzed from each sample. Images were captured using a Nikon 80i microscope equipped with karyotyping software from Applied Spectral Imaging, Inc. Carlsbad, CA, USA. 


\section{Immunofluorescence staining}

Cells were cultured in 8-well chamber slides (Ibidi). To check the aberrant spindles, cells were synchronized by culturing in the medium with nocodazole $(100 \mathrm{ng} / \mathrm{ml})$ for 14 hours and then were released to process to $\mathrm{G} 2 / \mathrm{M}$ phase by removal of nocodazole. Cells were fixed with $4 \%$ paraformaldehyde for $30 \mathrm{~min}$ at room temperature and then washed with PBS for three times, followed by blocking and permeabilization with blocking buffer, which includes 4\% BSA and 0.3\% Triton X-100, for 1 hour at room temperature. For the analysis of the spindle apparatus, cells were incubated overnight at $4^{\circ} \mathrm{C}$ with mouse anti- $\alpha$-tubulin $(1: 16000$; CST) and then with anti-mouse secondary antibodies (Alexa Fluor 647; Invitrogen) for 1 hour at room temperature. Slides were finally mounted with mounting medium containing DAPI (Abcam). For analyzing the anaphase bridges, cells were cultured, fixed, blocked, and permeabilized with same procedure and reagents. Then, the cells were stained with DAPI (Abcam 1:5000) directly, and washed three times with PBS to remove the background signal. Images were acquired with the Spin Disc Confocal microscope (Andor).

\section{Plasmids and constructs}

Mammalian expression vectors for full-length CCAT2 and a series of mutants were constructed by subcloning the gene sequences into pCDNA3.1 (+) backbone (Life Technologies), pBabe retroviral expression vector, or MS2-24x-pCNDA vector. The full-length FLAG-tagged BOP1 human expression vector was purchased from Origene (\#RC204016), and GST-tagged recombinant human BOP1 protein was purchased from Novus Biologicals. The BOP1 and CCAT2 truncated mutants were generated by using QuikChange II XL Site-Direct Mutagenesis Kit (Agilent Technologies, \#200522). All constructs were confirmed by DNA sequencing at Sequencing and Microarray Facility (SMF), UT MD Anderson Cancer Center.

\section{RNA interference}

CCAT2, BOP1, or negative control siRNAs were purchased from Ambion. Cells were seeded into six-well plates. When the cells reached $50-70 \%$ confluence, they were transfected with $25 \mathrm{nM}$ of the corresponding siRNA by Lipofectamine 2000 (Life Technologies) according to the manufacturer's protocol. RNA and proteins were collected at $48 \mathrm{~h}$ (in the BOP1 knock-down experiments) or $72 \mathrm{~h}$ (in the CCAT2 knock-down experiments) after transfection. qRT-PCR and Western blot were used to check the efficiency of knock-down. 


\section{Generation of stable clones}

HCT116 cells with CCAT2 stable overexpression (HCT116CCAT2) were established by transfecting pcDNA 3.1 CCAT2-expression vector with Lipofectamine 2000 (Invitrogen, ThermoFisher Scientific) as previously described 16. Empty clone was generated by transfection with the empty pcDNA3.1 vector (HCT116 Empty). In our previous papers 14,16 these clones were termed: empty (E) and overexpressed clone 1 (OC1). DLD-1Empty and DLD-1 ${ }^{\text {CCAT2 }}$ were established using the same above-mentioned method.

The cell culture supernatants with lentivirus carrying BOP1 were purchased from the MDACC shRNA and ORFeome Core of The University of Texas MD Anderson Cancer Center. HCT116, KM12SM, and HT29 cells were plated into six-well plates. Cells were infected by the cell-free supernatants containing lentivirus with $10 \mu \mathrm{g} / \mu \mathrm{L}$ polybrene (Sigma) for 48 hours and then switched to normal medium with Blasticidin S for 72 hours. Cells with successful transduction showed green fluorescence. Flow cytometry was used to select the green fluorescence-positive cells. We termed the clones with BOP1 overexpression HCT116 ${ }^{\mathrm{BOP} 1}$, KM12SMBOP1, and HT29BOP1.

For MYC tet-on stable clones, we first generated the HCT116 tetracycline reverse transcriptional activator (rtTA) stable clones by transducing with CMV-rtTA lentivirus and selected by neomycin (Sigma), as the activation of genes downstream of Tet0 induced by doxycycline is rtTA dependent. Then the stable HCT116 rtTA clones were transduced with tet-on C-MYC lentivirus and selected by puromycin (Sigma). CMV-rtTA lentivirus and inducible tet-on C-MYC lentivirus were purchased from Cellomics Technology.

\section{RNA extraction, cDNA synthesis and quantitative real-time PCR}

Total RNA was isolated using Direct-zol kit (Zymo research) following the manufacturer's protocol. Then, the cDNA was synthesized using High-Capacity cDNA Reverse Transcription Kit (Thermo Fisher Scientific) according to the manufacturer's protocol. qRT-PCR was performed using SsoAdvanced ${ }^{\mathrm{TM}}$ Universal SYBR Green Supermix real-time PCR kit (Bio-Rad). Primers were synthesized by Integrated DNA Technologies (sequences are listed in Supplementary Table 5). The relative gene expression levels were calculated using the $2^{-\Delta \Delta \mathrm{Ct}}$ method. The geometric mean of GAPDH, $\beta$-actin, and U6 snRNA were used as normalizer for in vitro studies; mAactb and Rplpo mRNAs were used as housekeeping genes for in vivo experiments while for clinical samples we used $\beta$-actin or geometric mean of GAPDH, $\beta$-actin, and U6 snRNA as normalizer. The absolute RNA 
expression of the house keeping genes showed minimal and non-statistical difference between the groups we compared.

\section{Whole or cytosolic/nuclear protein fractionation and Western blot}

Protein lysates from whole-cell pellets samples were generated using Cell Lysis Buffer (Cell Signaling Technology) that contains protease and phosphatase inhibitor cocktails (Sigma-Aldrich). Cytosolic and nuclear protein fractionations were performed by utilizing of the NER Nuclear and Cytoplasmic Extraction Reagents kit (ThermoFisher Scientific) according to the manufacturer's protocol. Protein concentration was quantified by Bradford assay (Bio-Rad). In total, 20 ug of proteins were loaded on 4-20\% acrylamide CriterionTM TGXTM precast gels (Bio-Rad) and transferred to nitrocellulose membranes by semi-dry method. The membranes were incubated with the corresponding primary antibodies (listed in Supplementary Table 6) overnight and then incubated with the appropriate HRP-conjugated secondary antibody. Immunoreactivity was detected by incubation with ECL SuperSignal West Femto substrate (ThermoFisher Scientific), and then detected by the autoradiographic film.

\section{Cytosolic and nuclear RNA fractionation}

RNA was isolated from cellular fractions according to the previously described protocol 17. Briefly, the cell pellet was resuspended in 380 ul Hypotonic lysis buffer (HLB: 10 mM Tris (pH 7.5), $10 \mathrm{mM} \mathrm{NaCl}, 3 \mathrm{mM} \mathrm{MgCl} 2,0.3 \%$ (vol/vol) NP-40 and 10\% (vol/vol) glycerol) and the mixture was incubated on ice for $10 \mathrm{~min}$. The cells were centrifuged at $1,000 \mathrm{x} \mathrm{g}$ at $4{ }^{\circ} \mathrm{C}$ for $3 \mathrm{~min}$; the supernatant, which is the cytoplasmic fraction, was transferred to another tube. RNA precipitation solution (RPS: $0.5 \mathrm{ml}$ of $3 \mathrm{M}$ sodium acetate (pH 5.5) with $9.5 \mathrm{ml}$ of ethanol) was added and the mixture was stored at $-20{ }^{\circ} \mathrm{C}$ for over $1 \mathrm{~h}$. The remaining pellet (the nuclear fraction) was washed three times with HLB by centrifuging at $200 \mathrm{x}$ g at $4^{\circ} \mathrm{C}$ for $2 \mathrm{~min}$. TRIzol was added over the nuclear pellet and RNA was extracted using Direct-zol kit (Zymo research) following the manufacturer's protocol. The cytoplasmic fraction, after $1 \mathrm{~h}$, was centrifuged at $18,000 \mathrm{x} \mathrm{g}$ at $4{ }^{\circ} \mathrm{C}$ for $15 \mathrm{~min}$. The pellet was washed in $70 \%$ ethanol and centrifuged again at $18,000 \mathrm{x}$ g at $4{ }^{\circ} \mathrm{C}$ for 5 min. After air drying, $1 \mathrm{ml}$ of TRIzol was added to the pellet and RNA was extracted. The lncRNA NEAT1 was used as a positive control for nuclear enrichment.

\section{$\gamma-\mathrm{H} 2 \mathrm{AX}$ assay}


This assay was performed with and without Bleomycin treatment. HCT116 Empty and HCT116 ${ }^{\text {CCAT2 }}$ overexpressed cells were cultured for 24 hours in 8-well chamber slides (Ibidi). In the case of Bleomycin treatment, $10 \mu \mathrm{g} / \mathrm{ml}$ of Bleomycin was added for two hours at $37^{\circ} \mathrm{C}$. Treated and untreated cells were then washed three times with PBS and fixed by incubating them in ice cold methanol for 5 minutes at room temperature. This step was followed by blocking and permeabilization with $1 \%$ BSA-PBST at $37^{\circ} \mathrm{C}$ for 30 minutes. Next, the cells were incubated overnight at $4{ }^{\circ} \mathrm{C}$ with the primary antibody of $\mathrm{H} 2 \mathrm{AX}$ (rabbit polyclonal IgG) followed by one-hour incubation at room temperature with secondary antibody, goat polyclonal IgG conjugated with FITC. Finally, cells were stained with Hoechst 33342, before adding the cover slip. Images were acquired with the Spin Disc Confocal microscope (Andor).

\section{Cellular Senescence Staining}

HCT116 Empty and HCT116 ${ }^{\text {CCAT2 }}$ cells were seeded in 6-well plates to a seeding density of $3 \times 10^{5}$ cells per $\mathrm{mL}$, and a final chamber volume of $1 \mathrm{~mL}$. When the cells reached $70-80 \%$ confluence, were stained for senescence using a $\beta$-Galactosidase Staining Kit (Cell Signaling Technology \#9860) following the manufactures instructions. Cells were mounted for analysis with $70 \%$ glycerol. All the cells from the circumference of three wells were analyzed for each clone.

\section{SHAPE Analysis}

RNA secondary structure probing was started by mixing stock CCAT2 RNA and folding buffer (200 mM NaCl, $100 \mathrm{mM}$ HEPES, $0.2 \mathrm{mM}$ EDTA, pH 8.0), followed by incubation at $37^{\circ} \mathrm{C}$ with refolding buffer for $30 \mathrm{~min}$ (100 mM NaCl, $50 \mathrm{mM}$ HEPES, $16.5 \mathrm{mM} \mathrm{MgCl2,} \mathrm{pH} \mathrm{8.0).} \mathrm{The} \mathrm{SHAPE}$ reaction was then started by adding NMIA $(+)(32.5 \mathrm{mM}, 65 \mathrm{mM}$ or $130 \mathrm{mM})$, or DMSO as control $(-)$. Samples were then incubated for $45 \mathrm{~min}$ at $37^{\circ} \mathrm{C}$, precipitated with ethanol and glycogen. Reverse transcription was performed using Super Script III reverse transcriptase (Invitrogen). Electrophoresis on an $8 \%$ (vol/vol) polyacrylamide gel was then performed to separate fragments. Band-intensities were visualized by gel electrophoresis or capillary electrophoresis and were quantified using SAFA, version 1.1 Semi-Automated Footprinting Analysis ${ }^{18}$. SHAPE reactivity data from capillary and gel electrophoresis were incorporated as a SHAPE constraint file in the RNA structure folding program, and the 20 lowest energy structures based on those constraints were generated 19, 20. Structures were calculated with RNAstructure default secondary structure options. Each structure image presented in this manuscript was rendered using VARNA. 


\section{Cycloheximide Chase Assay}

Protein half-life studies were performed as previously described ${ }^{21}$. Cells were cultured in sixwell plates and then transfected with CCAT2 or empty vector for 24 hours and then cultured with cycloheximide (CHX, 100 $\mathrm{g} / \mathrm{ml}$ ), which blocks the translation of mRNA. After culturing with cycloheximide for $0,4,8,12$, and 20 hours, the cells were collected and lysed with lysis buffer (CST) for protein extraction. The degradation of BOP1 was then detected by Western blotting analysis.

\section{Proliferation assay}

Cell Counting Kit-8 (Enzo) was used to determine the cell viability according to the manufacturer's protocol. Cells were seeded at a density of 2500 cells per well in 96-well plates overnight. For the proliferation assay in knock-down experiments, cells were transfected with the corresponding siRNA and then seeded in 96-well plates. After culturing for 0, 24, 48 and 72 hours, 10ul of CCK8 was added to each well and then incubated at $37^{\circ} \mathrm{C}$ for 2 hours. The absorbance values at $450 \mathrm{~nm}$ were measured to represent the cell viability.

\section{MTT assay - 5- Fluorouracil.}

In vitro chemoresistance to 5-Fluorouracil (5-FU) of HCT116 CCAT2 clones versus HCT116 Empty was assessed by MTT. Briefly, cells were plated 24 hours prior to treatment in 96-well microculture plates. After 24 hours, 3 different doses of 5-FU were added (2, 20 and $40 \mu \mathrm{M})$ to the supernatant without changing the medium. After 48 hours, the MTT reagent (Sigma) was added to each well and incubated for 3 hours at $37^{\circ} \mathrm{C}$. The optical density (OD) was read at $570 \mathrm{~nm}$ on a microplate spectrophotometer and growth values (\%) were calculated as followed (OD treated cells /OD untreated cells) x 100.

\section{MTS assay - oxaliplatin}

Cell viability of oxaliplatin treated HCT116 CCAT2 clones versus HCT116 Empty cells was measured by The CellTiter $96^{\circledR}$ AQueous One Solution Cell Proliferation Assay (Promega). Cells were seeded in $100 \mu \mathrm{l}$ medium into 96 -well plates and incubated for 24 hours at $37^{\circ} \mathrm{C}$ before treatment. The cells were treated with different concentrations of oxaliplatin: 2, 20 and $40 \mu \mathrm{M}$ (Selleckchem) for 48h. At the end of treatment, $20 \mu \mathrm{l}$ of MTS solution was added to each well of the plate and the optical density (OD) at $490 \mathrm{~nm}$ was then measured using microplate reader and growth values (\%) were calculated as followed (OD treated cells /OD untreated cells) x 100. 


\section{Colony formation assay}

Around 500 HCT116 cells with BOP1 knock-down or overexpression and KM12SM cells with BOP1 overexpression were plated into 6-well or 12-well plates. For the knock-down experiments models, corresponding siRNAs were added and then incubated for 48 to 72 hours. Cells were then cultured with new medium for 10-14 days. The colonies were fixed with $4 \%$ paraformaldehyde solution, stained with crystal violet. Colonies with more than 50 cells were counted.

\section{Invasion and scratch assay}

Cells in serum-free media were seeded into precoated Matrigel 24-well invasion chambers (8 mm; 24 wells; BD Biosciences) according to the manufacturer's protocol. In the bottom well, 700 $\mu \mathrm{l}$ of medium with $20 \%$ FBS was added to serve as chemoattractant. Twenty-four hours after seeding, cells at the top of the insert well were removed by swabbing, the cells that penetrated the membrane and were located on the bottom side of the insert well were fixed with paraformaldehyde, stained with crystal violet, and counted. For scratch assay, cells were seeded in 6-well plates and cultured to reach around 90-100\% confluency. A scratch was made by scratching a line across the bottom of each well using a $20 \mu \mathrm{L}$ pipette tip. The detached cells were then removed by washing with PBS. Migration into the open area was recorded at 48 hours after scratching.

\section{Polysome profiling}

Polysome profiling was done in accordance with previously published protocols ${ }^{22,23}$. Briefly, $10^{6}$ cells were treated 10 minutes at $37^{\circ} \mathrm{C}$ with cycloheximide at $100 \mathrm{ug} / \mathrm{ml}$. Cells were then trypsinized and collected under ice cold conditions. Cell lysates were prepared using dounce homogenization with 50 strokes of the dounce. The lysate was then precleared at $1200 \mathrm{xg}$ for 10 minutes, and equal OD254 units were loaded onto a $17-50 \%$ sucrose gradient. The sucrose gradients were fractionated on a gradient fractionator using a UA-6 detection system (Teledyne ISCO). For experiments to quantify absolute amounts of $40 \mathrm{~S}$ and $60 \mathrm{~S}$ subunits, a modified procedure was followed essentially as described ${ }^{24}$. In this procedure, the lysis buffer consisted of

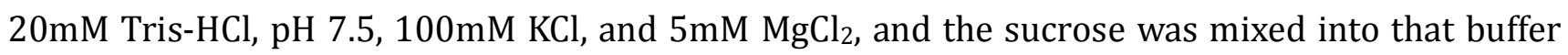
for pouring the sucrose gradients. Lysates were precleared as described above, and EDTA was added to a final concentration of $50 \mathrm{mM}$ prior to fractionating.

\section{In vitro RNA-protein binding assay}


Biotin-labeled full-length and truncated fragments of CCAT2 RNA were transcribed in vitro with a Biotin RNA Labeling Mix Kit (Roche) and T7 or SP6 RNA polymerase (Ambion) using the PCR products as a template, treated with RNase-free DNase I (Ambion). The reaction mix was then purified with an RNA Clean \& Concentrator kit (Zymo Research) and the purified biotinylated RNA was denatured at $95^{\circ} \mathrm{C}$ for $2 \mathrm{~min}$, cooled on ice for $2 \mathrm{~min}$, and then transferred in RNA structure/folding buffer at $30^{\circ} \mathrm{C}$ for 30 min to allow proper RNA secondary structure formation.

The RNA-protein binding assays were performed using Pierce ${ }^{\mathrm{TM}}$ Magnetic RNA-Protein PullDown Kit (Thermo Scientific) according to the manufacturer's instructions. Briefly, magnetic Dynabeads M-280 Streptavidin beads were washed three times with washing buffer and then immediately subjected to capture in vitro transcribed RNA ( $2 \mu \mathrm{g})$ as described above. To pull down recombinant proteins, the RNA-captured beads were incubated with recombinant proteins $(1 \mu \mathrm{g})$ in binding buffer for 1 hour at RT. Beads were washed three times and boiled in $1 \mathrm{x}$ reducing sample buffer. The retrieved proteins were analyzed by Western blotting.

\section{MS2 pull-down assay}

The pCCAT2-MS2, pEmpty-MS2, and pMS2-GST vectors were generated as previously reported 14. HCT116 cells were plated into $15 \mathrm{~mm}$ plates. After reaching $60-70 \%$ confluence, the cells were co-transfected with pCCAT2-MS2 vector or pEmpty-MS2, and pMS2-GST vector using Lipofectamine 2000 (Invitrogen, ThermoFisher Scientific). Forty-eight hours after transfection, cells were harvested and proteins were collected and quantified. In total, $1000 \mu \mathrm{g}(2 \mu \mathrm{g} / \mu \mathrm{l})$ lysate was incubated with GSH agarose beads (GE Healthcare) for 3 hours at $4^{\circ} \mathrm{C}$, followed by 3 times washing with cell-lysis buffer to remove unspecific bound proteins. Beads were then suspended in SDS buffer and heated. Bound proteins were detected by Western blotting.

\section{RNA immunoprecipitation}

EZ-Magna RIP RNA-Binding Protein Immunoprecipitation Kit (Merck Millipore) was used according to the manufacturer's protocol. Cells were harvested and lysed in the RIP buffer containing the protease inhibitor. Cell lysates were then incubated with the buffer containing magnetic beads conjugated with anti-BOP1 (Abcam), anti-PES1 (Santa Cruz), anti-WDR12 (Abcam), anti-AURKB or IgG as a negative control at $4^{\circ} \mathrm{C}$ overnight and then washed with washing buffer for three times to remove the unspecific bounds. RNA was then isolated using the phenol:chloroform:isoamyl alcohol method and further used for cDNA synthesis and qRT-PCR to 
test the presence of CCAT2. For CCAT2 segments - Aurora Kinase B interaction, UV crosslinked cells were used. The lysates were treated with RNase I for exactly $3 \mathrm{~min}$ at $37^{\circ} \mathrm{C}$ then immediately transferred on ice and centrifuged. The supernatant was incubated with magnetic beads conjugated with anti-AURKB overnight. After immunoprecipitation, RNA was purified with phenol:chloroform:isoamyl alcohol to prepare cDNA. Then, CCAT2 segment specific primers were applied to perform qRT-PCR.

\section{Gene expression analysis (GEA)}

For the mouse GEA, total RNA was extracted from bone marrow (BM) cells of WT and CCAT2 transgenic mice. Labeling and hybridization of mRNAs were performed according to Affymetrix protocols. Briefly, $5 \mu \mathrm{g}$ of total RNA was reverse transcribed with an oligo(dT) primer that has a T7 RNA polymerase promoter at the 5' end. Second-strand synthesis was followed by cRNA production with incorporation of biotinylated ribonucleotides using the BioArray High Yield RNA Transcript Labeling Kit T3 (Enzo Life Sciences). The labeled cRNA was fragmented and hybridized to Affymetrix GeneChip Mouse Genome 2304.0 arrays. GeneSpring GX software v.13 (Agilent Technologies) was used for probe set summarization and robust multiarray average (RMA) normalization procedures. The differentially expressed genes were selected to have a $>1.5$-fold change difference between the compared groups (average value), a $<10 \%$ FDR using BenjaminiHochberg corrected moderated t-test and $P<.05$.

\section{In silico analysis of genomic evolution}

Genomic conservation was analyzed by downloading Multiz Alignments of 100 Vertebrates from the UCSC Genome Browser (GRCh38/hg38 Assembly, chr22:46,492,389-,46,493,270). Sequence identity was determined using the CLC Genomic Workbench 8.5.4. A phylogenetic tree was generated (Algorithm $=$ UPGMA, Distance measure $=$ Jukes-Cantor, Bootstrap $=100$ Replicates), and identity was calculated by summarizing the distance from Homo sapiens.

\section{Statistical and survival analysis}

Statistical analyses were carried out with GraphPad Prism 7 and SPSS software. To determine whether the data followed a normal Gaussian distribution, the Shapiro-Wilk normality test was performed. P values were determined with a paired/unpaired t-test (normal distribution) or the Wilcoxon matched-pairs signed rank test for paired non-normal distribution data and the MannWhitney-Wilcoxon for un-paired data (non-normal distribution). Linear correlation between gene 
expressions was performed using Pearson correlation coefficient (normal distribution) or nonparametric Spearman correlation (non-normal distribution). All tests were two-sided, and P values $<0.05$ were considered statistically significant.

The Kaplan-Meier method was used to plot and evaluate patients' prognostic outcome. Receiver operating characteristic (ROC) curves with Youden's Index for overall survival/recurrence free survival were established to determine optimal cut-off values for CCAT2, BOP1, PES1 and WDR12. The log-rank test was performed for statistical univariate analysis of prognostic variables. In multivariate analyses, a Cox proportional hazard model was used to identify parameters with a statistically significant influence on survival. 


\section{Supplementary Figure 1}

A

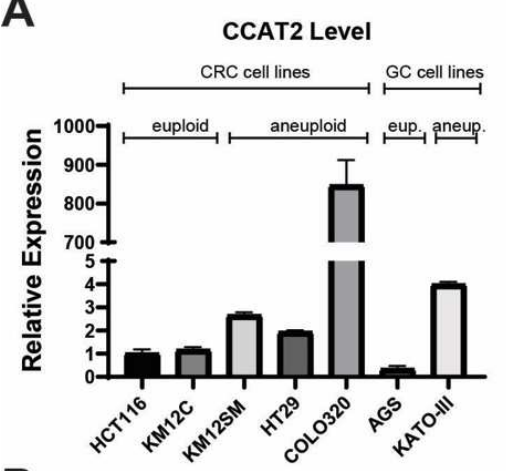

B

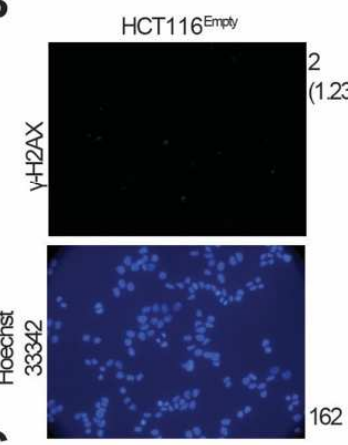

C

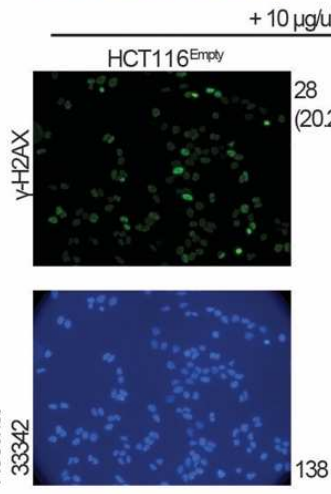

$\mathbf{F}$
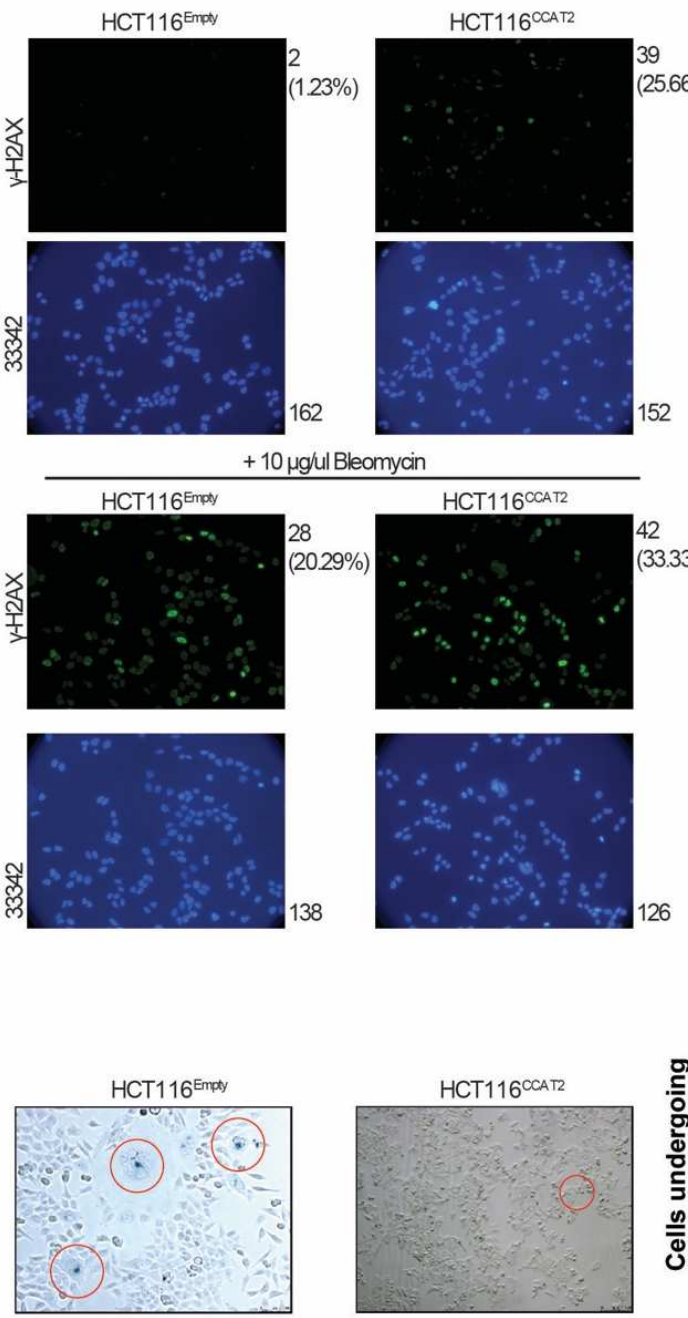

G

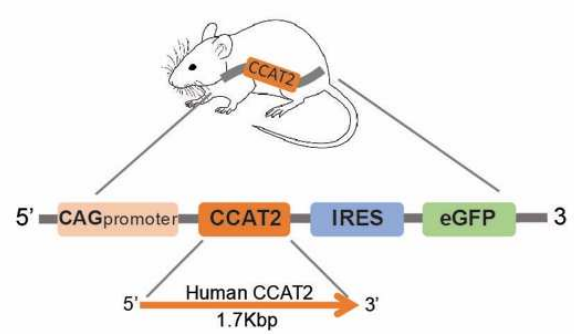

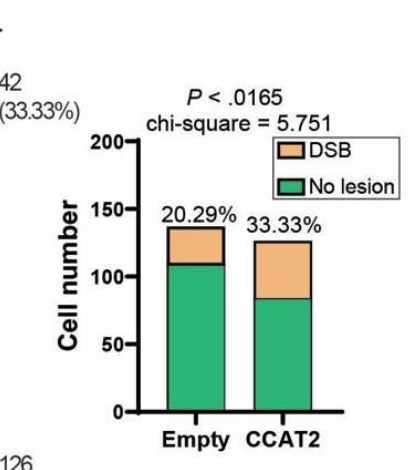

Senescence assay
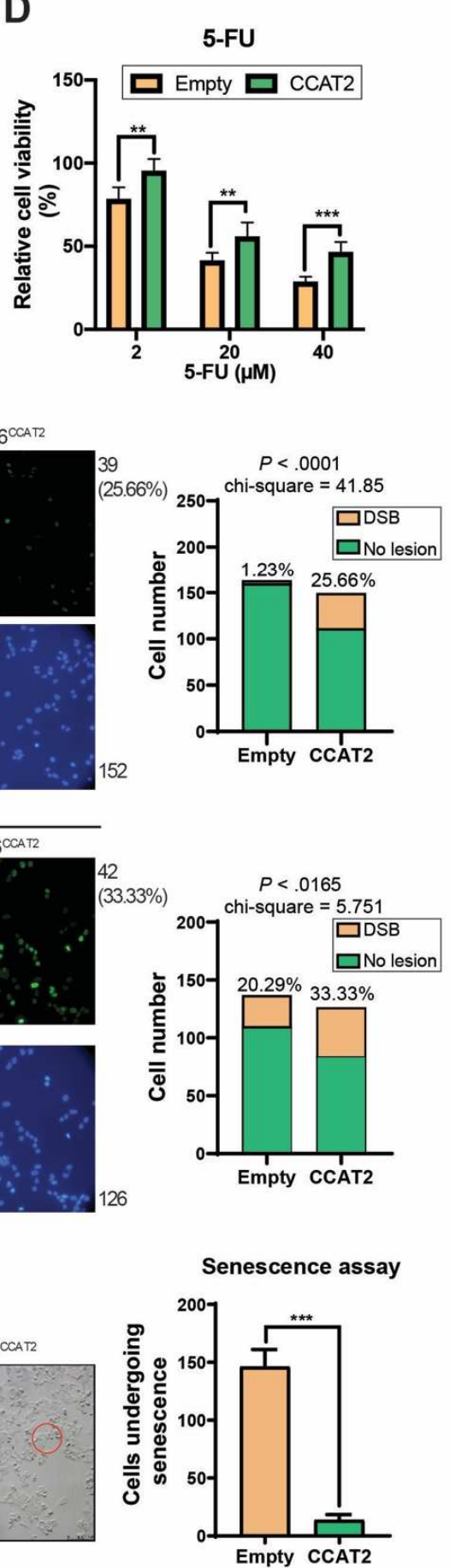

H

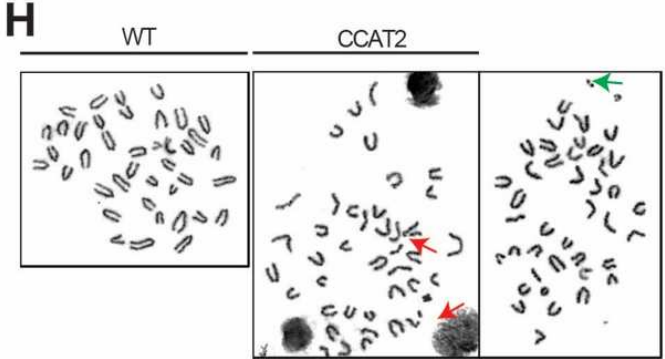

K
E

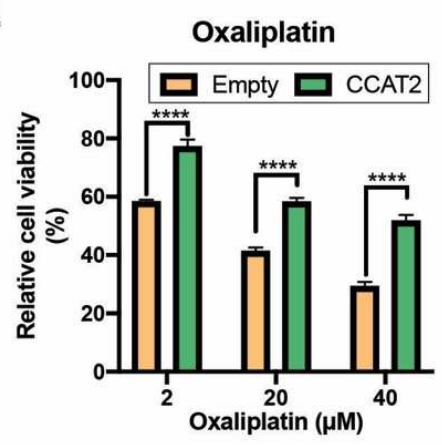

I
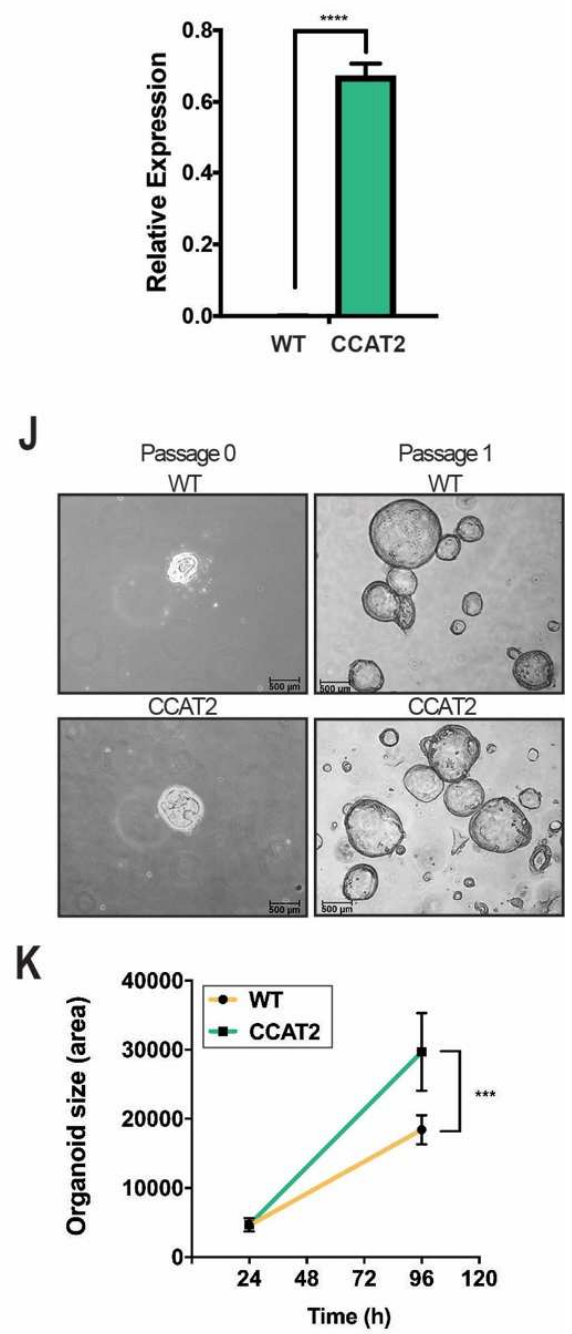
Supplementary Figure 1. Related to Figure 1. A. CCAT2 expression in colorectal cancer (CRC) and gastric cancer (GC) cell lines used in the present study. B. Images of the $\gamma$-H2AX assay for double strand breaks (DSB) without Bleomycin treatment in HCT116 Empty and HCT116 ${ }^{\text {CCAT2 }}$. C. Images of the $\gamma$-H2AX assay for DSB after 2 hours of exposure to $10 \mu \mathrm{g} / \mathrm{ml}$ of Bleomycin in HCT116Empty and HCT116CCAT2. D. Effect of 5-Fluorouracil (5-FU) on cell proliferation of HCT116 ${ }^{\text {Empty }}$ and CCAT2 clones. Cells were treated with three different concentrations of 5-FU $(2,20$ and $40 \mu \mathrm{M})$ and after 48 hours cell viability was determined using the MTT assay. E. Effect of oxaliplatin on cell proliferation of HCT116 Empty and CCAT2 clones. Cells were treated with three different concentrations of oxaliplatin $(2,20$ and $40 \mu \mathrm{M})$ and after 48 hours cell viability was determined using the MTS assay. F. Representative images (in red circles) for senescence assay in HCT116Empty and HCT116 ${ }^{\text {CCAT2 }}$ and the corresponding statistical analysis. G. Schematic representation of CCAT2-plasmid inserted into the mouse genome. H. Representative metaphase from the bone marrow of WT mice and CCAT2 transgenic mouse model that developed myelodysplastic syndrome. Red arrows indicate chromosomal breaks and green arrows chromosomal fragments. I. CCAT2 expression in colon organoids from WT and CCAT2 transgenic mice. J. Representative inverted microscopy images of colon organoids of WT and CCAT2 transgenic mouse model immediately after they were established and after 1 passage. K. Proliferation rate of colon organoids from WT and from CCAT2 transgenic mice. Data are represented as mean values \pm SD. ( $\left.{ }^{* * *} P<.001\right),\left({ }^{* * * *} P<.0001\right)$. Student's t test. 


\section{Supplementary Figure 2}

A

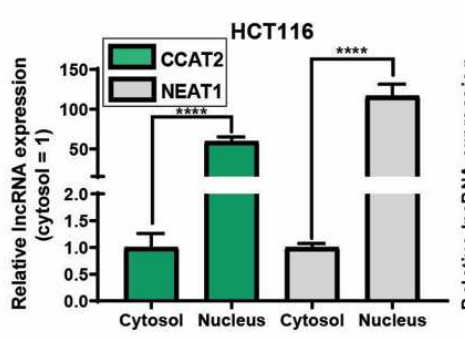

D

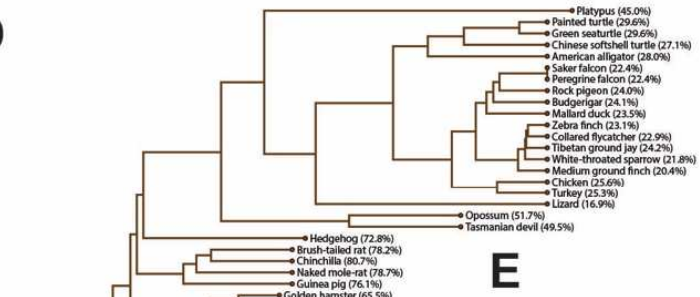

кM12SM

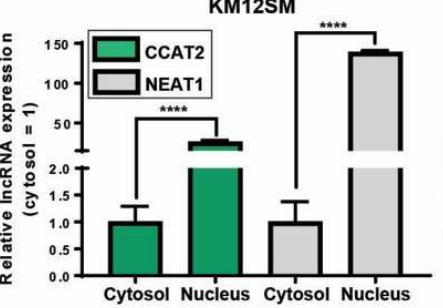

C

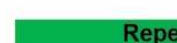

B

COLO320
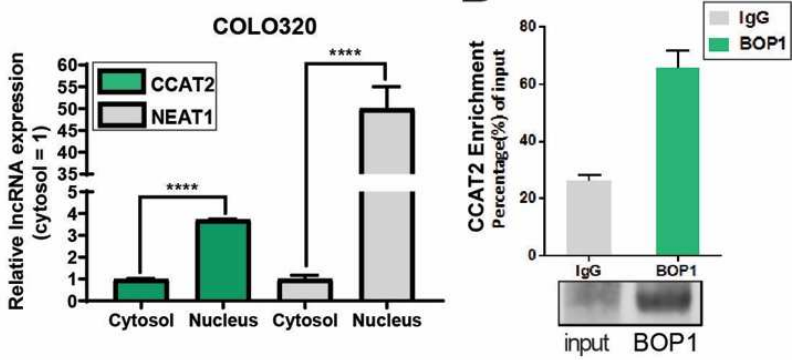

WD 1

Position

$411-450$
$452-492$

$532-576$
$577-615$

618-657

$661-700$
$716-746$

\begin{tabular}{|c|}
\hline Length (aa \\
\hline 40 \\
\hline 41 \\
\hline 45 \\
\hline 39 \\
\hline 40 \\
\hline 40 \\
\hline 31 \\
\hline
\end{tabular}

WD 6

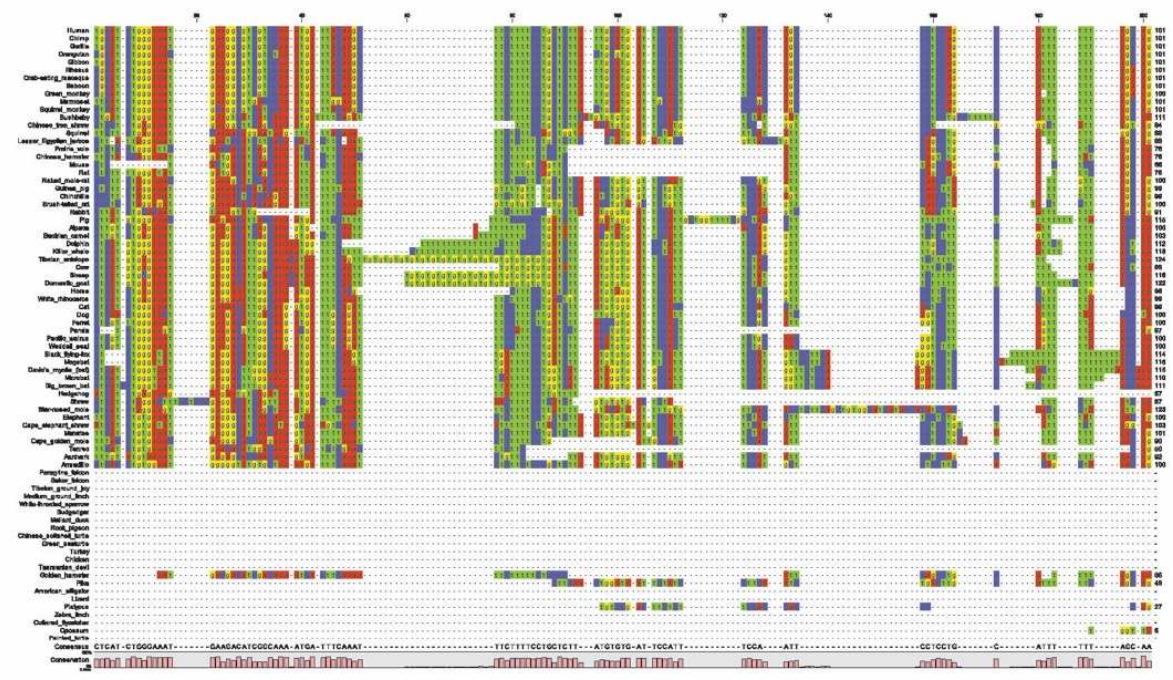

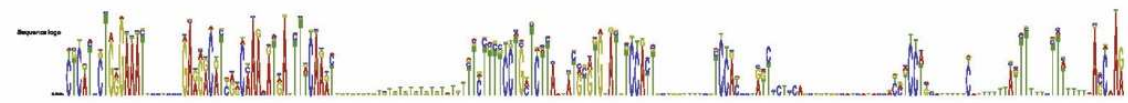
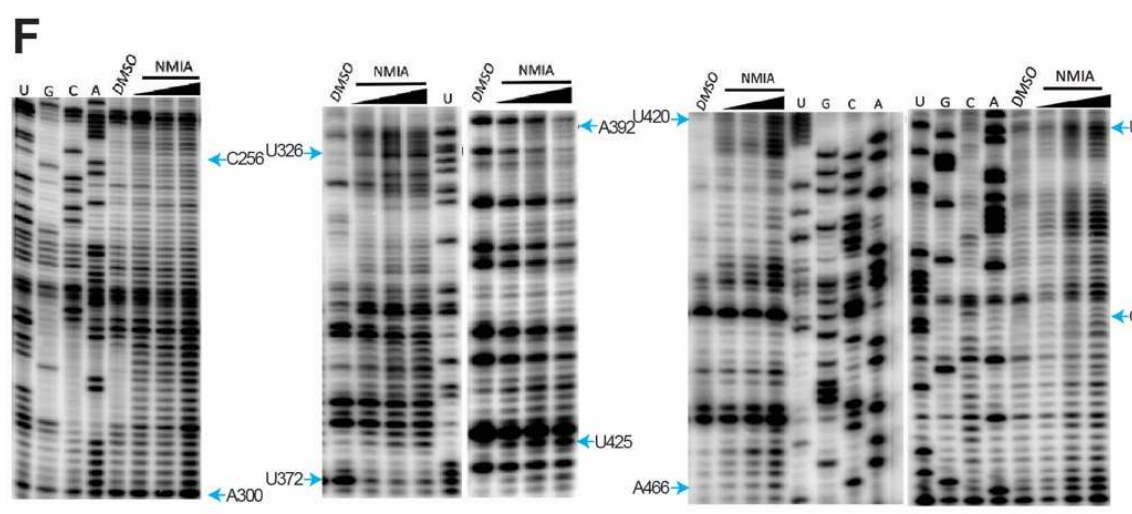

G 
Supplementary Figure 2. Related to Figure 2. A. The nuclear and cytoplasmic localization of CCAT2 and NEAT1 (positive control) in HCT116, KM12SM and COLO320 cells as measured by qRTPCR. B. RIP assays showing that BOP1 interacts with CCAT2 in HCT116 cells. The qRT-PCR results of RIP assays are shown in the upper panel. Western-blot data, to check the immunoprecipitation efficiency, are shown in the lower panel. C. The positions and length of the seven WD repeats of BOP1. D. Evolutionary conservation of full length CCAT2 (Homo sapiens is underlined in red). E. Conservation of the motif 3 of CCAT2. F. Representative gels for the SHAPE probing of CCAT2 330430 fragment with different primers. Lanes from left to right were gels probed with primer 1, 2, 3, 4, 5. U, G, C, A sequencing reactions performed using dideoxy-terminating nucleotides. Control in DMSO; NMIA concentration gradient from $32.5 \mathrm{mmol}$ to $130 \mathrm{mmol}$. G. Quantification of the SHAPE probing data gels for CCAT2 330-430 fragment. Band intensities visualized by gel electrophoresis were quantified using SAFA, version 1.1. $\left({ }^{* * *} P<.0001\right)$. Student's t test. 


\section{Supplementary Figure 3}

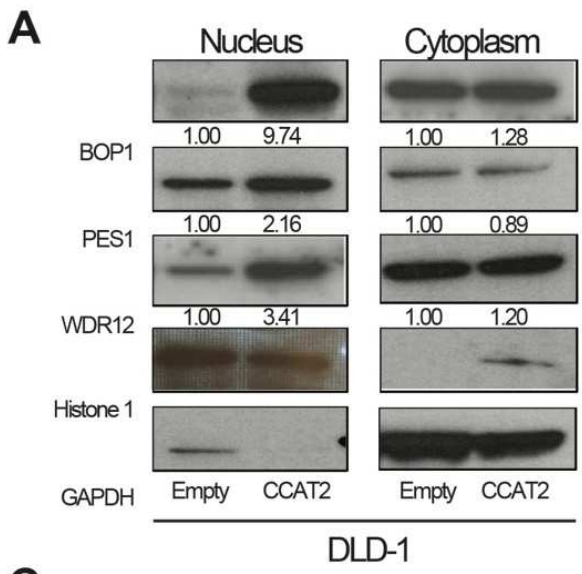

C

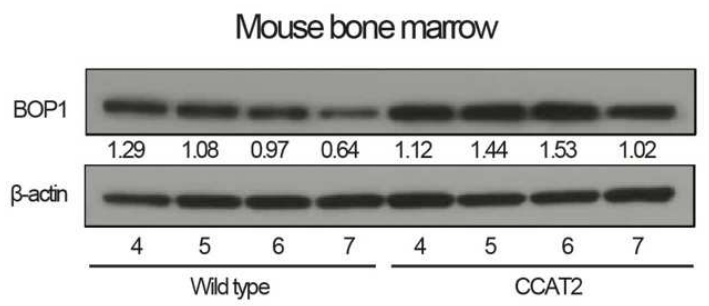

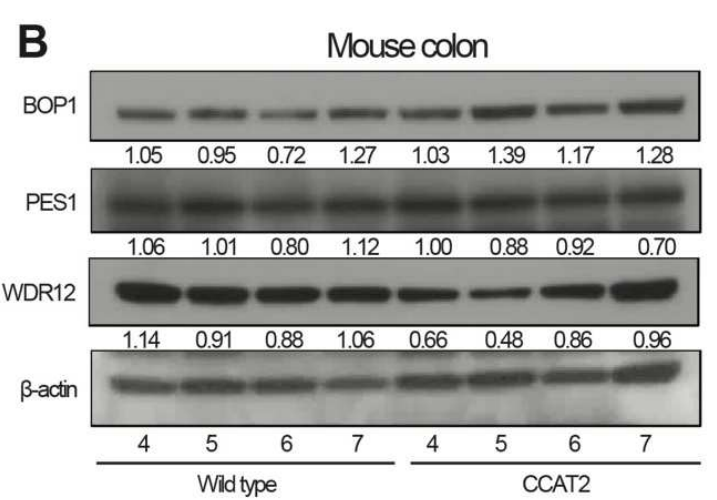

D

E

\begin{tabular}{cccccccc}
\hline Transcript & chromosome & $\begin{array}{c}\text { binding } \\
\text { site(3's) }\end{array}$ & $\begin{array}{c}\text { binding } \\
\text { site(5's) }\end{array}$ & $\begin{array}{c}\text { binding } \\
\text { sites }\end{array}$ & $\begin{array}{c}\text { prediction } \\
\text { score(0-1) }\end{array}$ & $\begin{array}{c}\text { SNP } \\
\text { site }\end{array}$ & $\begin{array}{c}\text { Methylation } \\
\text { site }\end{array}$ \\
\hline ENST00000569669.5 & 8 & 144291213 & 144291220 & ccACGTG & 1 & NO & NO \\
ENST00000569669.5 & 8 & 144292306 & 144292313 & gcACGTG & 1 & NO & NO \\
ENST00000569669.5 & 8 & 144291212 & 144291220 & gcCACGTg & 1 & NO & NO \\
\hline
\end{tabular}

$\mathbf{F}$

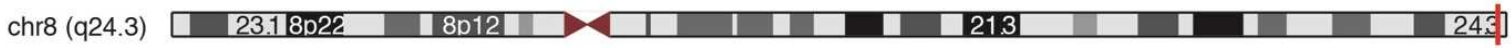

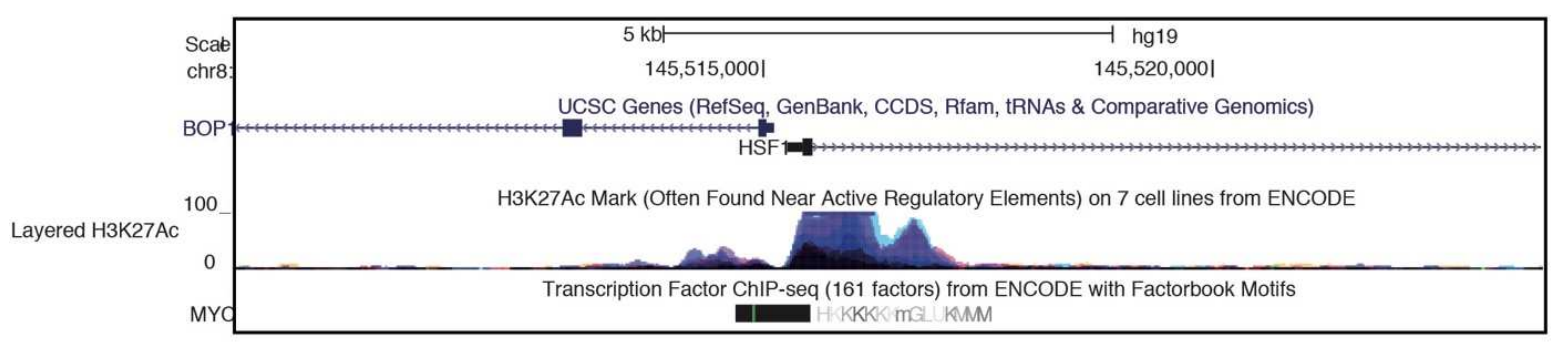

G

MYC tet-on system

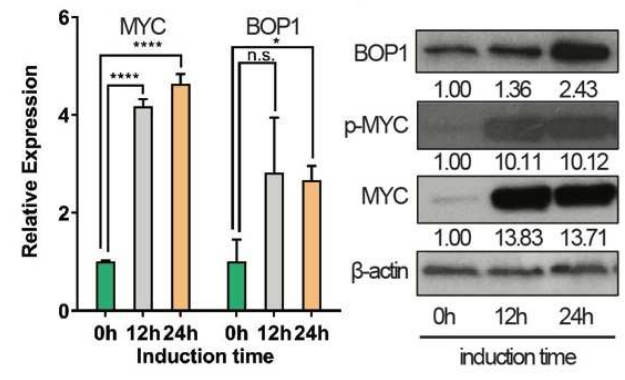


Supplementary Figure 3. Related to Figure 3. A. The nuclear and cytoplasmic localization of PES1, BOP1, and WDR12 (PeBoW) complex was determined by Western-blot of fractionated protein lysate from DLD-1 $1^{\text {Empty }}$ and DLD-1CCAT2 cells. B. The protein expression of PeBoW complex components in the colon of CCAT2 transgenic mice (WT $=4$, CCAT2 $=4)$. C. The protein expression of BOP1 in the bone marrow of CCAT2 transgenic mice (WT = 4, CCAT2 = 4). D. MYC is one of the predicted transcription factors of BOP1 (from GCBI GENERADAR). E. Predicted binding sites of MYC around the genomic location of BOP1. F. CHIP-Seq data (UCSC Genome Browser on Human Feb. 2009 (GRCh37/hg19) Assembly) showing MYC (Cluster Score (out of 1000): 1000, Position: chr8:145514703-145515524, Band: 8q24.3, Genomic Size: 822) binding site in the genomic region around BOP1. G. The expression of BOP1, MYC and p-MYC at 0,12 and 24 hours in HCT116 cells with an inducible c-MYC expression system. Data are represented as mean values \pm SD. (ns, not significant), $\left({ }^{*} P<.05\right),\left({ }^{* * * *} P<.0001\right)$. Student's t test. 


\section{Supplementary Figure 4}

A
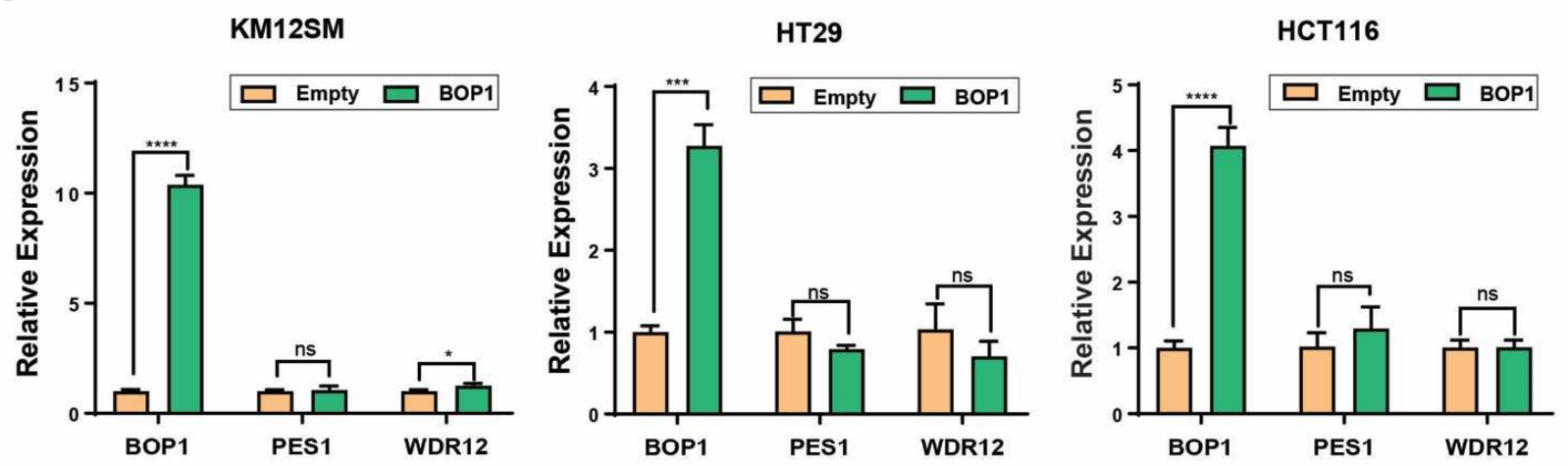

B

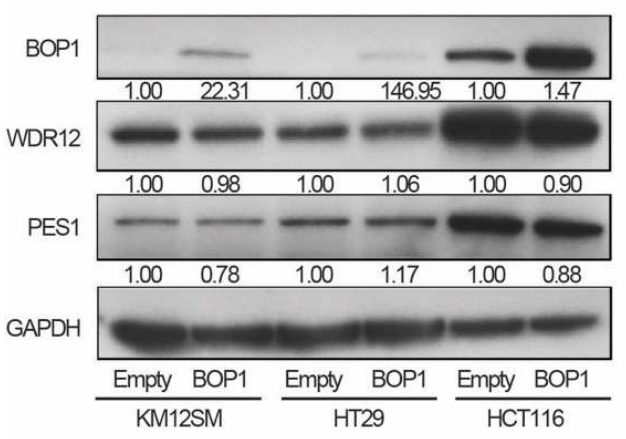

C
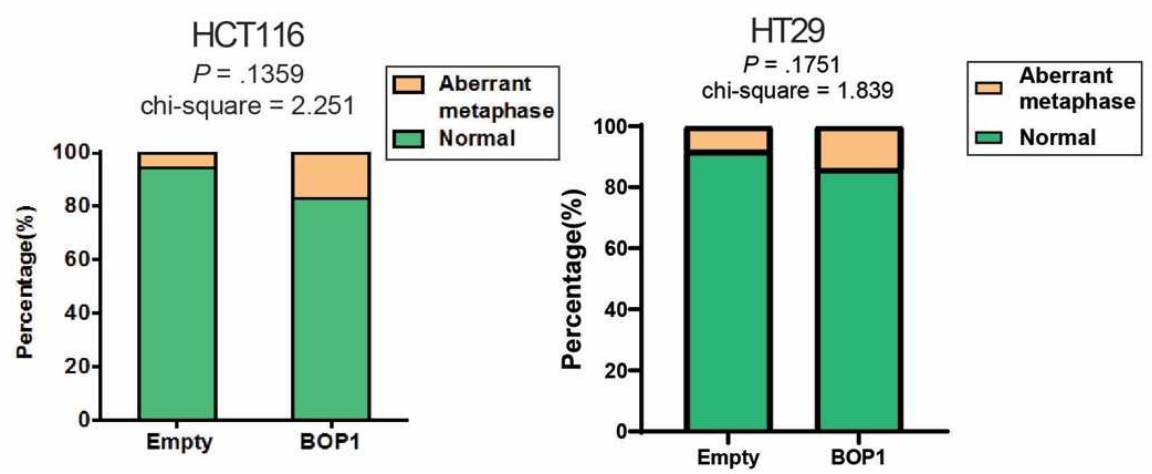
Supplementary Figure 4. Related to Figure 4. A. mRNA expression of PES1, BOP1, and WDR12 (PeBoW) complex members in KM12SM, HT29 and HCT116 Empty and BOP1 clones analyzed by qRT-PCR. B. Protein expression of PeBoW complex members in KM12SM, HT29 and HCT116 Empty and BOP1 clones analyzed by Western blot. C. Cytogenetic analysis showing that the frequency of cells exhibiting chromosome abnormalities (including chromosomal breaks, fusions and polyploidy) in HCT116 and HT29 Empty versus BOP1 clones after a short-term of passaging (5-10) has no significant difference. At least 35 interphase nuclei were analyzed for each clone. Data are represented as mean values \pm SD. (ns, not significant), $\left({ }^{*} P<.05\right),\left({ }^{* * *} P<.001\right),\left({ }^{* * * *} P<\right.$ .0001). Student's t test. 


\section{Supplementary Figure 5}

A

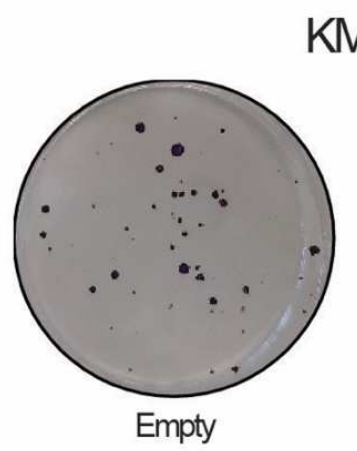

B

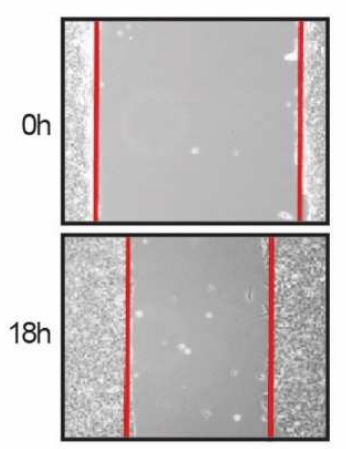

scramble
KM12SM

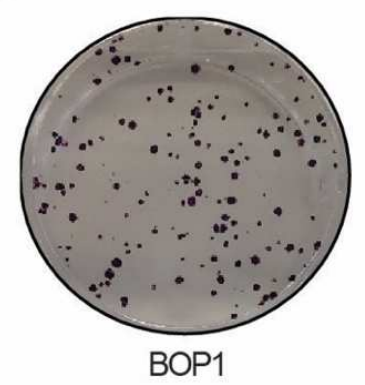

HCT116

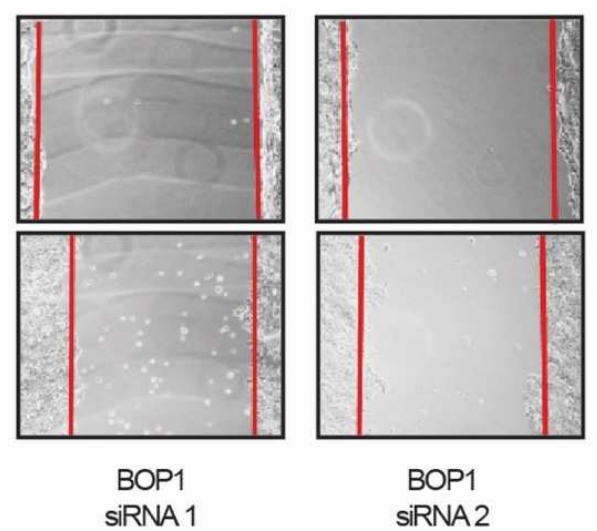

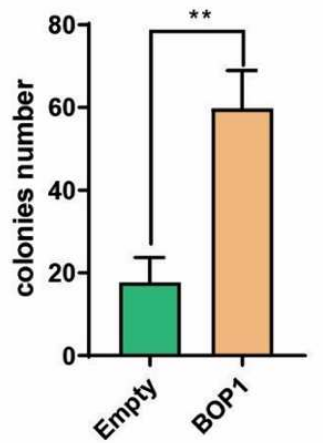

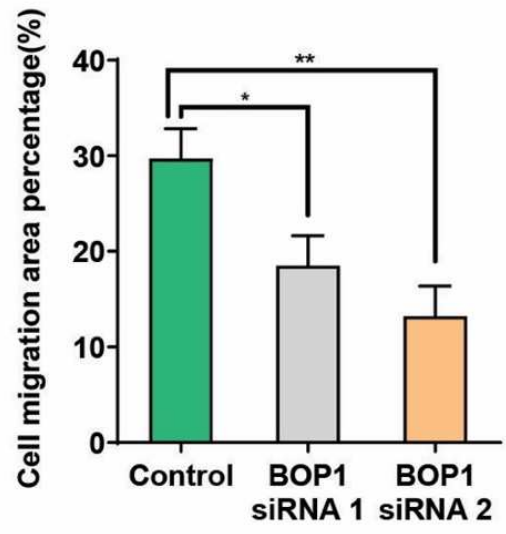


Supplementary Figure 5. Related to Figure 5. A. Up-regulation of $B O P 1$ increases the number of colonies in KM12SM ${ }^{\mathrm{BOP} 1}$ clones. B. Scratch assay showed that transient BOP1 knock-down inhibits the migration capacity of HCT116. Data are represented as mean values \pm SD. $\left({ }^{*} P<.05\right),\left({ }^{* *} P<\right.$ .01). Student's t test. 


\section{Supplementary Figure 6}

A

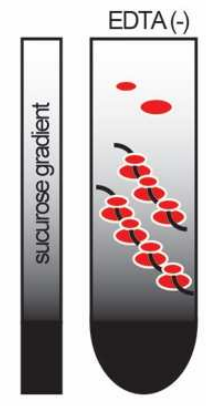

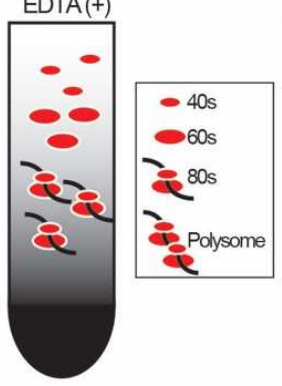

B

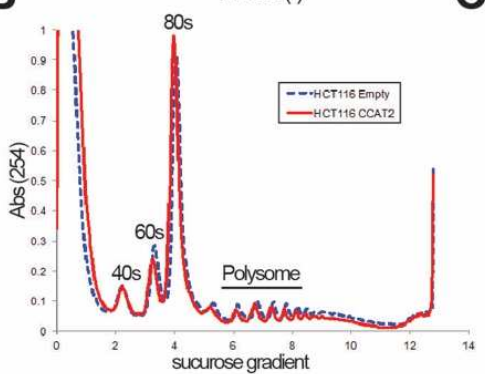

C

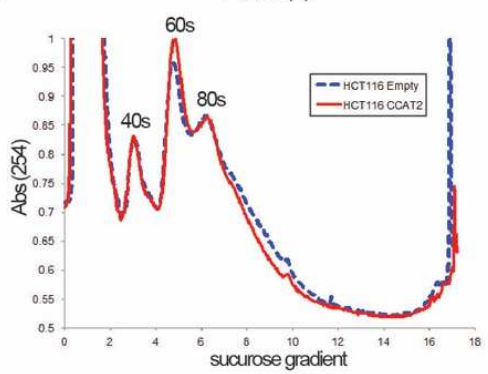

E

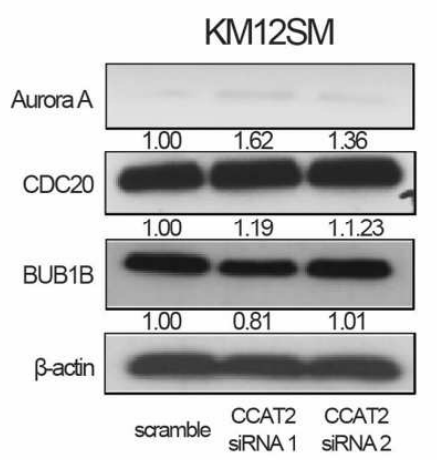

$\mathbf{F}$

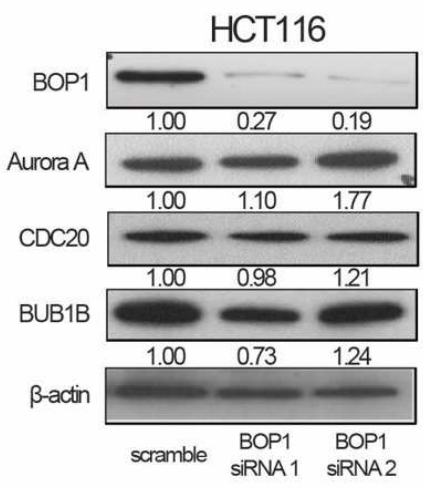

$\mathrm{H}$

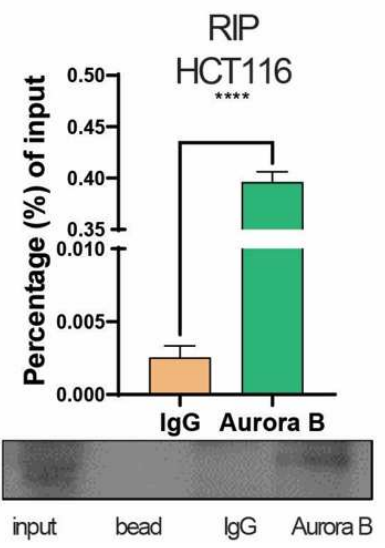

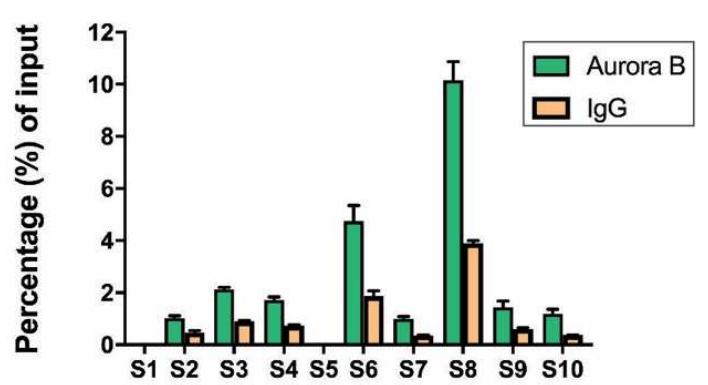


Supplementary Figure 6. Related to Figure 6. A. Schematic representation of Ribosome profiling analysis with or without EDTA. B. Ribosome profiling analysis without EDTA of HCT116Empty and HCT116 $6^{\text {CAT2 }}$ cells. C. Ribosome profiling analysis with EDTA of HCT116 Empty and HCT116CCAT2 cells. D. Correlation matrix between the Aurora family gene expression, PES1, BOP1, and WDR12 (PeBoW) complex components gene expression and index of CIN at whole chromosome level in the CRC cohort from TCGA database. E. The protein levels of key regulators of the chromosomal segregation mechanism (AURKA, CDC20, and BUB1B) were determined by Western blot in KM12SM cells after CCAT2 knock-down. F. The protein levels of key regulators of the chromosomal segregation mechanism (AURKA, CDC20, and BUB1B) were determined by Western blot in HCT116 cells after BOP1 knock-down. G. CHIP-Seq data (UCSC Genome Browser on Human Feb. 2009 (GRCh37/hg19) Assembly) showing MYC (Cluster Score (out of 1000): 595, Position: chr17:8113496-8114099, Band: 17p13.1, Genomic Size: 604) binding site in the 5' region of $A U R K B$. H. RIP assay followed by qRT-PCR was performed to check the interaction of CCAT2 with AURKB. HCT116 cell lysates were precipitated with AURKB antibodies and IgG was used as negative control, and were analyzed by Western blot. I. RNase I digestion followed by RIP assay, quantified by qRT-PCR to detect which CCAT2 segment interacts with AURKB. $(* * * * P<.0001)$. Student's t test. 


\section{Supplementary Figure 7}

A

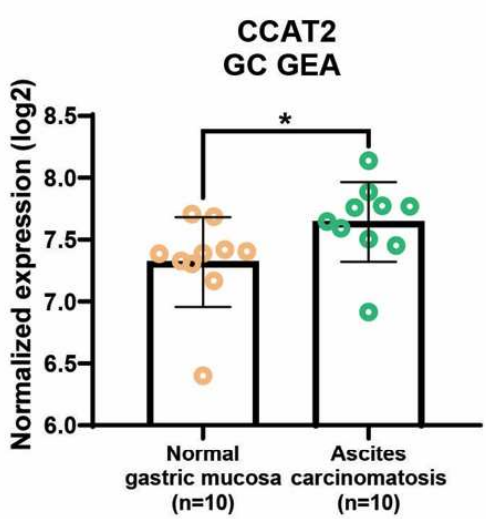

C

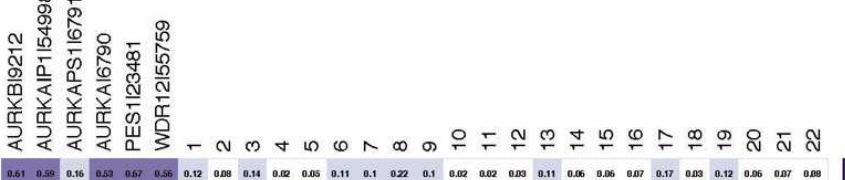
BOP1/23246 AURKBI9212

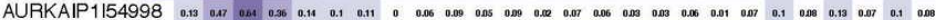

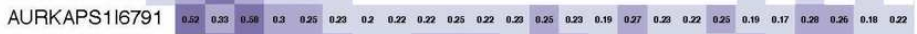
AURKAl6790

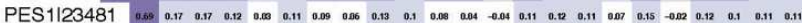

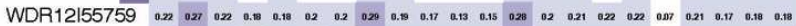
\begin{tabular}{llllllllllllllllll}
1 & 0.52 \\
\hline
\end{tabular}

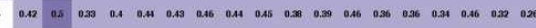

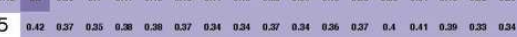

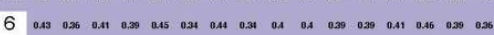

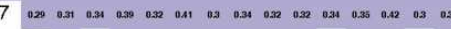

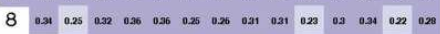

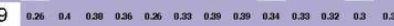

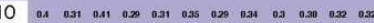

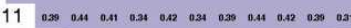

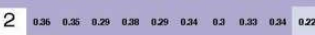

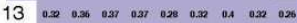

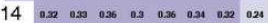

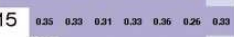

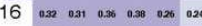

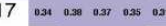
18 o.37 o. 0.31 0.4 19 o.st of 020 $20 \quad 0.0000$ $\left[\begin{array}{c}1 \\ 0.75 \\ 0.5\end{array}\right.$

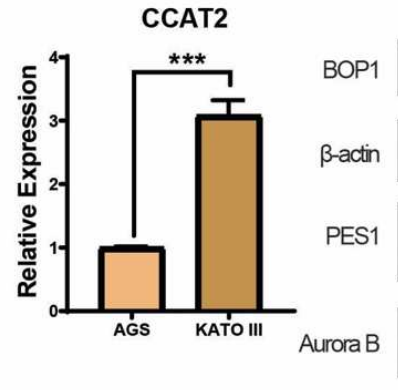
AGS KATO-III

D
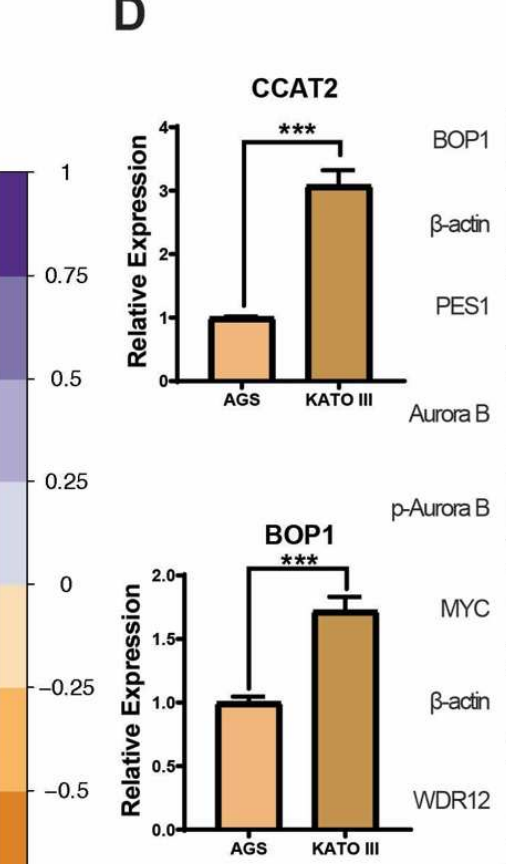

CCAT2 GC PDX/PDO primary metastatic

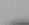
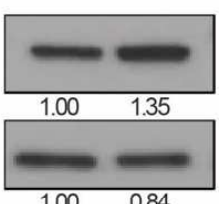
$1.00 \quad 0.84$
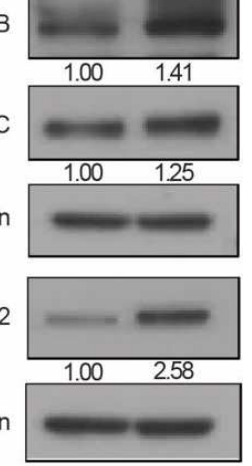
Supplementary Figure 7. Related to Figure 6. A. Log2 normalized expression of CCAT2 obtained from GEA comparing normal gastric mucosa with malignant ascites from GC patients. B. CCAT2 expression in cells derived from GC patients and paired PDX/PDO C. Correlation matrix between the Aurora family gene expression, PES1, BOP1, and WDR12 (PeBoW) complex components gene expression and index of CIN at whole chromosome level in the GC cohort from TCGA database. D. The expression of CCAT2, PeBoW complex members, AURKB, pAURKB and MYC analyzed by qRTPCR and Western blot in gastric cancer cell lines. AGS is a gastric cancer cell line derived from primary tumor and KATO III is derived from metastatic lymph nodes. Data are represented as mean values $\pm \mathrm{SD} .\left({ }^{*} P<.05\right),\left({ }^{* * *} P<.001\right),\left({ }^{* * *} P<.0001\right)$. Student's test. 


\section{Supplementary Figure 8} A

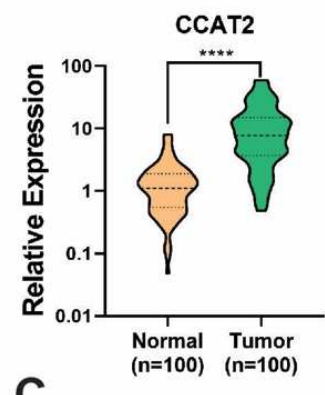

C

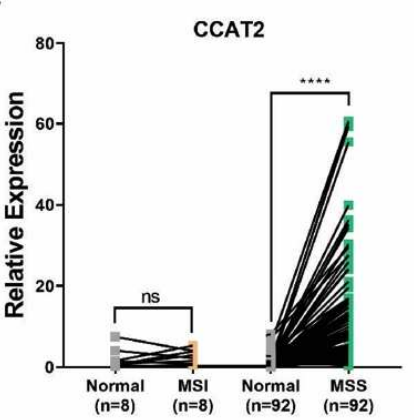

E
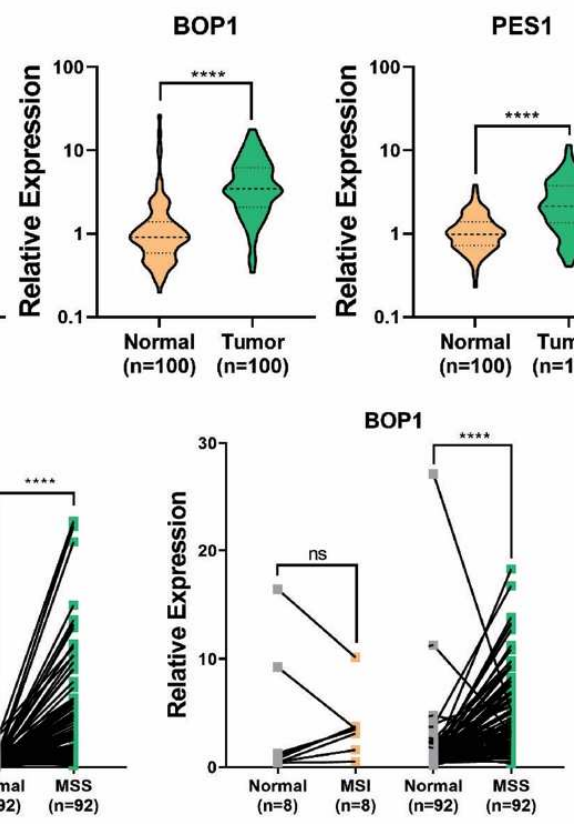

$\mathbf{F}$
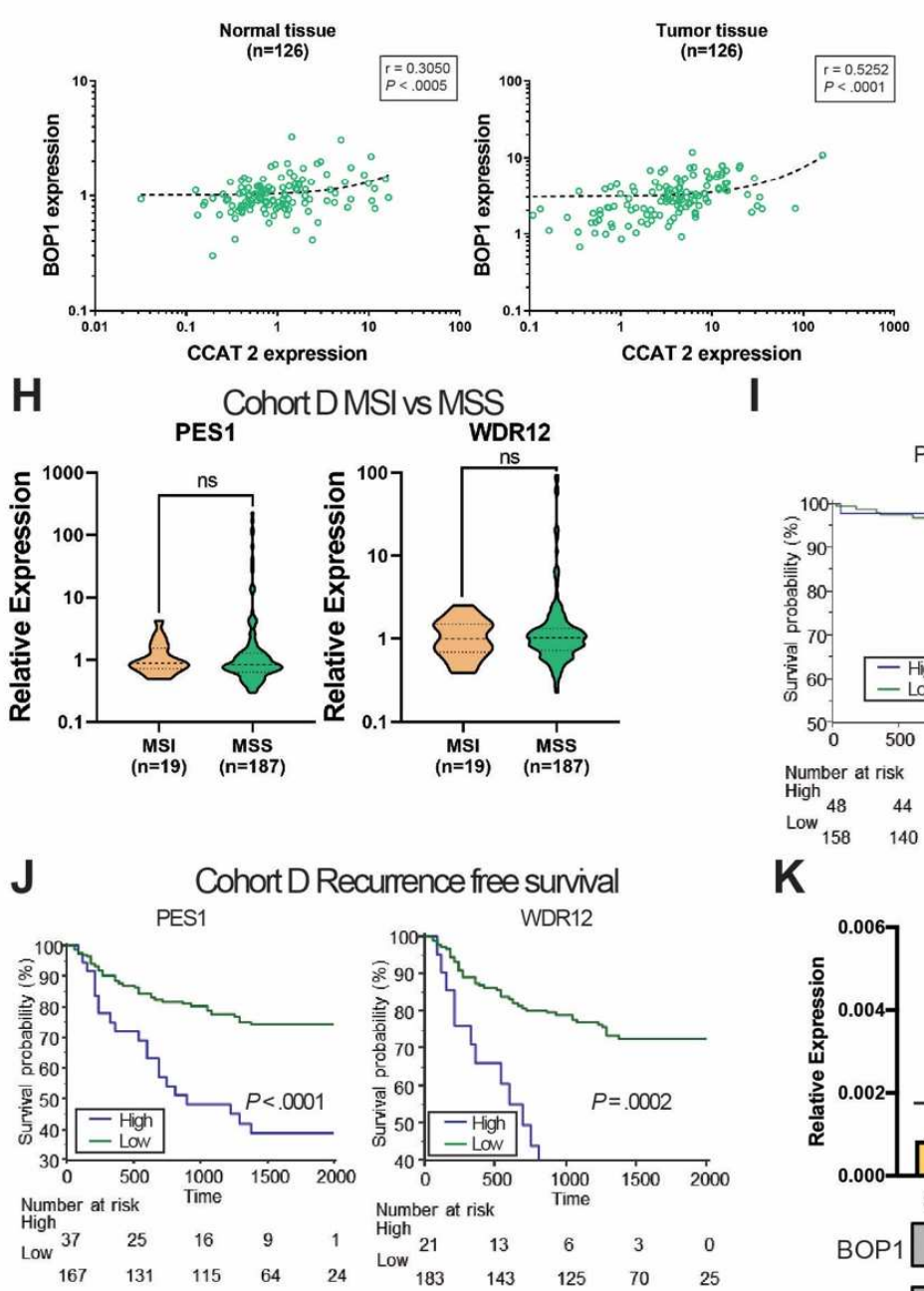

CohortB Normal vs Tumor

I

K
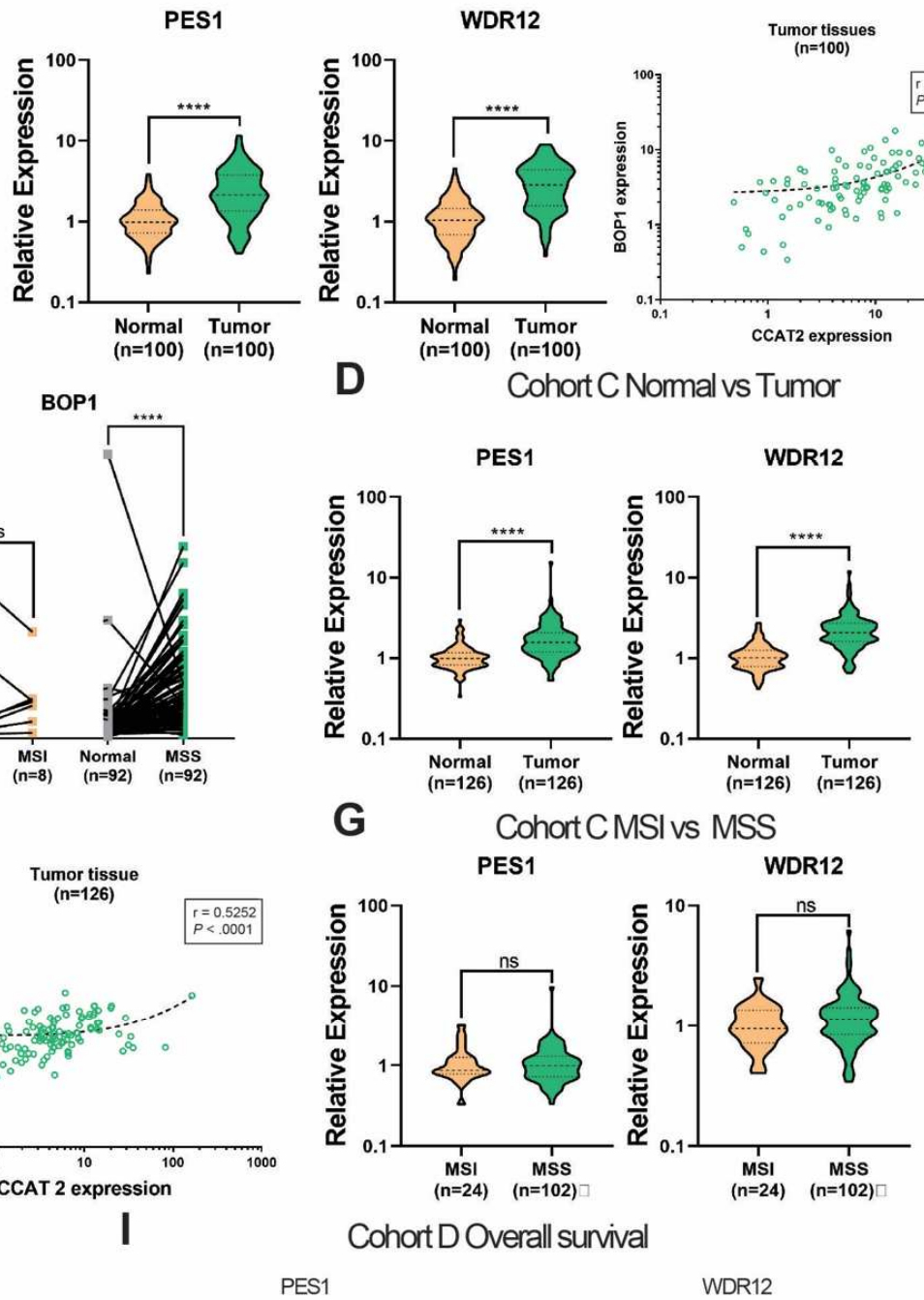

G CohortCMSIvs MSS PES1

WDR12

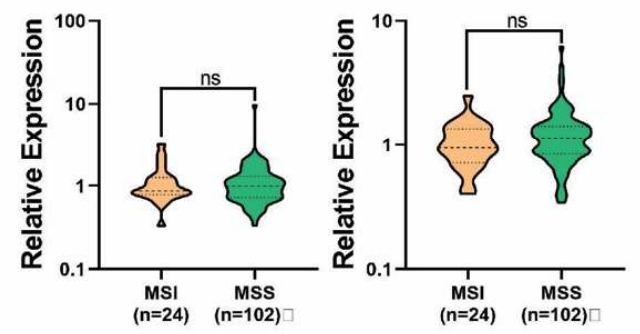

CohortD Overall survival PES1
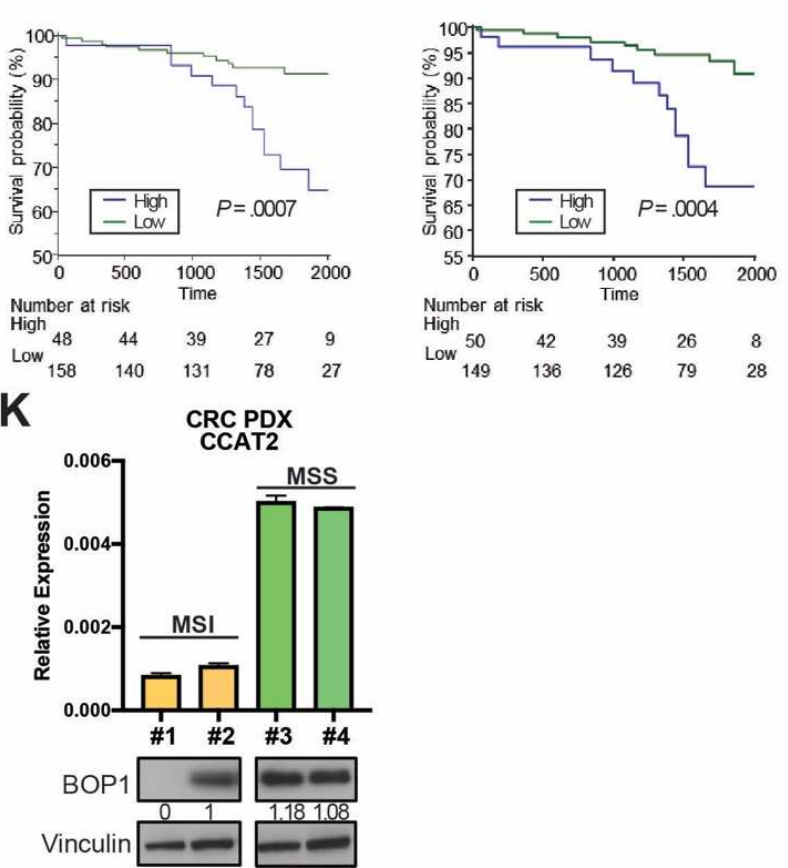
Supplementary Figure 8. Related to Figure 7. A. RNA expression of CCAT2, BOP1, PES1 and WDR12 in 100 paired primary CRC tumor tissues and the adjacent non-tumor colon/rectum was analyzed by qRT-PCR (Cohort B). The expression of the RNA has been normalized to $\beta$-actin. Wilcoxon test for non-normal distributed paired samples was performed. B Spearman correlation between the expression of the IncRNA CCAT2 and the mRNA BOP1 in tumor tissues in Cohort B. C. BOP1 and CCAT2 expression in paired normal and MSI (MSI-H) or normal and MSS (MSS/MSI-L) CRC tissues. Wilcoxon test for non-normal distributed paired samples was performed. D. PES1 and WDR12 expression in adjacent normal and tumor tissues from Cohort C. Wilcoxon test for nonnormal distributed paired samples was performed. E. Spearman correlation between the expression of the IncRNA CCAT2 and the mRNA BOP1 in normal tissues in Cohort C. F. Spearman correlation between the expression of the lncRNA CCAT2 and the mRNA BOP1 in tumor tissue in Cohort C. G. PES1 and WDR12 expression in MSI (MSI-H) and MSS (MSS/MSI-L) tumors of Cohort C. Mann-Whitney test for non-normal distributed un-paired samples was performed. H. PES1 and WDR12 expression in MSI (MSI-H) and MSS (MSS/MSI-L) tumors of Cohort D. Mann-Whitney test for non-normal distributed un-paired samples was performed. I. Kaplan-Meier overall survival curves and J. recurrence free survival curves of CRC patients from the Cohort D, expressing high (blue) or low (green) levels of PES1 and WDR12. Time is expressed in months. K. The expression of the IncRNA CCAT2 and the protein expression of BOP1 in two MSI PDX (\#1 and \#2) and two MSS PDX (\#3 and \#4). Data are represented as violin plots. (ns, not significant), $\left({ }^{* * * *} P<.0001\right)$. Student's t test and Mann-Whitney-Wilcoxon test. 
Supplementary Table 1. Clinical and pathological characteristics of the Cohort A (TCGA CRC cohort).

\begin{tabular}{lcc}
\hline Variable & & $\mathrm{n}$ \\
\hline Gender & male & 280 \\
Age & female & 257 \\
Tumor location & median (range) & $66(31-90)$ \\
& right side colon & 184 \\
& left side colon & 192 \\
UICC Stage & rectum & 49 \\
& I & 92 \\
& II & 201 \\
MSI/MSS status & III & 154 \\
& IV & 73 \\
& MSS & 150 \\
& MSI-L & 39 \\
& MSI-H & 29 \\
\hline
\end{tabular}


Supplementary Table 2. Clinical and pathological characteristics of Cohort B.

\begin{tabular}{lcc}
\hline Variable & & $\mathrm{n}$ \\
\hline Gender & male & 66 \\
Age & female & 34 \\
Tumor location & median (range) & $64(33-85)$ \\
& right side colon & 22 \\
& left side colon & 29 \\
Differentiation grade & rectum & 49 \\
& well & 56 \\
& moderate & 36 \\
T stage & poor & 3 \\
& others & 5 \\
& T1 & 17 \\
Lymph node metastasis & T2 & 12 \\
& T3 & 63 \\
Distant metastasis & T4 & 8 \\
& pogative & 48 \\
UICC Stage & M0 & 52 \\
& M1 & 91 \\
Lymphatic invasion & I-II & 4 \\
Venous invasion & III & 46 \\
MSI/MSS status & IV & 9 \\
& negative & 65 \\
& positive & 35 \\
& negative & 65 \\
& positive & 35 \\
& MSS & 82 \\
& MSI-L & 10 \\
& MSI-H & 8 \\
\hline & & \\
& & 46 \\
& &
\end{tabular}


Supplementary Table 3. Clinical and pathological characteristics of Cohort C.

\begin{tabular}{lcc}
\hline Variable & & $\mathrm{n}$ \\
\hline Gender & male & 76 \\
Age & female & 50 \\
Tumor location & median (range) & $64(30-84)$ \\
Differentiation grade & proximal & 47 \\
& distal & 79 \\
& well (G1) & 32 \\
T stage & moderate (G2) & 68 \\
& poor (G3) & 19 \\
& other (missing, G4, multiple grades) & 7 \\
Lymph node metastasis & T1 & 1 \\
Distant metastasis & T2 & 22 \\
& T3 & 95 \\
UICC Stage & T4 & 8 \\
& negative & 69 \\
& positive & 57 \\
MSI/MSS status & M0 & 91 \\
& M1 & 35 \\
& I & 17 \\
& II & 42 \\
& III & 32 \\
& IV & 35 \\
& MSI & 24 \\
& MSS & 102 \\
\hline
\end{tabular}


Supplementary Table 4. Clinical and pathological characteristics of Cohort D.

\begin{tabular}{lcc}
\hline Variable & & $\mathrm{n}$ \\
\hline Gender & male & 120 \\
Age at operation & female & 86 \\
Tumor location & median (range) & $70(33-93)$ \\
& right side colon & 47 \\
& left side colon & 79 \\
Tumor size (mm) & rectum & 80 \\
Histological type & median (range) & $45(10-140)$ \\
& intestinal & 184 \\
T stage & diffuse & 22 \\
& T2 & 9 \\
Lymph node metastasis & T3 & 141 \\
& T4 & 56 \\
UICC Stage & negative & 111 \\
& positive & 95 \\
Lymphatic invasion & II & 111 \\
& III & 95 \\
Venous invasion & negative & 92 \\
& positive & 114 \\
MSI/MSS status & negative & 21 \\
& positive & 185 \\
& MSI & 19 \\
& MSS & 187 \\
\hline
\end{tabular}


Supplementary Table 5. Primers and siRNAs used in this study.

\begin{tabular}{|c|c|c|}
\hline Gene & Sequence & Description \\
\hline CCAT2 F1 (human) & 5' GGGCACTAGACTGGGAATTAG 3' & PCR Primer \\
\hline CCAT2 R1 (human) & 5' AGGGAGCTGAGATAGGAAGAG 3' & PCR Primer \\
\hline BOP1 F2 (human) & 5' GCCACAAGATGCACGTACCT 3' & PCR Primer \\
\hline BOP1 R2 (human) & 5' TTCCTGGATGAAGCGTCCGTA 3' & PCR Primer \\
\hline PES1 F (human) & 5' GGGCATTTATCCCCATGAACC 3' & PCR Primer \\
\hline PES1 R (human) & 5' CACCTTGTATTCACGGAACTTGT 3' & PCR Primer \\
\hline WDR12 F (human) & 5' CAGAGGAATGGATCTTGACTGGT 3' & PCR Primer \\
\hline WDR12 R (human) & 5' CAGTGTAGGGCTTTCACTTTGT 3' & PCR Primer \\
\hline B0P1-del6-264 F (human) & 5' GGCGGGTTCGCGGATGGGCTGGATCC 3' & PCR Primer \\
\hline BOP1-del6-264 R (human) & 5' GGATCCAGCCCATCCGCGAACCCGCC 3' & PCR Primer \\
\hline BOP1-delWD1 F (human) & 5' CCTGGTCTACAGGGTTCCCGTGGGGG 3' & PCR Primer \\
\hline BOP1-delWD1 R (human) & 5' CCCCCACGGGAACCCTGTAGACCAGG 3' & PCR Primer \\
\hline BOP1-delWD2 F (human) & 5' CGCTGTGTGAGGACTGTTGGCAGCACAGATCAG 3' & PCR Primer \\
\hline BOP1-delWD2 R (human) & 5' CTGATCTGTGCTGCCAACAGTCCTCACACAGCG 3' & PCR Primer \\
\hline BOP1-delWD3 F (human) & 5' CGGCTGCGCATCCGCAGCCACGGA 3' & PCR Primer \\
\hline BOP1-delWD3 R (human) & 5' TCCGTGGCTGCGGATGCGCAGCCG 3' & PCR Primer \\
\hline BOP1-delWD4 F (human) & 5' CAGAGTCCGTTCCGCCTGATGCCCAACTGC 3' & PCR Primer \\
\hline BOP1-delWD4 R (human) & 5' GCAGTTGGGCATCAGGCGGAACGGACTCTG 3' & PCR Primer \\
\hline BOP1-delWD5 F (human) & 5' CTCACCAAGAAGCTGATGATGCTGAGACACCACAAG 3' & PCR Primer \\
\hline BOP1-delWD5 R (human) & 5' CTTGTGGTGTCTCAGCATCATCAGCTTCTTGGTGAG 3' & PCR Primer \\
\hline BOP1-delWD6 F (human) & 5’ ATACAGGATGCTGAGAAACCCCTTGCTGGTGC 3' & PCR Primer \\
\hline BOP1-delWD6 R (human) & 5' GCACCAGCAAGGGGTTTCTCAGCATCCTGTAT 3' & PCR Primer \\
\hline BOP1-del265-427 F (human) & 5’ GGTGCACGCCATCAAGCTGGTTTCAGGCTCTG 3' & PCR Primer \\
\hline BOP1-del265-427 R (human) & 5' CAGAGCCTGAAACCAGCTTGATGGCGTGCACC 3' & PCR Primer \\
\hline CCAT2-EcoRI F (human) & 5' CCGgaattcccgaggtgatcaggtggact 3' & PCR Primer \\
\hline CCAT2-SalI R (human) & 5' CGCgtcgacgtcttctgggctgatgttgc 3' & PCR Primer \\
\hline CCAT2q-1 F (human) & 5' TAATACGACTCACTATAGACCCAGCAAGTTTCTCAGGA 3' & PCR Primer \\
\hline CCAT2q-1 R (human) & 5' CATTTTTCAGCAATCAGGTCAA 3' & PCR Primer \\
\hline CCAT2q-2 F (human) & 5' TAATACGACTCACTATAGAGACACCAAGAGGGAGGTATCA 3' & PCR Primer \\
\hline CCAT2q-2 R (human) & 5' TGGCTCTTGACTTCCAGTCC 3' & PCR Primer \\
\hline CCAT2q-3 F (human) & 5' TAATACGACTCACTATAGAACTTTCCCAGCCTCGTTCT & PCR Primer \\
\hline CCAT2q-3 R (human) & 5' GGCTGTGGAAGTGGAATCAT & PCR Primer \\
\hline CCAT2q-4 F (human) & 5’ TAATACGACTCACTATAGCTCCATAGAGCCTGCAGAGG & PCR Primer \\
\hline CCAT2q-4 R (human) & 5’ ATTGGTCAGAGGTGGAGCTG 3' & PCR Primer \\
\hline
\end{tabular}




\begin{tabular}{|c|c|c|}
\hline CCAT2q-5 F (human) & 5’ TAATACGACTCACTATAGCAGCAGATGAAAGGCACTGA 3' & PCR Primer \\
\hline CCAT2q-5 R (human) & 5' СТСССТСССССАСАТАAАAТ 3' & PCR Primer \\
\hline CCAT2q-6 F (human) & 5' TAATACGACTCACTATAGACCCAGCAAGTTTCTCAGGA 3' & PCR Primer \\
\hline CCAT2q-6 R (human) & 5’ CACAGTTATTGCCTGGAGCA 3' & PCR Primer \\
\hline CCAT2q-7 F (human) & 5’ TAATACGACTCACTATAGGGCATGCCCTACGTAAGTTC 3’ & PCR Primer \\
\hline CCAT2q-7 R (human) & 5' TTTGGGGGTAGGTCAGGAAT 3' & PCR Primer \\
\hline CCAT2q-8 F (human) & 5’ TAATACGACTCACTATAGTGCATTGGTGAGCTGTGTTT 3’ & PCR Primer \\
\hline CCAT2q-8 R (human) & 5' ATGGTGCTGCTGGTAGCTTT 3' & PCR Primer \\
\hline CCAT2q-9 F (human) & 5’ TAATACGACTCACTATAGGCCATAATCATCCCTGAGGA 3' & PCR Primer \\
\hline CCAT2q-9 R (human) & 5' CACCCCAGAGAGATGACACC 3' & PCR Primer \\
\hline CCAT2q-10 F (human) & 5’ TAATACGACTCACTATAGTTGTTGGGGTTTGATCCTTT 3' & PCR Primer \\
\hline CCAT2q-10 R (human) & 5' CAAGCACTTGGAGCACACAT 3' & PCR Primer \\
\hline Aurora B F (human) & 5’ ACAGACGGCTCCATCTGGCCT 3' & PCR Primer \\
\hline Aurora B R (human) & 5’ GGCAGCTGTGGGCTGGACATT 3' & PCR Primer \\
\hline c-MYC F (humane) & 5' GGCTCCTGGCAAAAGGTCA 3' & PCR Primer \\
\hline c-MYC R (humane) & 5’ CTGCGTAGTTGTGCTGATGT 3' & PCR Primer \\
\hline$\beta$-actin F (human) & 5’ CATGTACGTTGCTATCCAGGC 3’ & PCR Primer \\
\hline$\beta$-actin R (human) & 5' CTCCTTAATGTCACGCACGAT 3’ & PCR Primer \\
\hline U6 F (human) & 5’ CTCGCTTCGGCAGCACA 3' & PCR Primer \\
\hline U6 R (human) & 5’ AACGCTTCACGAATTTGCGT 3’ & PCR Primer \\
\hline GAPDH F (human) & 5’ CTGGGCTACACTGAGCACC 3' & PCR Primer \\
\hline GAPDH R (human) & 5' AAGTGGTCGTTGAGGGCAATG 3' & PCR Primer \\
\hline BOP1 F (mouse) & 5' CAGCTCTGATGAGGAGGACATTCGGAAC 3' & PCR Primer \\
\hline B0P1 R (mouse) & 5’ CAACCTGCTCATCAGTTAGCCG 3' & PCR Primer \\
\hline mACTB F (mouse) & 5’ CCTGTGCTGCTCACCGAGGC 3’ & PCR Primer \\
\hline mACTB R (mouse) & 5’ GACCCCGTCTCTCCGGAGTCCATC 3’ & PCR Primer \\
\hline HPRT1 F (mouse) & 5’ ACATTGTGGCCCTCTGTGTG 3’ & PCR Primer \\
\hline HPRT1 R (mouse) & 5’ TTATGTCCCCCGTTGACTGA 3' & PCR Primer \\
\hline SiRNA & Target Sequence/Producer ID & \\
\hline CCAT2 siRNA 1 & 5’ AGGTGTAGCCAGAGTTAAT 3' & siRNA target sequence \\
\hline CCAT2 SIRNA 2 & 5’ AGGAAGAGGTTAAGCAATT 3' & siRNA target sequence \\
\hline BOP1 siRNA 1 & s198254 & Producer ID \\
\hline BOP1 siRNA 2 & s198255 & Producer ID \\
\hline
\end{tabular}


Supplementary Table 6. Antibodies used in this study.

\begin{tabular}{|c|c|}
\hline Antibodies & Product information \\
\hline BOP1 (rabbit) & Abcam (ab86982) \\
\hline BOP1 (mouse) & Santa Cruz (SC-390672) \\
\hline BOP1 (IP) & Abcam (ab86652) \\
\hline WDR12 & Abcam (ab95070) \\
\hline PES1 & Abcam(ab88543) \\
\hline PES1 (IP) & Santa Cruz (SC-166300) \\
\hline MYC & Millipore (06-340) \\
\hline pSer62 cMyc & Cell Signaling Technologies 13748 \\
\hline Aurora Kinase B (rabbit) & Cell Signaling Technologies 3094 \\
\hline Aurora Kinase B (mouse) & Santa Cruz (SC-65987) \\
\hline P Thr232 Aurora Kinase B & Invitrogen PA5-38557 \\
\hline$\gamma$-tubulin & Cell Signaling Technologies 3873T \\
\hline$\beta$-actin & Cell Signaling Technologies 3700T \\
\hline GAPDH & Santa Cruz (SC51905) \\
\hline Histone H1 & Santa Cruz (SC34464) \\
\hline
\end{tabular}


Supplementary Table 7. Cell lines used in this study (colon cancer and gastric cancer cell lines).

\begin{tabular}{|l|c|c|c|c|c|}
\hline Cell line & CCAT2 expression* & CIN & $\begin{array}{c}\text { Modal } \\
\text { chromosome no. }\end{array}$ & TP53 & References \\
\hline HCT116 & Low & - & $\mathbf{4 5 / 4 6}$ & WT & 25,26 \\
\hline KM12C & Low & - & $\mathbf{4 5}$ & H179R & $1,27,28$ \\
\hline KM12SM & High & $\mathbf{+}$ & $\mathbf{7 5 - 8 2}$ & H179R & $1,27,28$ \\
\hline COLO320 & Very high & $\mathbf{+}$ & $\mathbf{5 1}$ & $\mathbf{R 2 4 8 W}$ & 29 \\
\hline HT29 & High & $\mathbf{+}$ & $\mathbf{7 0 ~ ( 3 n )}$ & R273H & 25,30 \\
\hline AGS & Low & - & $\mathbf{4 9}$ & WT & $31-33$ \\
\hline KATO-III & High & $\mathbf{+}$ & tetraploid range & Genomic deletion & $31,34,35$ \\
\hline
\end{tabular}

CIN: chromosomal instability. ${ }^{*}$ see Supplementary Figure 1A for data. 
Supplementary Table 8. List of proteins identified by mass spectrometry to specifically bind to CCAT2 after MS2 pull-down assay*

\begin{tabular}{|c|c|c|c|}
\hline Accession & Gene & Subcellular location & \# interaction hits by Mass Spec \\
\hline B4DX30 & ACSL5 & Nucleus, Mitochondria & 2 \\
\hline E9PRN9 & BOP1 & Nucleus, Nucleoli & 1 \\
\hline B4DDM6 & BUB3 & Nucleoplasm & 1 \\
\hline H3BTZ5 & CNN2 & Nucleoplasm, Actin filaments, Cytosol & 1 \\
\hline B4DLW8 & DDX5 & Nucleoplasm & 4 \\
\hline Q13347 & EIF3I & Nucleoplasm, Cytosol & 2 \\
\hline H7BY36 & EWSR1 & Nucleus, Nucleoli & 2 \\
\hline Q8IW50-7 & FAM219A & $\begin{array}{l}\text { Nucleoplasm, Golgi apparatus, } \\
\text { Vesicles }\end{array}$ & 1 \\
\hline B4DY13 & GTPBP4 & Nucleoli & 2 \\
\hline 094992 & HEXIM1 & Nucleoplasm, Vesicles & 1 \\
\hline 060814 & HIST1H2BK & Nucleoplasm, Cytosol & 10 \\
\hline Q5T7C4 & HMGB1 & Nucleus & 1 \\
\hline H7C1J8 & HNRNPA3 & Nucleoplasm & 1 \\
\hline P25205 & MCM3 & Nucleoplasm & 6 \\
\hline Q15233-2 & NONO & Nucleoplasm & 2 \\
\hline H0Y653 & NOP56 & Nucleoli fibrillar center & 1 \\
\hline G3V1R4 & NSUN2 & Nucleus & 3 \\
\hline K7ELW0 & PARK7 & Nucleus, Cytosol & 2 \\
\hline E7ETC2 & PPP3CA & Nucleoplasm, Cytosol & 2 \\
\hline H0YMZ1 & PSMA4 & Nucleus, Vesicles, Cytosol & 2 \\
\hline P28074 & PSMB5 & Nucleus, Centrosome & 2 \\
\hline Q06124-2 & PTPN11 & Nucleus, Nucleoli, Actin filaments, Cytosol & 1 \\
\hline K7EIJ4 & RANBP3 & Nucleoplasm & 1 \\
\hline B4DWG0 & RCBTB2 & Nucleus, Golgi apparatus & 1 \\
\hline I3L4R8 & RPA1 & Nucleoplasm & 1 \\
\hline P40429 & RPL13A & Nucleoli, Cytosol & 1 \\
\hline F8VUA6 & RPL18 & Nucleoli, Endoplasmic reticulum, Cytosol & 2 \\
\hline E9PKZ0 & RPL8 & $\begin{array}{l}\text { Nucleoli, Endoplasmic reticulum, } \\
\text { Cytosol }\end{array}$ & 2 \\
\hline G3XAA9 & RPS6KA4 & Nucleus, Cytosol & 1 \\
\hline K7EPT6 & TAF15 & Nucleoplasm & 2 \\
\hline G3V448 & TMX1 & Nucleoli, Endoplasmic reticulum & 1 \\
\hline Q13263 & TRIM28 & Nucleoplasm & 10 \\
\hline J3KT19 & USP10 & Nucleoplasm, Cytosol & 1 \\
\hline B1AHC7 & XRCC6 & Nucleoplasm & 7 \\
\hline P27348 & YWHAQ & Nucleoplasm, Cytosol & 4 \\
\hline C9JN71 & ZNF878 & Nucleus, Nucleoli & 3 \\
\hline
\end{tabular}

* - MS2 Empty Pull down in all the instances gave 0 hits. 
Supplementary Table 9. CINdex analysis at the chromosome level of the CRC TCGA cohort and correlation between the genes of the Aurora family, PeBoW complex and CIN at chromosomal level.

\begin{tabular}{|c|c|c|c|c|c|c|}
\hline $\begin{array}{l}\text { Chr. } \\
\text { number }\end{array}$ & Gene & $\begin{array}{l}\text { Correlation Test - T } \\
\text { statistic }\end{array}$ & P-value & $\begin{array}{l}\text { Correlation } \\
\text { coefficient }\end{array}$ & $\begin{array}{l}\text { Confidence } \\
\text { interval } 1\end{array}$ & $\begin{array}{l}\text { Confidence } \\
\text { interval } 2\end{array}$ \\
\hline 20 & AURKA & 9.7 & $3.20 \mathrm{E}-19$ & 0.501 & 0.41 & 0.58 \\
\hline 20 & WDR12 & 4.6 & $6.50 \mathrm{E}-06$ & 0.266 & 0.15 & 0.37 \\
\hline 13 & AURKA & 4.0 & 7.97E-05 & 0.234 & 0.12 & 0.34 \\
\hline 20 & AURKAPS1 & 4.0 & $8.80 \mathrm{E}-05$ & 0.232 & 0.12 & 0.34 \\
\hline 2 & WDR12 & 3.6 & 0.0003271 & 0.213 & 0.10 & 0.32 \\
\hline 20 & BOP1 & 3.5 & 0.0005195 & 0.206 & 0.09 & 0.32 \\
\hline 20 & AURKC & -3.4 & 0.0007570 & -0.200 & -0.31 & -0.08 \\
\hline 7 & AURKA & 3.3 & 0.0009951 & 0.196 & 0.08 & 0.31 \\
\hline 8 & BOP1 & 3.1 & 0.0019504 & 0.184 & 0.07 & 0.30 \\
\hline 7 & AURKC & -3.1 & 0.0019925 & -0.184 & -0.29 & -0.07 \\
\hline 19 & AURKAIP1 & -2.9 & 0.0045704 & -0.169 & -0.28 & -0.05 \\
\hline 2 & AURKA & 2.8 & 0.0052080 & 0.167 & 0.05 & 0.28 \\
\hline 17 & AURKA & 2.8 & 0.0060698 & 0.164 & 0.05 & 0.28 \\
\hline 13 & AURKC & -2.7 & 0.0067611 & -0.162 & -0.27 & -0.05 \\
\hline 20 & AURKAIP1 & -2.7 & 0.0069726 & -0.161 & -0.27 & -0.04 \\
\hline 10 & WDR12 & 2.7 & 0.0070027 & 0.161 & 0.04 & 0.27 \\
\hline 6 & AURKAPS1 & 2.7 & 0.0078359 & 0.159 & 0.04 & 0.27 \\
\hline 15 & AURKAIP1 & -2.7 & 0.0081522 & -0.158 & -0.27 & -0.04 \\
\hline 13 & WDR12 & 2.5 & 0.0127088 & 0.149 & 0.03 & 0.26 \\
\hline 8 & AURKA & 2.5 & 0.0129917 & 0.148 & 0.03 & 0.26 \\
\hline 12 & AURKA & 2.5 & 0.0133298 & 0.148 & 0.03 & 0.26 \\
\hline 1 & AURKAIP1 & -2.5 & 0.0137918 & -0.147 & -0.26 & -0.03 \\
\hline 5 & AURKAPS1 & 2.5 & 0.0138759 & 0.147 & 0.03 & 0.26 \\
\hline 8 & AURKAIP1 & -2.4 & 0.0160611 & -0.144 & -0.26 & -0.03 \\
\hline 5 & WDR12 & 2.4 & 0.0164290 & 0.143 & 0.03 & 0.26 \\
\hline 12 & AURKC & -2.4 & 0.0174374 & -0.142 & -0.25 & -0.03 \\
\hline 17 & AURKAPS1 & 2.4 & 0.0187027 & 0.140 & 0.02 & 0.25 \\
\hline 22 & AURKAPS1 & 2.3 & 0.0202989 & 0.139 & 0.02 & 0.25 \\
\hline 12 & WDR12 & 2.3 & 0.0223718 & 0.136 & 0.02 & 0.25 \\
\hline 15 & AURKC & -2.3 & 0.0241227 & -0.135 & -0.25 & -0.02 \\
\hline 8 & AURKAPS1 & 2.3 & 0.0244076 & 0.134 & 0.02 & 0.25 \\
\hline 6 & AURKA & 2.2 & 0.0252607 & 0.134 & 0.02 & 0.25 \\
\hline 19 & PES1 & -2.2 & 0.0258461 & -0.133 & -0.25 & -0.02 \\
\hline 15 & AURKB & -2.2 & 0.0261202 & -0.133 & -0.25 & -0.02 \\
\hline 4 & AURKA & 2.2 & 0.0261782 & 0.133 & 0.02 & 0.25 \\
\hline 1 & AURKAPS1 & 2.2 & 0.0277008 & 0.132 & 0.01 & 0.25 \\
\hline 18 & WDR12 & 2.2 & 0.0278869 & 0.131 & 0.01 & 0.24 \\
\hline 21 & WDR12 & 2.2 & 0.0310365 & 0.129 & 0.01 & 0.24 \\
\hline 22 & AURKA & 2.1 & 0.0337156 & 0.127 & 0.01 & 0.24 \\
\hline 4 & WDR12 & 2.1 & 0.0355162 & 0.126 & 0.01 & 0.24 \\
\hline 14 & WDR12 & 2.1 & 0.0378901 & 0.124 & 0.01 & 0.24 \\
\hline 8 & AURKC & -2.1 & 0.0379421 & -0.124 & -0.24 & -0.01 \\
\hline 16 & AURKAPS1 & 2.0 & 0.0417412 & 0.122 & 0.00 & 0.24 \\
\hline 4 & AURKAPS1 & 2.0 & 0.0429808 & 0.121 & 0.00 & 0.23 \\
\hline
\end{tabular}

AURKA, aurora kinase A; AURKAIP1, aurora kinase A interacting protein 1; AURKAPS1, aurora kinase A pseudogene 1; AURKB, aurora kinase B, BOP1, BOP1 ribosomal biogenesis factor; PES1 pescadillo ribosomal biogenesis factor 1; WDR12, WD repeat domain 12 . 
Supplementary Table 10. CINdex analysis at the chromosome level of the GC TCGA cohort and correlation between the genes of the Aurora family, PeBoW complex and CIN at chromosomal level.

\begin{tabular}{|c|c|c|c|c|c|c|}
\hline Chr. number & Gene & $\begin{array}{l}\text { Correlation Test - } \\
\text { T statistic }\end{array}$ & P value & $\begin{array}{l}\text { Correlation } \\
\text { coefficient }\end{array}$ & $\begin{array}{l}\text { Confidence } \\
\text { Interval 1 }\end{array}$ & $\begin{array}{l}\text { Confidence } \\
\text { Interval } 2\end{array}$ \\
\hline 20 & AURKA & 7.39 & $8.05 \mathrm{E}-13$ & 0.34 & 0.25 & 0.43 \\
\hline 8 & AURKA & 7.14 & $4.33 \mathrm{E}-12$ & 0.33 & 0.24 & 0.42 \\
\hline 19 & AURKA & 7.01 & $9.73 \mathrm{E}-12$ & 0.33 & 0.24 & 0.41 \\
\hline 2 & AURKA & 6.98 & $1.18 \mathrm{E}-11$ & 0.33 & 0.24 & 0.41 \\
\hline 1 & AURKAPS1 & 6.41 & $3.91 \mathrm{E}-10$ & 0.30 & 0.21 & 0.39 \\
\hline 3 & AURKA & 6.34 & $6.23 \mathrm{E}-10$ & 0.30 & 0.21 & 0.38 \\
\hline 8 & WDR12 & 6.09 & $2.64 \mathrm{E}-09$ & 0.29 & 0.20 & 0.37 \\
\hline 13 & AURKA & 6.08 & $2.83 \mathrm{E}-09$ & 0.29 & 0.20 & 0.37 \\
\hline 1 & AURKA & 5.95 & 5.70E-09 & 0.28 & 0.19 & 0.37 \\
\hline 6 & AURKA & 5.91 & $7.11 \mathrm{E}-09$ & 0.28 & 0.19 & 0.37 \\
\hline 19 & AURKAPS1 & 5.88 & 8.39E-09 & 0.28 & 0.19 & 0.37 \\
\hline 13 & WDR12 & 5.81 & $1.24 \mathrm{E}-08$ & 0.28 & 0.18 & 0.36 \\
\hline 17 & AURKA & 5.71 & $2.20 \mathrm{E}-08$ & 0.27 & 0.18 & 0.36 \\
\hline 13 & AURKAPS1 & 5.68 & $2.54 \mathrm{E}-08$ & 0.27 & 0.18 & 0.36 \\
\hline 10 & AURKA & 5.67 & $2.74 \mathrm{E}-08$ & 0.27 & 0.18 & 0.36 \\
\hline 2 & WDR12 & 5.65 & $3.01 \mathrm{E}-08$ & 0.27 & 0.18 & 0.36 \\
\hline 2 & AURKB & 5.58 & $4.27 \mathrm{E}-08$ & 0.27 & 0.17 & 0.35 \\
\hline 11 & AURKA & 5.58 & $4.31 \mathrm{E}-08$ & 0.27 & 0.17 & 0.35 \\
\hline 20 & AURKAPS1 & 5.45 & $8.49 \mathrm{E}-08$ & 0.26 & 0.17 & 0.35 \\
\hline 16 & AURKAPS1 & 5.32 & $1.74 \mathrm{E}-07$ & 0.25 & 0.16 & 0.34 \\
\hline 7 & AURKA & 5.27 & $2.24 \mathrm{E}-07$ & 0.25 & 0.16 & 0.34 \\
\hline 10 & AURKAPS1 & 5.26 & $2.33 \mathrm{E}-07$ & 0.25 & 0.16 & 0.34 \\
\hline 2 & AURKAPS1 & 5.25 & 2.39E-07 & 0.25 & 0.16 & 0.34 \\
\hline 7 & AURKAPS1 & 5.22 & $2.88 \mathrm{E}-07$ & 0.25 & 0.16 & 0.34 \\
\hline 8 & AURKB & 5.20 & $3.21 \mathrm{E}-07$ & 0.25 & 0.16 & 0.34 \\
\hline 9 & AURKA & 5.14 & $4.32 \mathrm{E}-07$ & 0.25 & 0.15 & 0.33 \\
\hline 22 & AURKA & 5.10 & $5.16 \mathrm{E}-07$ & 0.24 & 0.15 & 0.33 \\
\hline 3 & AURKB & 5.08 & 5.83E-07 & 0.24 & 0.15 & 0.33 \\
\hline 19 & AURKB & 5.00 & $8.52 \mathrm{E}-07$ & 0.24 & 0.15 & 0.33 \\
\hline 5 & AURKA & 4.99 & 8.99E-07 & 0.24 & 0.15 & 0.33 \\
\hline 14 & AURKAPS1 & 4.89 & $1.45 \mathrm{E}-06$ & 0.23 & 0.14 & 0.32 \\
\hline 9 & AURKAPS1 & 4.85 & $1.74 \mathrm{E}-06$ & 0.23 & 0.14 & 0.32 \\
\hline 3 & AURKAPS1 & 4.83 & $1.97 \mathrm{E}-06$ & 0.23 & 0.14 & 0.32 \\
\hline 11 & AURKAPS1 & 4.76 & $2.69 \mathrm{E}-06$ & 0.23 & 0.14 & 0.32 \\
\hline 3 & WDR12 & 4.67 & $4.05 \mathrm{E}-06$ & 0.22 & 0.13 & 0.31 \\
\hline 5 & AURKAPS1 & 4.61 & $5.49 \mathrm{E}-06$ & 0.22 & 0.13 & 0.31 \\
\hline 1 & WDR12 & 4.60 & $5.67 \mathrm{E}-06$ & 0.22 & 0.13 & 0.31 \\
\hline 16 & AURKA & 4.60 & $5.71 \mathrm{E}-06$ & 0.22 & 0.13 & 0.31 \\
\hline 6 & AURKB & 4.59 & $6.00 \mathrm{E}-06$ & 0.22 & 0.13 & 0.31 \\
\hline 15 & AURKAPS1 & 4.58 & $6.22 \mathrm{E}-06$ & 0.22 & 0.13 & 0.31 \\
\hline 8 & AURKAPS1 & 4.55 & $7.06 \mathrm{E}-06$ & 0.22 & 0.13 & 0.31 \\
\hline 14 & AURKA & 4.55 & $7.08 \mathrm{E}-06$ & 0.22 & 0.13 & 0.31 \\
\hline 8 & BOP1 & 4.52 & 8.02E-06 & 0.22 & 0.12 & 0.31 \\
\hline 16 & WDR12 & 4.50 & $8.93 \mathrm{E}-06$ & 0.22 & 0.12 & 0.31 \\
\hline 22 & AURKAPS1 & 4.50 & $8.99 \mathrm{E}-06$ & 0.22 & 0.12 & 0.31 \\
\hline 6 & AURKAPS1 & 4.48 & $9.86 \mathrm{E}-06$ & 0.22 & 0.12 & 0.31 \\
\hline 17 & WDR12 & 4.47 & $1.00 \mathrm{E}-05$ & 0.22 & 0.12 & 0.31 \\
\hline 21 & AURKA & 4.44 & $1.18 \mathrm{E}-05$ & 0.21 & 0.12 & 0.30 \\
\hline 18 & AURKA & 4.34 & $1.81 \mathrm{E}-05$ & 0.21 & 0.12 & 0.30 \\
\hline 15 & WDR12 & 4.28 & $2.33 \mathrm{E}-05$ & 0.21 & 0.11 & 0.30 \\
\hline 17 & AURKB & 4.27 & $2.40 \mathrm{E}-05$ & 0.21 & 0.11 & 0.30 \\
\hline 19 & WDR12 & 4.25 & $2.64 \mathrm{E}-05$ & 0.21 & 0.11 & 0.30 \\
\hline 4 & AURKAPS1 & 4.19 & $3.42 \mathrm{E}-05$ & 0.20 & 0.11 & 0.29 \\
\hline 6 & WDR12 & 4.13 & $4.45 \mathrm{E}-05$ & 0.20 & 0.11 & 0.29 \\
\hline 14 & WDR12 & 4.12 & $4.58 \mathrm{E}-05$ & 0.20 & 0.10 & 0.29 \\
\hline 7 & WDR12 & 4.06 & 0.0001 & 0.20 & 0.10 & 0.29 \\
\hline
\end{tabular}




\begin{tabular}{|c|c|c|c|c|c|c|}
\hline 13 & AURKB & 3.98 & 0.0001 & 0.19 & 0.10 & 0.28 \\
\hline 7 & AURKB & 3.95 & 0.0001 & 0.19 & 0.10 & 0.28 \\
\hline 11 & AURKB & 3.89 & 0.0001 & 0.19 & 0.09 & 0.28 \\
\hline 1 & AURKB & 3.88 & 0.0001 & 0.19 & 0.09 & 0.28 \\
\hline 12 & AURKAPS1 & 3.87 & 0.0001 & 0.19 & 0.09 & 0.28 \\
\hline 9 & WDR12 & 3.87 & 0.0001 & 0.19 & 0.09 & 0.28 \\
\hline 17 & AURKAPS1 & 3.85 & 0.0001 & 0.19 & 0.09 & 0.28 \\
\hline 10 & AURKB & 3.84 & 0.0001 & 0.19 & 0.09 & 0.28 \\
\hline 21 & AURKAPS1 & 3.79 & 0.0002 & 0.18 & 0.09 & 0.28 \\
\hline 4 & AURKA & 3.78 & 0.0002 & 0.18 & 0.09 & 0.28 \\
\hline 22 & WDR12 & 3.77 & 0.0002 & 0.18 & 0.09 & 0.27 \\
\hline 5 & WDR12 & 3.73 & 0.0002 & 0.18 & 0.09 & 0.27 \\
\hline 21 & WDR12 & 3.69 & 0.0002 & 0.18 & 0.08 & 0.27 \\
\hline 15 & AURKA & 3.68 & 0.0003 & 0.18 & 0.08 & 0.27 \\
\hline 16 & AURKB & 3.64 & 0.0003 & 0.18 & 0.08 & 0.27 \\
\hline 20 & AURKB & 3.64 & 0.0003 & 0.18 & 0.08 & 0.27 \\
\hline 12 & AURKA & 3.64 & 0.0003 & 0.18 & 0.08 & 0.27 \\
\hline 4 & WDR12 & 3.63 & 0.0003 & 0.18 & 0.08 & 0.27 \\
\hline 5 & AURKB & 3.62 & 0.0003 & 0.18 & 0.08 & 0.27 \\
\hline 18 & AURKAPS1 & 3.60 & 0.0004 & 0.17 & 0.08 & 0.27 \\
\hline 20 & WDR12 & 3.58 & 0.0004 & 0.17 & 0.08 & 0.27 \\
\hline 21 & AURKB & 3.58 & 0.0004 & 0.17 & 0.08 & 0.27 \\
\hline 9 & AURKB & 3.56 & 0.0004 & 0.17 & 0.08 & 0.27 \\
\hline 17 & BOP1 & 3.50 & 0.0005 & 0.17 & 0.07 & 0.26 \\
\hline 2 & PES1 & 3.49 & 0.0005 & 0.17 & 0.07 & 0.26 \\
\hline 10 & WDR12 & 3.43 & 0.0007 & 0.17 & 0.07 & 0.26 \\
\hline 1 & PES1 & 3.40 & 0.0008 & 0.17 & 0.07 & 0.26 \\
\hline 22 & AURKB & 3.16 & 0.0017 & 0.15 & 0.06 & 0.25 \\
\hline 12 & WDR12 & 3.15 & 0.0018 & 0.15 & 0.06 & 0.25 \\
\hline 18 & AURKB & 3.03 & 0.0026 & 0.15 & 0.05 & 0.24 \\
\hline 17 & PES1 & 2.99 & 0.0030 & 0.15 & 0.05 & 0.24 \\
\hline 14 & AURKB & 2.86 & 0.0044 & 0.14 & 0.04 & 0.23 \\
\hline 1 & AURKAIP1 & 2.82 & 0.0050 & 0.14 & 0.04 & 0.23 \\
\hline 3 & BOP1 & 2.77 & 0.0058 & 0.14 & 0.04 & 0.23 \\
\hline 11 & WDR12 & 2.60 & 0.0097 & 0.13 & 0.03 & 0.22 \\
\hline 8 & PES1 & 2.59 & 0.0100 & 0.13 & 0.03 & 0.22 \\
\hline 19 & AURKAIP1 & 2.58 & 0.0101 & 0.13 & 0.03 & 0.22 \\
\hline 1 & BOP1 & 2.55 & 0.0112 & 0.12 & 0.03 & 0.22 \\
\hline 4 & AURKB & 2.53 & 0.0119 & 0.12 & 0.03 & 0.22 \\
\hline 19 & BOP1 & 2.47 & 0.0138 & 0.12 & 0.02 & 0.22 \\
\hline 14 & PES1 & 2.47 & 0.0139 & 0.12 & 0.02 & 0.22 \\
\hline 19 & PES1 & 2.45 & 0.0149 & 0.12 & 0.02 & 0.21 \\
\hline 3 & PES1 & 2.44 & 0.0152 & 0.12 & 0.02 & 0.21 \\
\hline 5 & PES1 & 2.29 & 0.0225 & 0.11 & 0.02 & 0.21 \\
\hline 22 & PES1 & 2.27 & 0.0235 & 0.11 & 0.02 & 0.21 \\
\hline 13 & PES1 & 2.27 & 0.0236 & 0.11 & 0.02 & 0.21 \\
\hline 15 & PES1 & 2.25 & 0.0249 & 0.11 & 0.01 & 0.20 \\
\hline 13 & BOP1 & 2.25 & 0.0250 & 0.11 & 0.01 & 0.20 \\
\hline 3 & AURKAIP1 & 2.24 & 0.0254 & 0.11 & 0.01 & 0.20 \\
\hline 6 & BOP1 & 2.17 & 0.0307 & 0.11 & 0.01 & 0.20 \\
\hline 15 & AURKB & 2.16 & 0.0314 & 0.11 & 0.01 & 0.20 \\
\hline 21 & PES1 & 2.15 & 0.0321 & 0.11 & 0.01 & 0.20 \\
\hline 17 & AURKAIP1 & 2.13 & 0.0336 & 0.10 & 0.01 & 0.20 \\
\hline 9 & PES1 & 2.13 & 0.0337 & 0.10 & 0.01 & 0.20 \\
\hline 9 & BOP1 & 2.11 & 0.0354 & 0.10 & 0.01 & 0.20 \\
\hline 20 & PES1 & 2.10 & 0.0364 & 0.10 & 0.01 & 0.20 \\
\hline 12 & AURKB & 2.08 & 0.0379 & 0.10 & 0.01 & 0.20 \\
\hline 2 & AURKAIP1 & 2.07 & 0.0389 & 0.10 & 0.01 & 0.20 \\
\hline 21 & AURKAIP1 & 2.05 & 0.0409 & 0.10 & 0.00 & 0.20 \\
\hline 7 & BOP1 & 1.99 & 0.0473 & 0.10 & 0.00 & 0.19 \\
\hline
\end{tabular}


Supplementary Table 11. Univariate analysis for overall survival and recurrence free survival using logrank test for patients from Cohort $\mathbf{D}$.

Overall Survival

\begin{tabular}{lccc}
\hline & \multicolumn{3}{c}{ Univariate analysis } \\
\hline variable & HR & $95 \%$ CI & P-value \\
\hline Age $\geqq 70$ & 3.1 & $1.39-7.59$ & $\mathbf{0 . 0 0 5}$ \\
Male & 0.83 & $0.39-1.84$ & 0.65 \\
Tumor location rectum yes/no & 1.83 & $0.85-4.03$ & 0.12 \\
Tumor size $>45 \mathrm{~mm}$ & 1.04 & $0.48-2.28$ & 0.91 \\
Poorly differentiated histology & 1.53 & $0.45-4.01$ & 0.45 \\
T Stage greater than T4 & 1.07 & $0.43-2.40$ & 0.88 \\
Lymph node metastasis positive & 2.18 & $1.01-5.12$ & $\mathbf{0 . 0 4 9}$ \\
Lymphatic invasion positive & 1.53 & $0.70-3.58$ & 0.3 \\
Venous invasion positive & 0.67 & $0.26-2.30$ & 0.49 \\
UICC stage 3 & 2.18 & $0.99-5.12$ & 0.051 \\
MSI patients yes/no & 1.28 & $0.30-3.69$ & 0.69 \\
CCAT2 high expression & 6.1 & $2.79-13.31$ & $<\mathbf{0 . 0 0 0 1}$ \\
BOP1 high expression & 3.01 & $1.38-6.55$ & $\mathbf{0 . 0 0 6}$ \\
PES1 high expression & 3.52 & $1.62-7.74$ & $\mathbf{0 . 0 0 2}$ \\
WDR12 high expression & 3.15 & $1.44-6.86$ & $\mathbf{0 . 0 0 4}$ \\
\hline
\end{tabular}

Recurrence Free Survival

\begin{tabular}{lccc}
\hline & \multicolumn{3}{c}{ Univariate analysis } \\
\hline variable & HR & $95 \%$ CI & P-value \\
\hline Age $\geqq 70$ & 1.14 & $0.68-1.89$ & 0.62 \\
Male & 0.95 & $0.57-1.61$ & 0.85 \\
Tumor location rectum yes/no & 2.63 & $1.58-4.47$ & $\mathbf{0 . 0 0 0 2}$ \\
Tumor size $>45$ mm & 1.08 & $0.65-1.79$ & 0.77 \\
Poorly differentiated histology & 1.05 & $0.44-2.16$ & 0.9 \\
T Stage greater than T4 & 1.54 & $0.89-2.59$ & 0.12 \\
Lymph node metastasis positive & 1.88 & $1.13-3.18$ & $\mathbf{0 . 0 2}$ \\
Lymphatic invasion positive & 1.38 & $0.83-2.36$ & 0.22 \\
Venous invasion positive & 1.05 & $0.49-2.72$ & 0.92 \\
UICC stage 3 & 1.88 & $1.13-3.18$ & $\mathbf{0 . 0 2}$ \\
MSI patients yes/no & 3.41 & $1.06-20.79$ & $\mathbf{0 . 0 4}$ \\
CCAT2 high expression & 4.78 & $2.74-8.09$ & $<\mathbf{0 . 0 0 0 1}$ \\
BOP1 high expression & 2.77 & $1.65-4.60$ & $\mathbf{0 . 0 0 0 2}$ \\
PES1 high expression & 2.94 & $1.70-4.96$ & $\mathbf{0 . 0 0 0 2}$ \\
WDR12 high expression & 4.07 & $2.18-7.17$ & $<\mathbf{0 . 0 0 0 1}$ \\
\hline
\end{tabular}

HR, Hazard Ratio; CI, Confidence Interval. Bold indicates a statistically significant result. 
Supplementary Table 12. Multivariate analysis for overall survival and recurrence free survival in patients from Cohort D using Cox proportional hazard model.

CCAT2 and other clinical factors

Overall Survival

\begin{tabular}{lcccccc}
\hline & \multicolumn{3}{c}{ Univariate analysis } & \multicolumn{3}{c}{ Multivariate analysis } \\
\hline variable & HR & $95 \%$ CI & P-value & HR & $95 \%$ CI & P-value \\
\hline Age $\geqq 70$ & 3.1 & $1.39-7.59$ & $\mathbf{0 . 0 0 5}$ & 2.41 & $1.05-6.01$ & $\mathbf{0 . 0 4}$ \\
Lymph node metastasis & 2.18 & $1.01-5.12$ & $\mathbf{0 . 0 4 9}$ & 2.42 & $1.10-5.69$ & $\mathbf{0 . 0 3}$ \\
positive & 6.1 & $2.79-13.31$ & $<\mathbf{0 . 0 0 0 1}$ & 5.51 & $2.48-12.27$ & $<\mathbf{0 . 0 0 0 1}$ \\
CCAT2 high expression & \multicolumn{7}{c}{} & & \\
\hline Recurrence Free Survival & \multicolumn{7}{c}{ Univariate analysis } & Multivariate analysis \\
\hline & HR & $95 \%$ CI & P-value & HR & $95 \%$ CI & P-value \\
\hline variable & 2.63 & $1.58-4.47$ & $\mathbf{0 . 0 0 0 2}$ & 2.37 & $1.41-4.08$ & $\mathbf{0 . 0 0 1}$ \\
Tumor location rectum yes/no & 1.88 & $1.13-3.18$ & $\mathbf{0 . 0 2}$ & 2.03 & $1.21-3.45$ & $\mathbf{0 . 0 0 7}$ \\
Lymph node metastasis & 1.88 & $1.13-3.18$ & $\mathbf{0 . 0 2}$ & & & \\
positive & 3.41 & $1.06-20.79$ & $\mathbf{0 . 0 4}$ & 0.5 & $0.08-1.66$ & 0.29 \\
UICC stage 3 & 4.78 & $2.74-8.09$ & $<\mathbf{0 . 0 0 0 1}$ & 4.85 & $2.76-8.28$ & $<\mathbf{0 . 0 0 0 1}$ \\
MSI patients yes/no & CCAT2 high expression &
\end{tabular}

HR, Hazard Ratio; CI, Confidence Interval. Bold indicates a statistically significant result.

$B O P 1$ and other clinical factors

Overall Survival

\begin{tabular}{lcccccc}
\hline & \multicolumn{3}{c}{ Univariate analysis } & \multicolumn{3}{c}{ Multivariate analysis } \\
\hline variable & HR & $95 \%$ CI & P-value & HR & $95 \%$ CI & P-value \\
\hline Age $\geqq 70$ & 3.1 & $1.39-7.59$ & $\mathbf{0 . 0 0 5}$ & 3.06 & $1.36-7.49$ & $\mathbf{0 . 0 0 6}$ \\
Lymph node metastasis & 2.18 & $1.01-5.12$ & $\mathbf{0 . 0 4 9}$ & 2.6 & $1.18-6.12$ & $\mathbf{0 . 0 2}$ \\
positive & 3.01 & $1.38-6.55$ & $\mathbf{0 . 0 0 6}$ & 3.12 & $1.42-6.84$ & $\mathbf{0 . 0 0 5}$ \\
BOP1 high expression & \multicolumn{7}{c}{ Multivariate analysis } \\
\hline Recurrence Free Survival & \multicolumn{7}{c}{ Univariate analysis } & HR & $95 \%$ CI & P-value \\
\hline & HR & $95 \% C I$ & P-value & H. & \\
\hline variable & 2.63 & $1.58-4.47$ & $\mathbf{0 . 0 0 0 2}$ & 2.38 & $1.42-4.09$ & $\mathbf{0 . 0 0 1}$ \\
\hline Tumor location rectum yes/no & 1.88 & $1.13-3.18$ & $\mathbf{0 . 0 2}$ & 1.87 & $1.12-3.18$ & $\mathbf{0 . 0 2}$ \\
Lymph node metastasis & 1.88 & $1.13-3.18$ & $\mathbf{0 . 0 2}$ & & & \\
positive & 3.41 & $1.06-20.79$ & $\mathbf{0 . 0 4}$ & 0.52 & $0.08-1.73$ & 0.32 \\
UICC stage 3 & 2.77 & $1.65-4.60$ & $\mathbf{0 . 0 0 0 2}$ & 2.67 & $1.59-4.45$ & $\mathbf{0 . 0 0 0 3}$ \\
MSI patients yes/no & BOP1 high expression & &
\end{tabular}

HR, Hazard Ratio; CI, Confidence Interval. Bold indicates a statistically significant result. 


\section{References}

1. Morikawa K, Walker SM, Nakajima M, et al. Influence of organ environment on the growth, selection, and metastasis of human colon carcinoma cells in nude mice. Cancer Res 1988;48:6863-71.

2. Shah MY, Ferracin M, Pileczki V, et al. Cancer-associated rs6983267 SNP and its accompanying long noncoding RNA CCAT2 induce myeloid malignancies via unique SNP-specific RNA mutations. Genome Res 2018;28:432-447.

3. Lee SM, Kim N, Son HJ, et al. The Effect of Sex on the Azoxymethane/Dextran Sulfate Sodium-treated Mice Model of Colon Cancer. J Cancer Prev 2016;21:271-278.

4. Parang B, Barrett CW, Williams CS. AOM/DSS Model of Colitis-Associated Cancer. Methods Mol Biol 2016;1422:297-307.

5. Suzuki R, Kohno H, Sugie $\mathrm{S}$, et al. Strain differences in the susceptibility to azoxymethane and dextran sodium sulfate-induced colon carcinogenesis in mice. Carcinogenesis 2006;27:162-9.

6. Gerling M, Glauben R, Habermann JK, et al. Characterization of chromosomal instability in murine colitisassociated colorectal cancer. PLoS One 2011;6:e22114.

7. Sato T, Vries RG, Snippert HJ, et al. Single Lgr5 stem cells build crypt-villus structures in vitro without a mesenchymal niche. Nature 2009;459:262-5.

8. Sato $\mathrm{T}$, Stange $\mathrm{DE}$, Ferrante $\mathrm{M}$, et al. Long-term expansion of epithelial organoids from human colon, adenoma, adenocarcinoma, and Barrett's epithelium. Gastroenterology 2011;141:1762-72.

9. Miyoshi $\mathrm{H}$, Stappenbeck TS. In vitro expansion and genetic modification of gastrointestinal stem cells in spheroid culture. Nat Protoc 2013;8:2471-82.

10. Song S, Wang Z, Li Y, et al. PPARdelta Interacts with the Hippo Coactivator YAP1 to Promote SOX9 Expression and Gastric Cancer Progression. Mol Cancer Res 2020;18:390-402.

11. Katsiampoura A, Raghav K, Jiang ZQ, et al. Modeling of Patient-Derived Xenografts in Colorectal Cancer. Mol Cancer Ther 2017;16:1435-1442.

12. Liu J, Lichtenberg T, Hoadley KA, et al. An Integrated TCGA Pan-Cancer Clinical Data Resource to Drive High-Quality Survival Outcome Analytics. Cell 2018;173:400-416 e11.

13. Cancer Genome Atlas N. Comprehensive molecular characterization of human colon and rectal cancer. Nature 2012;487:330-7.

14. Redis RS, Vela LE, Lu W, et al. Allele-Specific Reprogramming of Cancer Metabolism by the Long Noncoding RNA CCAT2. Mol Cell 2016;61:520-534.

15. Song L, Bhuvaneshwar K, Wang Y, et al. CINdex: A Bioconductor Package for Analysis of Chromosome Instability in DNA Copy Number Data. Cancer Inform 2017;16:1176935117746637.

16. Ling $\mathrm{H}$, Spizzo R, Atlasi $\mathrm{Y}$, et al. CCAT2, a novel noncoding RNA mapping to 8q24, underlies metastatic progression and chromosomal instability in colon cancer. Genome Res 2013;23:1446-61.

17. Gagnon KT, Li L, Janowski BA, et al. Analysis of nuclear RNA interference in human cells by subcellular fractionation and Argonaute loading. Nat Protoc 2014;9:2045-60.

18. Reuter JS, Mathews DH. RNAstructure: software for RNA secondary structure prediction and analysis. BMC Bioinformatics 2010;11:129.

19. Das R, Laederach A, Pearlman SM, et al. SAFA: semi-automated footprinting analysis software for highthroughput quantification of nucleic acid footprinting experiments. RNA 2005;11:344-54.

20. Deigan KE, Li TW, Mathews DH, et al. Accurate SHAPE-directed RNA structure determination. Proc Natl Acad Sci U S A 2009;106:97-102.

21. Zhou P. Determining protein half-lives. Methods Mol Biol 2004;284:67-77.

22. Pereboom TC, Bondt A, Pallaki $P$, et al. Translation of branched-chain aminotransferase- 1 transcripts is impaired in cells haploinsufficient for ribosomal protein genes. Experimental Hematology 2014;42:394403.

23. Reineke LC, Cheema SA, Dubrulle J, et al. Chronic starvation induces noncanonical pro-death stress granules. J Cell Sci 2018;131.

24. Dassi E. Post-Transcriptional Gene Regulation: New York: Springer Science and Business, 2016. 
25. Ahmed D, Eide PW, Eilertsen IA, et al. Epigenetic and genetic features of 24 colon cancer cell lines. Oncogenesis 2013;2:e71.

26. Knutsen T, Padilla-Nash HM, Wangsa D, et al. Definitive molecular cytogenetic characterization of 15 colorectal cancer cell lines. Genes Chromosomes Cancer 2010;49:204-23.

27. Camps J, Morales C, Prat E, et al. Genetic evolution in colon cancer KM12 cells and metastatic derivates. Int J Cancer 2004;110:869-74.

28. Leroy B, Girard L, Hollestelle A, et al. Analysis of TP53 mutation status in human cancer cell lines: a reassessment. Hum Mutat 2014;35:756-65.

29. Quinn LA, Moore GE, Morgan RT, et al. Cell lines from human colon carcinoma with unusual cell products, double minutes, and homogeneously staining regions. Cancer Res 1979;39:4914-24.

30. Chen TR, Drabkowski D, Hay RJ, et al. WiDr is a derivative of another colon adenocarcinoma cell line, HT29. Cancer Genet Cytogenet 1987;27:125-34.

31. Yao Y, Tao H, Kim JJ, et al. Alterations of DNA mismatch repair proteins and microsatellite instability levels in gastric cancer cell lines. Lab Invest 2004;84:915-22.

32. Barranco SC, Townsend CM, Jr., Casartelli C, et al. Establishment and characterization of an in vitro model system for human adenocarcinoma of the stomach. Cancer Res 1983;43:1703-9.

33. Cheng LL, Itahana Y, Lei ZD, et al. TP53 genomic status regulates sensitivity of gastric cancer cells to the histone methylation inhibitor 3-deazaneplanocin A (DZNep). Clin Cancer Res 2012;18:4201-12.

34. Sekiguchi M, Sakakibara K, Fujii G. Establishment of cultured cell lines derived from a human gastric carcinoma. Jpn J Exp Med 1978;48:61-8.

35. Yokozaki H. Molecular characteristics of eight gastric cancer cell lines established in Japan. Pathol Int 2000;50:767-77. 